\title{
The history of degenerate (bipartite) extremal graph problems
}

\author{
Zoltán Füredi and Miklós Simonovits \\ May 15, 2013 \\ Alfréd Rényi Institute of Mathematics, Budapest, Hungary \\ z-furedi@illinois.edu and \\ simonovits.miklos@renyi.mta.hu
}

\begin{abstract}
This paper is a survey on Extremal Graph Theory, primarily focusing on the case when one of the excluded graphs is bipartite. On one hand we give an introduction to this field and also describe many important results, methods, problems, and constructions. 1
\end{abstract}

\section{Contents}

1 Introduction 4

1.1 Some central theorems of the field . . . . . . . . . 5

1.2 The structure of this paper . . . . . . . . . . . 6

1.3 Extremal problems . . . . . . . . . . . . . 8

1.4 Other types of extremal graph problems . . . . . . . . . . 10

1.5 Historical remarks . . . . . . . . . . . . . . . 11

2 The general theory, classification 12

2.1 The importance of the Degenerate Case . . . . . . . . . . 14

2.2 The asymmetric case of Excluded Bipartite graphs . . . . . 15

2.3 Reductions: Host graphs . . . . . . . . . . . . . . . 16

2.4 Excluding complete bipartite graphs . . . . . . . . . . 17

2.5 Probabilistic lower bound . . . . . . . . . . . 18

${ }^{1}$ Research supported in part by the Hungarian National Science Foundation OTKA 104343, and by the European Research Council Advanced Investigators Grant 267195 (ZF) and by the Hungarian National Science Foundation OTKA 101536, and by the European Research Council Advanced Investigators Grant 321104. (MS). 
Füredi-Simonovits: Degenerate (bipartite) extremal graph problems

2.6 Classification of extremal problems . . . . . . . . . 21

2.7 General conjectures on bipartite graphs . . . . . . . . . . 23

3 Excluding complete bipartite graphs 24

3.1 Bipartite $C_{4}$-free graphs and the Zarankiewicz problem . . . . 24

3.2 Finite Geometries and the $C_{4}$-free graphs . . . . . . . . 26

3.3 Excluding $C_{4}$ : Exact results . . . . . . . . . . . 28

3.4 Excluding $K(2, t+1), t>1 \ldots \ldots \ldots \ldots \ldots$

3.5 Excluding $K(3,3)$, and improving the upper bound . . . . . 32

3.6 Further applications of Algebraic Methods . . . . . . . . . 34

3.7 The coefficient in the Kővári-T. Sós-Turán bound . . . . . . . 37

3.8 Excluding large complete subgraphs . . . . . . . . . 37

4 Excluding Cycles : $C_{2 k}$

4.1 Girth and Turán numbers, upper bounds . . . . . . . . . 38

4.2 Excluding a single $C_{2 k}$, upper bounds . . . . . . . . . . 40

4.3 Eliminating short cycles, a promising attempt . . . . . . . . 42

4.4 A lower bound for $C_{6}$ : The Benson Construction . . . . . . . 43

4.5 Girth 12 graphs by Benson and by Wenger . . . . . . . . . . 44

4.6 Short cycles, $C_{6}$ and $C_{10} \ldots \ldots \ldots \ldots \ldots$

4.7 Bipartite hosts with extreme sides . . . . . . . . . 47

4.8 The effect of odd cycles . . . . . . . . . . . . . 47

4.9 Large girth: Ramanujan graphs . . . . . . . . . . . . . . . . . 49

4.10 The girth problem: the Lazebnik-Ustimenko approach . . . . 52

4.11 Cycle length distribution . . . . . . . . . . . . 53

5 Paths and long cycles 54

5.1 Excluding long cycles . . . . . . . . . . . . . . . . . . . . . . . . . 54

$5.2 \quad$ Excluding $P_{k} \ldots \ldots \ldots \ldots \ldots \ldots \ldots$

5.3 Proof ideas . . . . . . . . . . . . . . . 57

5.4 Generalizations . . . . . . . . . . . . . . 5 57

6 Excluding trees 58

6.1 Erdös-Sós conjecture . . . . . . . . . . . . . . . . 58

6.2 Sketch of the proof of Theorem $6.2 \ldots \ldots \ldots$. . . . . . 59

6.3 Komlós-Sós conjecture on median degree . . . . . . . . . . . 60

7 More complex excluded subgraphs 61

7.1 The Erdös-Simonovits Reduction and the Cube theorem . . 61

7.2 Theta graphs and the Faudree-Simonovits reduction . . . . 64 
7.3 A universal graph and dependent random choice . . . . . 65

8 Eigenvalues and extremal problems 68

9 Excluding topological subdivisions $\quad 69$

9.1 Large topological subgraphs . . . . . . . . . . . . . . . 69

9.2 Turán numbers of subdivided graphs . . . . . . . . . . . 70

10 Hypergraph Extremal Problems $\quad 70$

10.1 Positive Density problems . . . . . . . . . . . . . 70

10.2 Degenerate hypergraph problems . . . . . . . . . 71

11 Supersaturated graphs $\quad 72$

11.1 Erdős-Simonovits-Sidorenko conjecture . . . . . . . . . 73

12 Ordered structures

12.1 Directed graphs, ordered graphs . . . . . . . . . . 75

12.2 Erdös-Moser conjecture on unit distances . . . . . . . . . . 75

12.3 Ordered submatrices . . . . . . . . . . . . . 76

12.4 Ordered matrices and the Stanley-Wilf conjecture on subper-

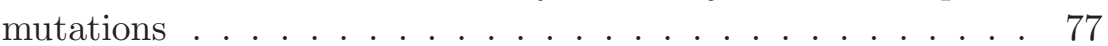

13 Applications in Geometry 78

13.1 Applicability of the Kővári-T. Sós-Turán bound . . . . . . . 78

13.2 Unit Distances . . . . . . . . . . . . . . . . 78

13.3 Cells in line arrangements . . . . . . . . . . . . . . 79

14 Further connections and problems 8

14.1 Connections of hypergraphs and critical graphs . . . . . . . 80

14.2 A multiplicative Sidon problem and $C_{2 k}$-free graphs $\ldots . . .83$

14.3 Cycle-free subgraphs of the $d$-dimensional hypercube . . . . . 83

14.4 Two problems of Erdös . . . . . . . . . . . . . . . 84

15 Acknowledgements 84 


\section{Introduction}

This survey describes the theory of Degenerate Extremal Graph Problems, the main results of the field, and its connection to the surrounding areas.

Extremal graph problems we consider here are often called Turán type extremal problems, because Turán's theorem and his questions were the most important roots of this huge area [242], 243].

Generally, we have a Universe of graphs, $\mathbb{U}$, where this universe may be the family of ordinary graphs, or digraphs, or hypergraphs, or ordered graphs, or bipartite graphs, etc and a property $\mathcal{P}$, saying, e.g., that $G \in \mathbb{U}$ does not contain some subgraphs $L \in \mathcal{L}$, or that it is Hamiltonian, or it is at most 3 -chromatic, and we have some parameters on $\mathbb{U}$, say $v(G)$ and $e(G)$, the number of vertices and edges. Our aim is to maximize the second parameter under the condition that $G$ has property $\mathcal{P}$ and its first parameter is given.

We call such a problem Turán type extremal problem if we are given a family $\mathcal{L}$ of graphs from our universe, $G_{n}$ is a graph of $n$ vertices, $e\left(G_{n}\right)$ denotes the number of edges of $G_{n}$ and we try to maximize $e\left(G_{n}\right)$ under the condition that $G_{n}$ contains no $L \in \mathcal{L}$, where "contains" means "not necessarily induced subgraph". (Here graph may equally mean digraph, or multigraph, or hypergraph).

The maximum will be denoted by $\operatorname{ex}(n, \mathcal{L})$ and the graphs attaining this maximum without containing subgraphs from $\mathcal{L}$ are called extremal graphs. The family of extremal graphs is denoted by $\mathbf{E X}(n, \mathcal{L})$ and $\mathbf{e x}(n, \mathcal{L})$ is called the Turán number of the family $\mathcal{L}$.

Speaking of $\operatorname{ex}(n, L)$ we shall always assume that $n \geq|V(L)|$, otherwise the problem is trivial.

Definition 1.1. If the Universe $\mathbb{U}$ is the family of $r$-uniform hypergraph 2 , then we shall call the problem degenerate if the maximum,

$$
\operatorname{ex}(n, \mathcal{L})=o\left(n^{r}\right) .
$$

Otherwise we shall call it non-degenerate

Below we shall mention several open problems. Yet to get more problems, we refer the reader to the

\footnotetext{
${ }^{2} r=2$ included, moreover, mostly we think of $r=2$.
} 


\section{Erdős homepage: www.renyi.hu/ $\quad$ p_erdos}

where the papers of Erdös can be found [59]. Also, many open problems can be found in Chung-Graham [47.

\subsection{Some central theorems of the field}

We start with some typical theorems of the field and two conjectures. The aim of this "fast introduction" is to give a feeling for what are the crucial types of results here.

Theorem 1.2 (Kövári-T. Sós-Turán, [164]). Let $K_{a, b}$ denote the complete bipartite graph with $a$ and $b$ vertices in its color-classes. Then

$$
\operatorname{ex}\left(n, K_{a, b}\right) \leq \frac{1}{2} \sqrt[a]{b-1} \cdot n^{2-(1 / a)}+O(n)
$$

We use this theorem with $a \leq b$, since that way we get a better estimate.

Theorem 1.3 (Kollár-Alon-Rónyai-Szabó [159], [11]). If $b>(a-1)$ !, then

$$
\operatorname{ex}\left(n, K_{a, b}\right)>c_{a} n^{2-(1 / a)}
$$

Theorem 1.4 (Erdős, Bondy and Simonovits [32]).

$$
\operatorname{ex}\left(n, C_{2 k}\right) \leq 100 k n^{1+(1 / k)} .
$$

Theorem 1.5 (Erdős-Simonovits, Cube Theorem [90]). Let $Q_{8}$ denote the cube graph defined by the vertices and edges of a 3-dimensional cube. Then

$$
\operatorname{ex}\left(n, Q_{8}\right)=O\left(n^{8 / 5}\right)
$$

Conjecture 1.6 (Erdős and Simonovits, Rational exponents). For any finite family $\mathcal{L}$ of graphs, if there is a bipartite $L \in \mathcal{L}$, then there exists a rational $\alpha \in[0,1)$ and a $c>0$ such that

$$
\frac{\operatorname{ex}(n, \mathcal{L})}{n^{1+\alpha}} \rightarrow c .
$$

Theorem 1.7 (Füredi [11], [104]). If $q \neq 1,7,9,11,13$, and $n=q^{2}+q+1$, then

$$
\operatorname{ex}\left(n, C_{4}\right) \leq \frac{1}{2} q(q+1)^{2}
$$

Moreover, if $q$ is a power of a prime, then

$$
\operatorname{ex}\left(n, C_{4}\right)=\frac{1}{2} q(q+1)^{2} .
$$


Conjecture 1.8 (Erdős). 3

$$
\operatorname{ex}\left(n,\left\{C_{3}, C_{4}\right\}\right)=\frac{1}{2 \sqrt{2}} n^{3 / 2}+o\left(n^{3 / 2}\right) .
$$

We close this part with a famous result of Ruzsa and Szemerédi:

Theorem 1.9 (Solution of the (6,3) problem, [210]). If $\mathcal{H}_{n}^{(3)}$ is a 3-uniform hypergraph not containing 6 vertices determining (at least) 3 hyperedges, then this hypergraph has o( $\left.n^{2}\right)$ hyperedges.

The above theorems will be discussed in more details below.

\subsection{The structure of this paper}

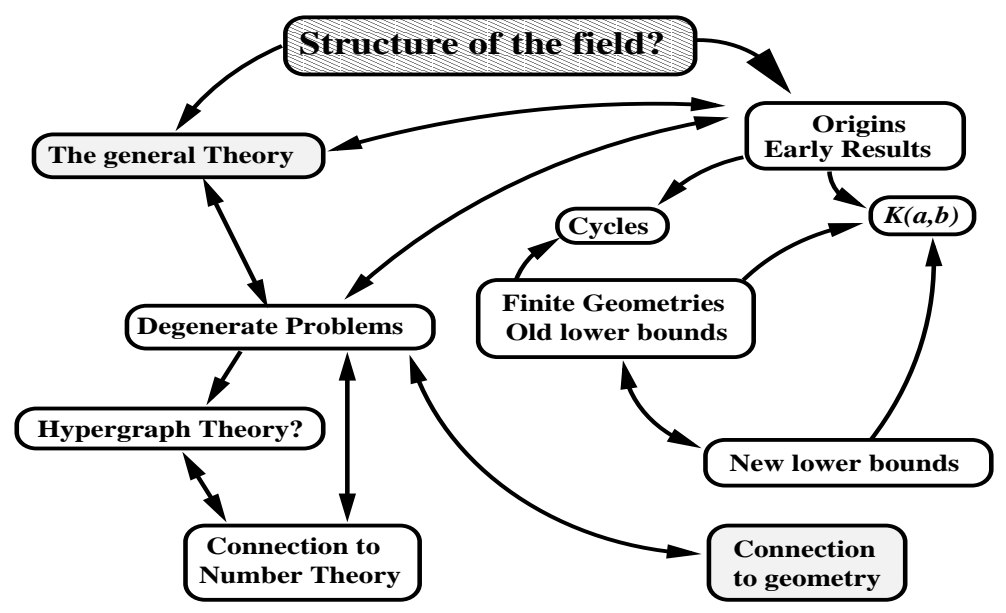

Figure 1: Area Map

The area is fairly involved. Figure 1 shows a complicated - but not complete - map of the interactions of some subfields of the field discussed here. We start with describing the Extremal problems in general, then move to the Degenerate problems, also describing why they are important. Among the most important degenerate extremal problems we mention are the extremal problem of $K_{a, b}$, and also $C_{2 k}$, where - to classify the extremal problems - we shall need the Random Graph Method to get a lower bound in the problem of $C_{2 k}$. These results are enough to give a good classification of degenerate extremal graph problems.

\footnotetext{
${ }^{3}$ This conjecture is mentioned in [70] but it is definitely older, see e.g. Brown, [37] .
} 
1. The two lowest boxes of Figure 1 show that this whole area has a strong connection to Geometry and Number Theory. This will be explained in Sections 13, 1.5, and 14.2,

2. The origins of this field are

(a) an early, singular result of Mantel,

(b) the multiplicative Sidon Problem (see Section 1.5)

(c) Turán's theorem and his systematic approach to the field.

So the origins come - in some sense - from Number Theory, are strongly connected to Finite Geometry, and in this way also to ordinary geometry (Turán's theorem comes from the Erdős-Szekeres version of Ramsey Theorem, which they invented to solve the Esther Klein problem from Geometry.)

3. To understand the field we start with a very short description of the general theory, and then - skipping most of the hypergraph theory - we move to the main area of this paper: to the questions where we consider ordinary extremal graphs, and exclude some bipartite $L$ : therefore, by Theorem 1.2, we have $\operatorname{ex}(n, \mathcal{L})=O\left(n^{2-c}\right)$.

4. One important phenomenon is that many extremal graph problems can be "reduced" to some degenerate extremal graph problems that we also call sometimes bipartite extremal problems.

5. The upper bounds in the simpler cases are obtained by some double counting, Jensen type inequalities, or applying some supersaturated graph theorems 4

6. There are also much more complicated cases, where the above simple approach is not enough, we need some finer arguments. Perhaps the first such case was treated by Füredi: Section 7.3 and [107. Also such an approach is the application of the general Dependent Random Choice Method, (see the survey of Fox and Sudakov [101]).

7. The lower bounds are sometimes provided by random graphs (see Section (2.5) but these are often too weak. So we often use some finite geometric constructions, (see Section 3.2) or their generalizations coming from commutative algebras (see Sections 3.6, 4.9, 8), etc., and they occasionally provide matching upper and lower bounds. Again, there is an important general method with many important results,

\footnotetext{
${ }^{4}$ Lovász and Szegedy had a beautiful conjecture, which we formulate here only in a restricted form: Any (valid) extremal theorem can be proven by applying the CauchySchwarz inequality finitely many times. This conjecture was killed in this strong form by Hatami and Norine 142 - but proven in a weaker, "approximation-form".
} 
which we shall call the Lazebnik-Ustimenko method but will treat only very superficially in Section 3.6.

\subsection{Extremal problems}

We shall almost entirely restrict ourselves to Turán type extremal problems for ordinary simple graphs, i.e. loops, multiple edges are excluded.

To show the relation of the areas described here, we start with a list of some related areas.

1. Ramsey Theory

- Problems not connected to density problems; in some sense these are the real Ramsey Problems

- Problems connected to density problems, i.e. cases, where we do not really use the Ramsey Condition, only that some color class is large.

2. Ordinary extremal graph theory

- Excluding bipartite graphs (degenerate problems)

- Excluding topological subgraphs (very degenerate extremal problems)

- Matrix problems, ordered and not ordered;

- Non-degenerate case, and its relation to degenerate problems

3. Theory of extremal digraph problems

4. Ramsey-Turán Problems

5. Connection to Random Graphs

6. Hypergraph extremal problems

7. Connection of Number Theoretical problems to Extremal Graph Theory

8. Continuous problems

9. Applications

There are several surveys on these fields, see e.g., T. Sós [232], Füredi [108], [110], Simonovits [224], [228], 227], 222], Simonovits and Sós [230], [155]. Perhaps the first survey on this topic was Vera Sós' paper [232], discussing connections between extremal graph problems, finite geometries, block designs, etc. and, perhaps, the nearest to this survey is [225], Bollobás, [28] Sidorenko [217], [101], and also some books, e.g., Bollobás [26]. Of 
course, a lot of information is hidden in the papers of Erdős, among others, in [70], [73], 76].

So, here we shall concentrate on Case 2, but to position this area we shall start with some related fields, among others, with the general asymptotic in Case 2.

Problem 1.10 (General Host-graphs). In a more general setting we have a sequence $\left(H_{n}\right)$ of "host" graphs and the question is, how many edges can a subgraph $G_{n} \subset H_{n}$ have under the condition that it does not contain any forbidden subgraph $L \in \mathcal{L}$. The maximum will be denoted by $\operatorname{ex}\left(H_{n}, \mathcal{L}\right)$.

For $H_{n}=K_{n}$ we get back the ordinary extremal graph problems. There are several further important subcases of this question:

(a) when $H_{n}=K_{a, b}$ for $a \approx n / 2$;

(b) when the host-graph is the $d$-dimensional cube, $n=2^{d}$; see Section 14.3 .

(c) when $H_{n}$ is a random graph on $n$ vertices, see e.g. 209].

Notation. Given some graphs $G_{n}, T_{n, p}, T_{k}, H_{\nu}, \ldots$ the (first) subscript will almost always denote the number of vertices 5 So $K_{p}$ is the complete graph on $p$ vertices, $P_{k}$ the path on $k$ vertices, $C_{k}$ is the cycle of $k$ vertices, while $\mathcal{C}_{\geq k}$ will denote the family of cycles of length at least $k . \delta(x)$ denotes the degree of the vertex $x$.

The complete bipartite graph $K_{a, b}$ with $a$ vertices in its first class and $b$ in its 2 nd class will be crucial in this paper. Often, we shall denote it by $K(a, b)$, and its $p$-partite generalization by $K_{p}\left(a_{1}, \ldots, a_{p}\right)$. If $\sum a_{i}=n$ and $\left|n_{i}-n_{j}\right| \leq 1$, then $K_{p}\left(a_{1}, \ldots, a_{p}\right)$ is the Turán graph $T_{n, p}$ on $n$ vertices and $p$ classes.

Given two graphs $U$ and $W$, their product graph is the one obtained from vertex-disjoint copies of these two graphs by joining each vertex of $U$ to each vertex of $W$. This will be denoted by $U \otimes W 6$

Given a graph $H, v(H)$ is its number of vertices, $e(H)$ its number of edges and $\chi(H)$ its chromatic number, $d_{\min }(G)$ and $d_{\max }(G)$ denote the minimum and maximum degrees of $G$, respectively.

We shall write $f(x) \approx g(x)$ if $f(x) / g(x) \rightarrow 1$. Occasionally [n] denote the set of first $n$ integers, $[n]:=\{1,2, \ldots, n\}$.

The Overlap. Some twenty years ago Simonovits wrote a survey 227] on the influence of Paul Erdős in the areas described above, Many-many

\footnotetext{
${ }^{5}$ One important exception is the complete bipartite graph $K(a, b)=K_{a, b}$, see below. Another exception is, when we list some excluded subgraphs, like $L_{1}, \ldots, L_{\nu}$.

${ }^{6}$ This product is often called also the joint of the two graphs.
} 
features of these areas changed drastically since that. Jarik Nešetřil and Ron Graham were the editors of that survey-volume, and now they decided to republish it. Fortunately, the authors had the option to slightly rewrite their original papers. Simonovits has rewritten his original paper [228], basically keeping everything he could but indicating many new developments, and adding remarks and many new references to it.

To make this paper readable and self-contained, we shall touch on some basic areas also treated there, or in other survey papers of ours, Here, however, we shall explain many-many results and phenomena just mentioned in other survey papers.

Remark 1.11. There is also a third new survey to be mentioned here: Simonovits gave a lecture at the conference on Turán's $100^{\text {th }}$ anniversary, in 2011. His lecture tried to cover the whole influence of Paul Turán in Discrete Mathematics. In the volume of this conference Simonovits wrote a survey 229] covering his lecture, except that

- the area called Statistical Group Theory is discussed in a survey of Pálfy and Szalay [200] and

- some parts of the applications of Extremal Graph Theory, primarily in Probability Theory are covered by Katona [153], in the same volume.

\subsection{Other types of extremal graph problems}

Above we still tried to maximize the number of edges, hyperedges, etc. More generally, instead of maximizing $e\left(G_{n}\right)$, we may maximize something else:

1. Min-degree problems (or Dirac type problems): How large min-degree can $G_{n}$ have without containing subgraphs from $\mathcal{L}$.

2. Median problems which will be called here Loebl-Komlós-Sós type problems: Given a graph $G_{n}$, which $m$ and $d$ ensure that if $G_{n}$ has at least $m$ vertices of degree $\geq d$, then $G_{n}$ contains some $L \in \mathcal{L}$.

3. Eigenvalue-extremal problems 7: maximize the maximum eigenvalue $\lambda\left(G_{n}\right)$ under the condition that $G_{n}$ does not contain any $L \in \mathcal{L}$. (These are sharper forms of some extremal results, since the maximum eigenvalue

$$
\lambda\left(G_{n}\right) \geq \frac{2 e\left(G_{n}\right)}{n}
$$

see Section 8.)

\footnotetext{
${ }^{7}$ As usual, given a graph $G_{n}$, an $n \times n$ matrix is associated to it, having $n$ eigenvalues. The largest and the second largest is what we are mostly interested in.
} 
4. Subgraph count inequalities, which assert that if $G_{n}$ contains many copies of some subgraphs $L_{1}, \ldots, L_{\lambda}$, then we have at least one (or maybe "many") subgraphs $L$.

5. Diameter-extremal problems. Here we mention just a subcase: if

$$
\operatorname{diam}\left(G_{n}\right) \leq d \quad \text { and } \quad d_{\max }\left(G_{n}\right)<M,
$$

at least how many edges must $G_{n}$ have. The Erdös-Rényi paper [85] is of importance here and also some related papers, like Füredi [105, [109.

6. Combined extremal problems: There are many-many further types of extremal problems. Here we mention, as an example, the results of Balister, Bollobás, Riordan and Schelp [17, where an odd cycle is excluded, and at the same time an upper bound is fixed on the degrees and the number of edges are to be maximized.

The approach 4 is very popular in the theory of Graph Limits [177]. We mention a breakthrough in this area in connection with Erdös' combinatorial problems, of type 4. A famous conjecture of Erdős was

Conjecture 1.12 (Erdös [74]). A $K_{3}$-free $G_{n}$ contains at most $\left(\frac{n}{5}\right)^{5}$ copies of $C_{5}$ 's.

The motivation of this is that the blownup $8 C_{5}$, i.e. $C_{5}[n / 5]$ has no triangles and has $\left(\frac{n}{5}\right)^{5}$ copies of $C_{5}$. Erdös conjectured that no triangle-free $G_{n}$ can have more $C_{5}$ 's than this. The first "approximation" was due to Ervin Győri:

Theorem 1.13 (Györi [133]). A $K_{3}$-free $G_{n}$ contains at most $1.03\left(\frac{n}{5}\right)^{5} C_{5}$ 's

Next Füredi improved the constant to 1.001 (unpublished) [114], and finally independently Grzesik [124], and Hatami, Hladký, Král, Norine, and Razborov [141] proved the conjecture.

\subsection{Historical remarks}

Erdős in 1938 [60] considered the following "multiplicative Sidon Problem"9].

\footnotetext{
${ }^{8}$ Given a graph $H$, its blownup version $H[t]$ is defined as follows: we replace each vertex $x$ of $H$ by $t$ independent new vertices and we join two new vertices coming from distinct vertices $x, y$ iff $x y$ was an edge of $H$.

${ }^{9}$ For a longer description of the number theoretical parts see 228. Erdös also refers to his "blindness" overlooking the general problem in 70 .
} 
Problem 1.14. How many integers, $a_{1}, \ldots, a_{m} \in[1, n]$ can we find so that $a_{i} a_{j}=a_{k} a_{\ell}$ does not hold for any $i, j, k, \ell$, unless $\{i, j\}=\{k, \ell\}$.

To get an upper bound in Problem 1.14, Erdős proved

Theorem 1.15. Let $G[n, n]$ be a bipartite graph with $n$ vertices in both classes. If it does not contain $C_{4}$, then $e(G[n, n])<3 n \sqrt{n}$,

Much later this problem was asked in a more general setting: find an upper bound on $e(G[n, n])$ if $K_{a, b} \not \subset G[n, n]$. Zarankiewicz [254] posed the following question:

Problem 1.16 (Zarankiewicz problem). Determine the largest integer $Z(m, n, a, b)$ for which there is an $m \times n 0-1$ matrix containing $Z(m, n, a, b) 1$ 's without an $a \times b$ submatrix consisting entirely of 1 's.

Hartman, Mycielski and Ryll-Nardzevski [139] gave upper and lower bounds for the case $a=b=2$, weaker than the Erdös-Klein 10 result, and Kővári, T. Sós and Turán (see Theorem 1.2) provided a more general upper bound. We shall discuss these problems and results in details in Sections 2.4 and 3.2 ,

While exact values of $Z(m, n, a, b)$ are known for infinitely many parameter values, mostly only asymptotic bounds are known in the general case. Even $Z(m, n, 2,2)$ is not sufficiently well known.

\section{The general theory, classification}

In many ordinary extremal problems the minimum chromatic number plays a decisive role. The subchromatic number $p(\mathcal{L})$ of $\mathcal{L}$ is defined by

$$
p(\mathcal{L})=\min \{\chi(L): L \in \mathcal{L}\}-1 .
$$

Recall that the Turán graph $T_{n, p}$ is the largest graph on $n$ vertices and $p$ classes.

\section{Claim 2.1.}

$$
\operatorname{ex}(n, \mathcal{L}) \geq e\left(T_{n, p}\right)=\left(1-\frac{1}{p}\right)\left(\begin{array}{l}
n \\
2
\end{array}\right)+o\left(n^{2}\right)
$$

\footnotetext{
${ }^{10}$ In [70] Erdős (again) attributes the finite geometric construction to Eszter (Esther) Klein.
} 
Indeed, $T_{n, p}$ does not contain any $L \in \mathcal{L}$. An easy consequence of the Erdős-Stone theorem [95] provides the asymptotic value of $\mathbf{e x}(n, \mathcal{L})$, at least if $p(\mathcal{L})>1$.

Theorem 2.2 (Erdös-Simonovits [89]). If $\mathcal{L}$ is a family of graphs with subchromatic number $p>0$, then

$$
\operatorname{ex}(n, \mathcal{L})=\left(1-\frac{1}{p}\right)\left(\begin{array}{l}
n \\
2
\end{array}\right)+o\left(n^{2}\right) .
$$

This means that $\operatorname{ex}(n, \mathcal{L})$ depends only very

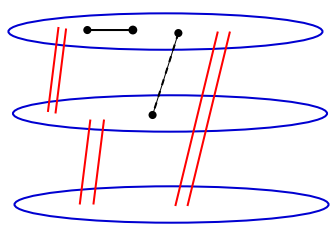
loosely on $\mathcal{L}$; up to an error term of order $o\left(n^{2}\right)$; it is already determined by $p(\mathcal{L})$. 11 The question is whether the structure of the extremal graphs is also almost determined by $p(\mathcal{L})$, and (therefore) it must be very similar to that of $T_{n, p} 12$. The answer is YES. This is expressed by the following results of Erdös and Simonovits [66], 67], 218]:

Theorem 2.3 (The Asymptotic Structure Theorem). Let $\mathcal{L}$ be a family of forbidden graphs with subchromatic number $p$. If $S_{n} \in \mathbf{E X}(n, \mathcal{L})$, (i.e, $S_{n}$ is extremal for $\mathcal{L})$, then it can be obtained from $T_{n, p}$ by deleting and adding $o\left(n^{2}\right)$ edges. Furthermore, if $\mathcal{L}$ is finite, then the minimum degree

$$
d_{\min }\left(S_{n}\right)=\left(1-\frac{1}{p}\right) n+o(n) .
$$

Further, the almost-extremal graphs are similar to $T_{n, p}$.

Theorem 2.4 (The First Stability Theorem). Let $\mathcal{L}$ be a family of forbidden graphs with subchromatic number $p$. For every $\varepsilon>0$, there exist a $\delta>0$ and an $n_{\varepsilon}$ such that, if $G_{n}$ contains no $L \in \mathcal{L}$, and if, for $n>n_{\varepsilon}$,

$$
e\left(G_{n}\right)>\operatorname{ex}(n, \mathcal{L})-\delta n^{2},
$$

then $G_{n}$ can be obtained from $T_{n, p}$ by changing 13 at most $\varepsilon n^{2}$ edges.

Remark 2.5. For ordinary graphs $(r=2)$ we often call the degenerate extremal graph problems bipartite extremal problems. This is the case when $\mathcal{L}$ contains some bipartite graphs. There is a slight problem here: we shall also consider the case when not only some $L \in \mathcal{L}$ is bipartite but $\chi\left(G_{n}\right)=2$ is as well.

\footnotetext{
${ }^{11}$ Better error terms are proved e.g. in 218, however, this will not be discussed here.

${ }^{12}$ Actually, this was the original question; Theorem 2.2 was a partial answer to it.

${ }^{13}$ deleting and adding
} 


\subsection{The importance of the Degenerate Case}

There are several results showing that if we know sufficiently well the extremal graphs for the degenerate cases, then we can also reduce the nondegenerate cases to these problems.

\section{Exact Turán numbers, product conjecture}

We start with an illustration. Let $O_{6}=K(2,2,2)$ be the octahedron graph. Erdös and Simonovits proved that

Theorem 2.6 (Octahedron Theorem [91]). If $S_{n}$ is an extremal graph for the octahedron $O_{6}$ for $n$ sufficiently large, then there exist extremal graphs $G_{1}$ and $G_{2}$ for the circuit $C_{4}$ and the path $P_{3}$ such that $S_{n}=G_{1} \otimes G_{2}$ and $\left|V\left(G_{i}\right)\right|=\frac{1}{2} n+o(n), i=1,2$.

If $G_{1}$ does not contain $C_{4}$ and $G_{2}$ does not contain $P_{3}$, then $G_{1} \otimes G_{2}$ does not contain $O_{6}$. Thus, if we replace $G_{1}$ by any $H_{1}$ in $\mathbf{E X}\left(v\left(G_{1}\right), C_{4}\right)$ and $G_{2}$ by any $H_{2}$ in $\mathbf{E X}\left(v\left(G_{2}\right), P_{3}\right)$, then $H_{1} \otimes H_{2}$ is also extremal for $\mathrm{O}_{6}$.

More generally,

Theorem 2.7 (Erdős-Simonovits [91]). Let L be a complete $(p+1)$-partite graph, $L:=K\left(a, b, r_{3}, r_{4}, \ldots, r_{p+1}\right)$, where $r_{p+1} \geq r_{p} \geq \cdots \geq r_{3} \geq b \geq a$ and $a=2,3$. There exists an $n_{0}=n_{0}\left(a, b, \ldots, r_{p+1}\right)$ such that if $n>n_{0}$ and $S_{n} \in \mathbf{E X}(n, L)$, then $S_{n}=U_{1} \otimes U_{2} \otimes \ldots \otimes U_{p}$, where

1. $v\left(U_{i}\right)=n / p+o(n)$, for $i=1, \ldots, p$.

2. $U_{1}$ is extremal for $K_{a, b}$

3. $U_{2}, U_{3}, \ldots, U_{p} \in \mathbf{E X}\left(n, K\left(1, r_{3}\right)\right)$.

It follows that this theorem is indeed a reduction theorem.

Conjecture 2.8 (The Product Conjecture, Simonovits). Assume that $p(\mathcal{L})=$ $\min _{L \in \mathcal{L}} \chi(L)-1>1$. If for some constants $c>0$ and $\varepsilon \in(0,1)$

$$
\operatorname{ex}(n, \mathcal{L})>e\left(T_{n, p}\right)+c n^{1+\varepsilon},
$$

then there exist $p$ forbidden families $\mathcal{M}_{i}$, with

$$
p\left(\mathcal{M}_{i}\right)=1 \quad \text { and } \quad \max _{M \in \mathcal{M}_{i}} v(M) \leq \max _{L \in \mathcal{L}} v(L),
$$

such that for any $S_{n} \in \mathbf{E X}(n, \mathcal{L}), S_{n}=G_{1} \otimes \ldots \otimes G_{p}$, where $G_{i}$ are extremal for $\mathcal{M}_{i}$. 
This means that the extremal graphs $S_{n}$ are products of extremal graphs for some degenerate extremal problems (for $\mathcal{M}_{i}$ ), and therefore we may reduce the general case to degenerate extremal problems.

Remarks 2.9. (a) If we allow infinite families $\mathcal{L}$, then one can easily find counterexamples to this conjecture.

(b) If we allow linear error-terms, i.e. do not assume (2.4), then one can also find counterexamples, using a general theorem of Simonovits [221]; however, this is not trivial at all, see 223 .

(c) A weakening of the above conjecture would be the following: for arbitrary large $n$, in Conjecture 2.8 there are several extremal graphs, and for each $n>n_{\mathcal{L}}$, some of them are of product form, (but maybe not all of them) and the families $\mathcal{M}_{i}$ also may depend on $n$ a little.

Further sources to read: Griggs, Simonovits, and Thomas [127], Simonovits, 226].

\subsection{The asymmetric case of Excluded Bipartite graphs}

The degenerate extremal graph problems have three different forms:

Problem 2.10 (Three versions). (a) Ordinary extremal graph problems, where some bipartite or non-bipartite sample graphs are excluded, and we try to maximize $e\left(G_{n}\right)$ under this conditions.

(b) The bipartite case, where the host graph is $K(m, n)$ and we maximize $e\left(G_{n+m}\right)$ under the conditions that $G_{n+m} \subseteq K(m, n)$ and $G_{n+m}$ contains no $L \in \mathcal{L}$. (Here we may assume that all $L \in \mathcal{L}$ are bipartite.) In this case we often use the notation $\operatorname{ex}(m, n, \mathcal{L})$. If $m \leq n$ but $m>c n$ for some constant $c>0$, then the answer to this problems and to the problem of $\mathbf{e x}(n, \mathcal{L})$ are the same, up to a constant. If, however, we assume that $n$ is much larger than $m$, then some surprising new phenomena occur, see Section 14.2 .

(c) The asymmetric case. Color the vertices of the sample graphs $L$ in RED-BLUE and exclude only those $G_{n} \subseteq K(m, n)$ where the RED vertices of some $L \in \mathcal{L}$ are in the FIRST class of $K(m, n)$ : maximize $e\left(G_{n+m}\right)$ over the remaining graphs $G_{n+m} \subseteq K(m, n)$.

Denote the maximum number of edges in this third case by $\mathbf{e x}^{*}(m, n, \mathcal{L})$.

Remark 2.11. We have seen Zarankiewicz' problem (i.e. Problem 1.16). That corresponds to an asymmetric graph problem, (c). If we exclude in an $m \times n$ matrix both a $a \times b$ and an $b \times a$ submatrices of 1 's, that will correspond to a bipartite graph problem, (b). 
Conjecture 2.12 (Erdős, Simonovits [225]).

$$
\operatorname{ex}^{*}(n, n, \mathcal{L})=O(\operatorname{ex}(n, \mathcal{L}))
$$

The simplest case when we cannot prove this is $L=K(4,5)$.

Remark 2.13 (Matrix problems). Case (c) has also a popular matrix form where we consider $0-1$ matrices and consider an $m \times n$ matrix not containing a submatrix $A$. The question is: how many 1 -s can be in such a matrix. This problem has (at least) two forms: the unordered and ordered one. We return to the Ordered Case in Subsection 12.4.

\subsection{Reductions: Host graphs}

The following simple but important observation shows that there is not much difference between considering any graph as a "host" graph or only bipartite graphs.

Lemma 2.14 (Erdős' bipartite subgraph lemma). Every graph $G_{n}$ contains a bipartite subgraph $H_{n}$ with $e\left(H_{n}\right) \geq \frac{1}{2} e\left(G_{n}\right)$.

This lemma shows that there is not much difference between considering $K_{2 n}$ or $K_{n, n}$ as a host graph.

Corollary 2.15. If $\operatorname{ex}_{B}(n, \mathcal{L})$ denotes the maximum number of edges in an $\mathcal{L}$-free bipartite graph, then

$$
\operatorname{ex}_{B}(n, \mathcal{L}) \leq \mathbf{e x}(n, \mathcal{L}) \leq 2 \mathbf{e x}_{B}(n, \mathcal{L})
$$

Assume now that we wish to have an upper bound on $\operatorname{ex}(m, n, \mathcal{L})$, where $n \gg m$. One way to get such an upper bound is to partition the $n$ vertices into subsets of size $\approx m$. If, e.g, we know that $\operatorname{ex}(m, m, \mathcal{L}) \leq c m^{1+\gamma}$, then we obtain that

$$
\mathbf{e x}(m, n, \mathcal{L}) \leq \frac{n}{m} \cdot \mathbf{e x}(m, m, \mathcal{L}) \leq c n m^{\gamma} .
$$

This often helps, however, occasionally it is too weak. Erdős formulated

Conjecture 2.16. If $n>m^{2}$ then $\operatorname{ex}\left(m, n, C_{6}\right)=O(n)$.

Later this conjecture was made more precise, by Erdős, A. Sárközy and T. Sós, and proved by Györi, see Section 14.2 and [134].

We start with a trivial lemma. 
Lemma 2.17. Let $d$ be the average degree in $G_{n}$, i.e. $d:=2 e\left(G_{n}\right) / n$. Then $G_{n}$ contains a $G_{m}$ with $d_{\min }\left(G_{m}\right) \geq d / 2$.

To solve the cube-problem, Erdős and Simonovits used two reductions. The first one was a reduction to bipartite graphs, see Section 2.14. The other one eliminates the degrees are much higher than the average.

Definition 2.18 ( $\Delta$-almost-regularity). $G$ is $\Delta$-almost-regular if $d_{\max }(G)<$ $\Delta \cdot d_{\min }(G)$.

Theorem 2.19 ( $\Delta$-almost-regularization [90]). Let $e\left(G_{n}\right)>n^{1+\alpha}$, and $\Delta=$ $20 \cdot 2^{(1 / \alpha)^{2}}$. Then there is a $\Delta$-almost-regular $G_{m} \subset G_{n}$ for which

$$
e\left(G_{m}\right)>\frac{2}{5} m^{1+\alpha}, \quad \text { where } \quad m>n^{\alpha \frac{1-\alpha}{1+\alpha}},
$$

unless $n$ is too small.

This means that whenever we wish to prove that $\operatorname{ex}(n, \mathcal{L})=O\left(n^{1+\alpha}\right)$, we may restrict ourselves to bipartite $\Delta$-almost-regular graphs.

It would be interesting to understand the limitations of this lemma better. The next remark and problem are in this direction.

Remark 2.20. By the method of random graphs one can show 90 that for every $\Delta$ and $n$ and $\varepsilon>0$, there is $G_{n}$ with $e\left(G_{n}\right)=\left\lfloor n^{3 / 2}\right\rfloor$ which does not have a $\Delta$-almost-regular subgraph $G_{m}$ with $e\left(G_{m}\right)>\varepsilon \sqrt{n} m$.

Problem 2.21 (Erdős-Simnovits [90]). Is it true that for every $\Delta$ there exists an $\varepsilon>0$ such that every $G_{n}$, with $e\left(G_{n}\right)=\lfloor n \log n\rfloor$, contains a $\Delta$ almost-regular subgraph $G_{m}$, with $e\left(G_{m}\right)>\varepsilon m \log m$ where $m \rightarrow \infty$ when $n \rightarrow \infty$ ?

\subsection{Excluding complete bipartite graphs}

Certain questions from topology (actually, Kuratowski theorem on planar graphs) led to Zarankiewicz problem [254]. After some weaker results Kövári, T. Sós and Turán proved the following theorem, already mentioned in Section 1.1.

Theorem 2.22 (Kövári-T. Sós-Turán, [164]). Let $K_{a, b}$ denote the complete bipartite graph with $a$ and $b$ vertices in its color-classes. Then

$$
\mathbf{e x}\left(n, K_{a, b}\right) \leq \frac{1}{2} \sqrt[a]{b-1} \cdot n^{2-(1 / a)}+\frac{a-1}{2} n .
$$


Remarks 2.23. (a) If $a \neq b$ then (2.6) is better if we apply it with $a<b$.

(b) We know from Theorem 2.2 that $\operatorname{ex}(n, \mathcal{L})=o\left(n^{2}\right)$ if and only if $\mathcal{L}$ contains a bipartite $L$. Actually Claim 2.1 and Theorem 2.22 show that if $\operatorname{ex}(n, \mathcal{L})=o\left(n^{2}\right)$ then $\operatorname{ex}(n, \mathcal{L})=O\left(n^{2-c}\right)$, for some constant $c=c_{\mathcal{L}}>0$.

Conjecture 2.24 ([164], see also e.g. [70]). The upper bound in Theorem 1.2 is sharp:

$$
\operatorname{ex}\left(n, K_{a, b}\right)>c_{a, b} n^{2-(1 / a)} .
$$

Sketch of proof of Theorem 2.22. The number of $a$-stars $K_{a, 1}$ in a graph $G_{n}$ is $\sum\left(\begin{array}{c}d_{i} \\ a\end{array}\right)$ where $d_{1}, \ldots, d_{n}$ are the degrees in $G_{n}$. If $G_{n}$ contains no $K_{a, b}$ then at most $b-1$ of these $a$-stars can share the same set of endpoints. We obtain

$$
\sum\left(\begin{array}{l}
d_{i} \\
a
\end{array}\right)=\text { the number of } a-\operatorname{stars} \leq(b-1)\left(\begin{array}{l}
n \\
a
\end{array}\right) .
$$

Extending $\left(\begin{array}{l}n \\ a\end{array}\right)$ to all $x>0$ by

$$
\left(\begin{array}{l}
x \\
a
\end{array}\right):= \begin{cases}\frac{x(x-1) \ldots(x-a+1)}{a !} & \text { for } x \geq a-1, \\
0 & \text { otherwise }\end{cases}
$$

we have a convex function. Then Jensen's Inequality implies that, the left hand side in (2.7) is at least $n\left(\begin{array}{c}2 e(G) / n \\ a\end{array}\right)$, and the result follows by an easy calculation.

Remark 2.25. Slightly changing the above proof we get analogous upper bounds on $e\left(G_{n}\right)$ in all three cases of Problem 2.10.

We shall return to these questions in Sections 3.1, 3.4 where we shall discuss some improvements of the upper bound and also some lower bounds. Further sources to read: Guy [128], Znám: 257], 256], Guy-Znám [129].

\subsection{Probabilistic lower bound}

The theory of random graphs is an interesting, important, and rapidly developing subject. The reader wishing to learn more about it should either read the original papers of Erdős, e.g., 61, 62, Erdős and Rényi, e.g., 84, or some books, e.g., Bollobás, [27], Janson, Łuczak and Ruciński, [149], Molloy and Reed [192].

Theorem 2.26 (Erdös-Rényi First Moment method). Let $\mathcal{L}=\left\{L_{1}, \ldots, L_{t}\right\}$ be a family of graphs, and let

$$
c=\max _{j} \min _{H \subseteq L_{j}} \frac{v(H)}{e(H)}, \quad \gamma=\max _{j} \min _{H \subseteq L_{j}} \frac{v(H)-2}{e(H)-1},
$$


where the minimum is taken only for subgraphs $H$ where the denominator is positive.

(a) Let $G_{n}$ be a graph of order $n$ chosen uniformly, at random, from graphs with $E_{n}$ edges. For every $\varepsilon>0$ there exists $a \delta>0$ such that if $E_{n}<\delta n^{2-c}$, then the probability that $G_{n}$ contains at least one $L \in \mathcal{L}$ is at most $\varepsilon$.

(b) If we know only $E_{n}<\varepsilon n^{2-\gamma}$, then the probability that $G_{n}$ contains at least $\frac{1}{2} E_{n}$ copies of $L \in \mathcal{L}$ is at most $\varepsilon$.

This implies that

$$
\operatorname{ex}(n, \mathcal{L})>c_{\mathcal{L}} n^{2-\gamma} \geq c_{\mathcal{L}} n^{2-c}
$$

with $c \geq \gamma>0$ defined above.

Remarks 2.27. (a) A graph $L$ is called balanced if the minimum in (2.8), for $c$, is achieved for $H=L$. Erdős and Rényi formulated their result containing Theorem 2.26(a) only for balanced graphs $L$. The part we use is trivial from their proof.

(b) Later Bollobás extended the Erdős-Rényi theorem to arbitrary $\mathcal{L}$.

(c) Győri, Rothschild and Ruciński achieved the generality by embedding any graph into a balanced graph [136].

Corollary 2.28. If a finite $\mathcal{L}$ contains no trees 14 then for some $c_{\mathcal{L}}>0$, $\operatorname{ex}(n, \mathcal{L}) \geq c_{\mathcal{L}} n^{1+c}$.

Mostly the weaker Theorem 2.26(a) implies Corollary 2.28, it does, whenever $\mathcal{L}$ is finite and each $L \in \mathcal{L}$ contains at least two cycles in the same component. However, for cycles we need the stronger Theorem 2.26(b).

For example, for $L=K_{a, b}$ we have $c=a^{-1}+b^{-1}$. Then, for $c_{0}$ sufficiently small, the probability that a graph $G_{n}$ with $c_{0} n^{2-c}$ edges does not contain $K_{a, b}$ is positive. Hence

$$
\operatorname{ex}\left(n, K_{a, b}\right) \geq c_{0} n^{2-(1 / a)-(1 / b)} .
$$

Comparing this with the Kövári-T. Sós-Turán theorem (Theorem 2.22), we see that the exponent is sharp there, in some sense, if $a$ is fixed while $b \rightarrow \infty$.

\footnotetext{
${ }^{14}$ neither forests
} 
Proof of Theorem 2.26. Consider the random graph $G_{n}$ with $n$ labeled vertices, in which each edge occurs independently, with the same probability $p$. For each $L_{j}$, choose a subgraph $H_{j}$ which attains the inner minimum for $\gamma$, in (2.8). Let $h_{j}:=v\left(H_{j}\right), e_{j}:=e\left(H_{j}\right)$, and let $\alpha_{j}$ denote the number of copies of $H_{j}$ in $K_{h_{j}}$, and $\beta_{j}$ denote the expected number of copies of $H_{j}$ in $G_{n}$.

Clearly, $K_{n}$ contains $\alpha_{j}\left(\begin{array}{c}n \\ h_{j}\end{array}\right)$ copies of $H_{j}$. For each copy $H$ of $H_{j}$, define a random "indicator" variable $k_{H}=k_{H}\left(G_{n}\right)=1$ if $H \subseteq G_{n}$, and 0 otherwise. Since the number of copies of $H_{j}$ in $G_{n}$ is just $\sum_{H \subseteq K_{n}} k_{H}$, therefore, if $\mathbb{E}$ denote the expected value, then

$$
\beta_{j}=\sum_{H \subseteq K_{n}} \mathbb{E}\left(k_{H}\right)=\alpha_{j}\left(\begin{array}{c}
n \\
h_{j}
\end{array}\right) p^{e_{j}} .
$$

Summing over $j$ and taking $p=c_{1} n^{-c}$, (for some $c_{1} \in(0,1)$ ) we get

$$
\sum_{j} \beta_{j} \leq t \max \alpha_{j}\left(\begin{array}{c}
n \\
h_{j}
\end{array}\right) p^{e_{j}} \leq t \max c_{1} n^{h_{j}-c e_{j}}=t c_{1} n^{2-c} .
$$

Now let $\eta\left(G_{n}\right)=e\left(G_{n}\right)-\sum_{j} \beta_{j}$. Then, for $c_{1}$ sufficiently small, the expected value is

$$
\mathbb{E}\left(\eta\left(G_{n}\right)\right)>\frac{1}{2}\left(\begin{array}{l}
n \\
2
\end{array}\right) p>\frac{1}{5} c_{1} n^{2-c} .
$$

Hence there exists a $G_{n}$ with $\eta\left(G_{n}\right)>\frac{1}{5} c_{1} n^{2-c}$. Delete an edge from each $H_{j}$ in this $G_{n}$. The resulting graph contains no $L_{j}$, and has at least $\frac{1}{5}\left(\begin{array}{l}n \\ 2\end{array}\right) p \geq$ $\frac{1}{11} c_{1} n^{2-c}$ edges, completing the proof.

Remarks 2.29 (How did the probabilistic methods start?). Mostly we write that applications of the Random Graphs (probabilistic method) started when Erdös (disproving a conjecture of Turán on the Ramsey Numbers) proved the existence of graphs $G_{n}$ without complete subgraphs of order $2 \log n$ and without independent sets of size $2 \log n$.

1. Erdös himself remarks (e.g., in [64]) that perhaps Szele was the first who applied this method in Graph Theory. (Erdős - in his birthday volume [76] - also mentions an even earlier application of J. Eröd but we did not succeed in locating that source.)

2. Perhaps the earliest case of applying probabilistic methods was that of Paul Turán's proof of the Hardy-Ramanujan Theorem [241, where reading the paper - it is obvious that Turán gave a probabilistic proof of a beautiful and important theorem, using the Chebishev inequality. 
However, either Turán did not realize that this is an application of the probabilistic method or he did not wish to burden the reader with that.

3. An important application of the probabilistic methods was that of Claude Shannon, when he constructed random codes.

Applying Theorem 2.26 to some families of cycles we obtain

Corollary 2.30. For some constant $c_{m}>0$,

$$
\operatorname{ex}\left(n,\left\{C_{3}, \ldots, C_{m}\right\}\right) \geq c_{m} n^{1+\frac{1}{m-1}} .
$$

Erdös' even cycles theorem asserts that $\mathbf{e x}\left(n, C_{2 t}\right)=O\left(n^{1+(1 / t)}\right)$, and this upper bound is probably sharp. 15 The random method (that is, Theorem [2.26) yields a lower bound of $c n^{1+\frac{1}{2 t-1}}$, a weaker result. Simonovits thinks that it is unlikely that Theorem 2.26 ever yields a sharp bound for a finite family. 16

Corollary 2.30 is used in the next section to prove that $\operatorname{ex}(n, \mathcal{L})=O(n)$ if and only if contains a tree or forest.

\subsection{Classification of extremal problems}

The extremal graph problems can be classified in several ways. Here we shall speak of (a) non-degenerate, (b) degenerate and (c) linear extremal problems.

For Case (a) Theorem 2.3 provides an appropriately good description of the situation. In Case (b) $p(\mathcal{L})=1$. Here the "main term" disappears, $\left(1-\frac{1}{p}\right)=0$; therefore "the error terms dominate". Case (c) will be discussed here shortly and in Sections 6 and 9 in more details.

The classification immediately follows from the following theorems:

Theorem 2.31. $\operatorname{ex}(n, \mathcal{L})=o\left(n^{2}\right)$ if and only if $\mathcal{L}$ contains a bipartite graph. Actually, if $\mathcal{L}$ contains a bipartite graph then $\operatorname{ex}(n, \mathcal{L})=O\left(n^{2-c}\right)$ for, e.g., $c=2 / v(L)$ for any bipartite $L \in \mathcal{L}$. If $\mathcal{L}$ does not contain bipartite graphs, then $\operatorname{ex}(n, \mathcal{L}) \geq\left[\frac{n^{2}}{4}\right]$.

\footnotetext{
${ }^{15}$ The reference is missing here, since Erdős did formulate this theorem but never have published a proof of it, as far as we know.

${ }^{16}$ Some related results of G. Margulis, and A. Lubotzky, R. Phillips and P. Sarnak will be discussed in Section 4.9
} 
Theorem 2.32. For finite $\mathcal{L}, \operatorname{ex}(n, \mathcal{L})=O(n)$ if and only if $\mathcal{L}$ contains a tree, or a forest. If $L \in \mathcal{L}$ is a tree or a forest, then, for $v(L) \geq 3$,

$$
\operatorname{ex}(n, \mathcal{L})<(v(L)-2) n .
$$

Theorem 2.33 (Erdős [61, 62]). If $\mathcal{L}$ is finite and no $L \in \mathcal{L}$ is a tree, then $\operatorname{ex}(n, \mathcal{L})>n^{1+c_{\mathcal{L}}}$ for some $c_{\mathcal{L}}>0$.

Theorem 2.34 (Erdős [62], Bondy and Simonovits [32]). Given an integer $k$, for some constants $c_{k}, \tilde{c}_{k}>0$,

$$
c_{k} n^{1+\frac{1}{2 k-1}}<\operatorname{ex}\left(n,\left\{C_{3}, \ldots, C_{2 k}\right\}\right) \leq \mathbf{e x}\left(n, C_{2 k}\right) \leq \tilde{c}_{k} n^{1+\frac{1}{k}}
$$

Proof of Theorems 2.31, 2.32, and 2.33, If there is a bipartite $L \in$ $\mathcal{L}$, then Theorem 2.22 implies the sharper upper bound of Theorem 2.31. Indeed, for $v=v(L)$, by $L \subseteq K([v / 2], v)$, we have,

$$
\operatorname{ex}(n, \mathcal{L}) \leq \mathbf{e x}(n, L) \leq \mathbf{e x}(n, K([v / 2], v))<\frac{1}{2} \sqrt[v]{2 v} \cdot n^{2-(2 / v(L))}=O\left(n^{2-c}\right) .
$$

If the minimum chromatic number $p=p(\mathcal{L}) \geq 3$, then $T_{n, p}$ contains no forbidden $L \in \mathcal{L}$. Therefore

$$
\operatorname{ex}(n, \mathcal{L})>e\left(T_{n, 2}\right) \geq e\left(T_{n, p}\right)=\left(1-\frac{1}{p}\right)\left(\begin{array}{l}
n \\
2
\end{array}\right)+O(n) .
$$

Actually, $e\left(T_{n, 2}\right)=\left[\frac{n^{2}}{4}\right]$. This completes the proof of Theorem 2.31

It is easy to show that if $G_{n}$ has minimum degree at least $r-1$, then it contains every tree $T_{r}$ (by induction on $r$ ). An induction on $n$ yields (2.10), implying half of Theorem 2.32, when $\mathcal{L}$ contains a tree (or a forest). If $\mathcal{L}$ is finite and contains no trees, i.e., all the forbidden graphs contain some cycles, then we use Theorem 2.34, or simply Corollary 2.28, proved by probabilistic methods 17

Remark 2.35 (Infinite families). For infinite families the situation is different: if e.g. $\mathcal{C}$ is the family of all cycles, then $\operatorname{ex}(n, \mathcal{C})=n-1$ : all graphs but the forests are excluded. There are many further families without trees where the extremal number is linear, see Section 9 .

\footnotetext{
${ }^{17}$ There are also deterministic proofs of Corollary 2.28, e.g., via the Margulis-LubotzkyPhillips-Sarnak construction of Ramanujan graphs, see Construction 4.43
} 
Proof of Theorem 2.34, The lower bound comes from a random graph argument of Erdős. Concentrate on the upper bound. If we are not interested in the value of the constant, then we can basically use the following argument: Take a graph $G_{n}$ with $c n^{1+\alpha}$ edges. Delete its minimum degree vertex, then the minimum degree vertex in the remaining graph, etc. At the end we get a $G_{m}$ with minimum degree at least $c_{1} m^{\alpha}$. In the obtained graph $G_{m}$ fix a vertex $x$ and denote by $S_{j}$ the set of vertices at distance $j$ from $x$. If $\operatorname{girth}\left(G_{n}\right)>2 k,-$ as we assumed - then basically $\left|S_{j}\right|>d_{\min }\left(G_{m}\right) \cdot\left|S_{j-1}\right|$. Hence $m>\left|S_{k}\right|>c_{1}^{k} m^{\alpha k}$. So $\alpha \leq 1 / k$.

Assume for a second that $G_{n}$ itself is asymptotically regular:

$$
\frac{d_{\min }\left(G_{n}\right)}{d_{\max }\left(G_{n}\right)} \rightarrow 1
$$

Then the previous argument asserts that $d:=d_{\min }\left(G_{n}\right)<n^{1 / k}$. Therefore

$$
e\left(G_{n}\right) \leq\left(\frac{1}{2}+o(1)\right) n d \approx \frac{1}{2} n^{1+\frac{1}{k}}
$$

We shall return to the case of excluded trees, namely, to the Erdős-Sós conjecture on the extremal number of trees, and to the related KomlósSós conjecture in Section 6. One final question could be if $\operatorname{ex}(n, \mathcal{L})$ can be sublinear. This is answered by the following trivial result.

Theorem 2.36. If $\mathcal{L}$ is finite and $\operatorname{ex}(n, \mathcal{L})<[n / 2]$, then $\operatorname{ex}(n, \mathcal{L})=O(1)$.

Proof. Consider $n / 2$ independent edges: this must contain an $L_{1} \in \mathcal{L}$. Hence, there is an $L_{1} \in \mathcal{L}$ contained in the union of $t$ independent edges, for some $t$. Also, there exists an $L_{2} \subseteq K(1, n-1)$. Hence an extremal graph $S_{n}$ has bounded degrees and bounded number of independent edges. This proves 2.36 .

Theorem 2.36 easily extends to hypergraphs.

\subsection{General conjectures on bipartite graphs}

We have already formulated Conjecture 1.6 on the rational exponents. We have to remark that for hypergraphs this does not hold: the Behrend construction [21] is used to get lower bounds in the Ruzsa-Szemerédi Theorem, (Thm 1.9), showing that there is no rational exponent in that case. Yet,

Erdös and Simonovits conjectured that for ordinary graphs there is. One could also conjecture the inverse extremal problem: 
Conjecture 2.37. For every rational $\alpha \in(0,1)$ there is a finite $\mathcal{L}$ for which $c_{1} n^{1+\alpha}<\operatorname{ex}(n, \mathcal{L})<c_{2} n^{1+\alpha}$, for some constants $c_{1}, c_{2}>0$.

The third conjecture to be mentioned here is on "compactness" 93]:

Conjecture 2.38. For every finite $\mathcal{L}$ there is an $L \in \mathcal{L}$ for which $\operatorname{ex}(n, \mathcal{L})>$ $c \cdot \operatorname{ex}(n, L)$, for some constants $c=c_{\mathcal{L}}>0$.

\section{Excluding complete bipartite graphs}

\subsection{Bipartite $C_{4}$-free graphs and the Zarankiewicz problem}

Turán type extremal results (and Ramsey results as well) can often be applied in Mathematics, even outside of Combinatorics. Turán himself explained this applicability by the fact that - in his opinion - the extremal graph results were generalizations of the Pigeon Hole Principle.

Recall that $Z(m, n, a, b)$ denotes the maximum number of 1 's in an $m \times n$ matrix not containing an $a \times b$ minor consisting exclusively of 1 's. In 1951 Zarankiewicz 254] posed the problem of determining $Z(n, n, 3,3)$ for $n \leq 6$, and the general problem has also become known as the problem of Zarankiewicz 18 Obviously, $Z(m, n, 1, b)=m(b-1)$ (for $n \geq b-1)$. Observe that $Z(m, n, a, b)=\mathbf{e x}^{*}\left(m, n, K_{a, b}\right)$ (where $\mathbf{e x}^{*}(m, n, \mathcal{L})$ was defined following Remark 2.10.) Considering the adjacency matrix of a $K_{a, b}$-free graph on $n$ vertices we get $2 \operatorname{ex}\left(n, K_{a, b}\right) \leq Z(n, n, a, b)$. We will use this upper bound many times.

We will see that the easy upper bound in Theorem 2.22 is pretty close to the truth for $a \leq 2$. Actually, Kövári, T. Sós and Turán [164 proved an upper bound for the Zarankiewicz function

$$
Z(m, n, a, b) \leq \sqrt[a]{b-1} \cdot m n^{1-(1 / a)}+(a-1) n
$$

which was slightly improved by Znám [257], 256], (he halved the last term to $(a-1) n / 2$ in the case of $m=n)$ and Guy 128 .

A bipartite graph $G[M, N]$ where $|M|=m,|N|=n$ is $C_{4}$-free if its "bipartite" $m \times n$ adjacency matrix contains no $2 \times 2$ full 1 submatrix 19 In other terminology, the hypergraph defined by the rows of this matrix is

\footnotetext{
${ }^{18}$ In Graph Theory two problems are connected to Zarankiewicz' name: the extremal problem for matrices that we shall discuss here and the Crossing Number conjecture which is not our topic. Actually, the crossing number problem comes from Paul Turán, see [244].

${ }^{19}$ Here the "bipartite adjacency matrix" $A=\left(a_{i j}\right)_{m \times n}$ is defined for a bipartite graph $G[U, V]$ and $a_{i j}=1$ if $u_{j} \in U$ is joined to $v_{j} \in V$, otherwise $a_{i j}=0$.
} 
linear, and their hyperedges pairwise meet in at most one element. There is an important class of such hypergraphs, the Steiner $k$-systems $S(n, k, 2)$. A family $\mathcal{S}$ of $k$-subsets of an $n$-set $N$ is a Steiner $k$-system if every pair of elements is covered exactly once. For such an $\mathcal{S}$, clearly, $|\mathcal{S}|=m=\left(\begin{array}{l}n \\ 2\end{array}\right) /\left(\begin{array}{l}k \\ 2\end{array}\right)$. Such families are known to exists for $(m, n, k)=\left(q^{2}+q+1, q^{2}+q+1, q+1\right)$ (called finite projective planes of order $q)$, and $(m, n, k)=\left(q^{2}+q, q^{2}, q\right)$ (affine planes) whenever $q$ is a power of a prime. Also for any given $k$ there exists an $n_{0}(k)$ such that $S(n, k, 2)$ exists for all admissible $n>n_{0}(k)$, i.e., when $(n-1) /(k-1)$ and $n(n-1) / k(k-1)$ are integers (Wilson's existence theorem [248]).

Kövári, T. Sós and Turán [164] proved that

Theorem 3.1. $Z(n, n, 2,2)=(1+o(1)) n^{3 / 2}$, and

$$
Z(n, n, 2,2)<\left[n^{3 / 2}\right]+2 n .
$$

Further, if $p$ is a prime, then

$$
Z\left(p^{2}+p, p^{2}, 2,2\right)=p^{3}+p^{2} .
$$

Reiman [206] returned to this topic, (see also [207]), slightly improving (3.2)

Theorem 3.2 (Reiman [206]).

$$
Z(m, n, 2,2) \leq \frac{1}{2}\left(m+\sqrt{m^{2}+4 m n(n-1)}\right) .
$$

For large $m, n \rightarrow \infty$, and $m=o\left(n^{2}\right)$, this yields

$$
Z(m, n, 2,2) \leq\left(\frac{1}{2}+o(1)\right) n \sqrt{m} .
$$

Further, for $m=n$, we get

$$
Z(n, n, 2,2) \leq \frac{1}{2} n(1+\sqrt{4 n-3}) \approx n \sqrt{n} .
$$

Reiman also provides infinitely many graphs, using Finite Geometries, showing the sharpness of (3.3) and (3.4). We have equality when $m=n(n-$ $1) / k(k-1)$ and a Steiner system $S(n, k, 2)$ exists. Thus he determined the case

$$
Z(n, n, 2,2)=\frac{1}{2} n(1+\sqrt{4 n-3})=\left(q^{2}+q+1\right)(q+1)
$$


for $m=n=q^{2}+q+1$ when a projective plane of order $q$ exists. Actually, in [207, Reiman also speaks about Zarankiewicz-extremal graphs connected to incidence-graphs of higher dimensional finite geometries.

Since Reiman's theorem the theory of finite geometries developed tremendously. We cite here a recent result whose proof used the most modern tools and stability results.

Theorem 1 (Damásdi, Héger, and Szönyi [57]). Let $q \geq 15$, and $c \leq q / 2$. Then

$$
Z\left(q^{2}+q+1-c, q^{2}+q+1,2,2\right) \leq\left(q^{2}+q+1-c\right)(q+1) .
$$

Equality holds if and only if a projective plane of order $q$ exists. Moreover, graphs giving equality are subgraphs of the bipartite incidence graph of a projective plane of order $q$ obtained by omitting $c$ rows of its incidence matrix.

They proved many more exact results when a projective plane of order $q$ exists. The extremal configurations are submatrices of the incidence matrix of a projective plane.

$$
\begin{aligned}
Z\left(q^{2}+c, q^{2}+q, 2,2\right) & =q^{2}(q+1)+c q \quad(0 \leq c \leq q+1), \\
Z\left(q^{2}-q+c, q^{2}+q-1,2,2\right) & =\left(q^{2}-q\right)(q+1)+c q \quad(0 \leq c \leq 2 q), \\
Z\left(q^{2}-2 q+1+c, q^{2}+q-2,2,2\right) & =\left(q^{2}-2 q+1\right)(q+1)+c q \quad(0 \leq c \leq 3(q-1)) .
\end{aligned}
$$

These refer to bipartite host graphs. As we will see later, such exact results are rare for the general (non-bipartite) case. To estimate $\mathbf{e x}\left(n, C_{4}\right)$ seems to be harder, because the corresponding 0-1 matrices, the incidence matrix of a graph, should be symmetric.

\subsection{Finite Geometries and the $C_{4}$-free graphs}

The method of finite geometric constructions is very important and powerful in combinatorics. In particular, it is often the best way to obtain lower bounds. It is for this reason that we include this section.

We give several constructions: the first two show that the Kövári-T. Sós-Turán theorem (Theorem 2.22) is sharp for both $K_{2,2}$ and $K_{3,3}$.

Remark 3.3. When we write that an upper bound is sharp, mostly we mean that it is sharp up to a multiplicative constant: it yields the correct exponent. There are a few exceptions, where sharpness means that the ratio of the upper and lower bounds tends to 1 . This is the case for $C_{4}=K_{2,2}$ 
and we have this also for $K_{3,3}$. Here, however, the matching upper bound for Construction 3.20 below is given not by Theorem 2.22 but by the Füredi improvement [112.

Perhaps the application of finite geometries in Extremal Graph Theory started in the Erdös paper, with the construction of Eszter Klein [60], to prove the sharpness of Theorem 1.15. The expression "Finite Geometry" was not mentioned there. We skip the description of this whole story, since it was described in several places, e.g., [227, [228].

Much later, Erdős and Rényi [85] used finite geometry for a diameterextremal problem. This is a very large area, connected to our problems, yet we have to skip it. The interested reader is refered to [85], (translated into English in [208]).

Sharp extremal graph results were obtained by Reiman [206] and a Polarity Graph was used in [86] and 36] to give asymptotically sharp lower bound on $\operatorname{ex}\left(n, C_{4}\right)$. This lower bound can also be found in [85, implicitly: Erdős and Rényi considered the diameter-extremal problem, and do mention the properties of this graph.

The real breakthrough came by the Erdős-Rényi-T. Sós paper [86], (sharp lower bound for $C_{4}$ ) and by the Brown paper [36], providing asymptotically sharp lower bounds for $\operatorname{ex}\left(n, C_{4}\right)$ and for $\operatorname{ex}\left(n, K_{3,3}\right)$. (See Remark 3.3.)

We know from Theorem 2.22 that $\operatorname{ex}\left(n, C_{4}\right) \leq \frac{1}{2} n^{3 / 2}+o\left(n^{3 / 2}\right)$, but is this result sharp? In analyzing the proof, we realize that if it is sharp (that is, if there are infinitely many graphs $G_{n}$ not containing $C_{4}$ and having $\approx \frac{1}{2} n \sqrt{n}$ edges), then almost all degrees are $\approx \sqrt{n}$ and almost every pair of vertices must have a common neighbor (and no pair has two). This suggests that the neighborhoods $N(x)$ behave much like the lines in a projective plane, in that the following statement "almost" holds: any two vertices lie in a common set, and any two sets intersect in one vertex.

Theorem 3.4 (Erdős-Rényi-T. Sós [86], and Brown [36], see also [164]).

$$
\operatorname{ex}\left(n, C_{4}\right)=\frac{1}{2} n^{3 / 2}+O\left(n^{3 / 2-c}\right) .
$$

For the lower bound for $\mathbf{e x}\left(n, C_{4}\right)$ we use the following

Construction 3.5. Let $p$ be a prime, $n=p^{2}-1$. Construct a graph as follows: the vertices are the $p^{2}-1$ non-zero pairs $(x, y)$ of residues (modulo $p)$, and $(x, y)$ is joined to $(a, b)$ by an edge if $a x+b y=1$. (This graph may contain loops, but we simply delete them.) 
With $n=p^{2}-1$, the resulting graph $H_{n}$ has the necessary properties to show the sharpness of Theorem 2.22 for $C_{4}$ :

(a) for a given pair $(a, b)$, mostly there are $p$ solutions to $a x+b y=1$, so that, even after the loops are deleted, there are at least $\frac{1}{2}\left(p^{2}-1\right)(p-1)$ edges in $H_{n}$ and hence $e\left(H_{n}\right)>\frac{1}{2} n^{3 / 2}-n$;

(b) if $H_{n}$ had a 4 -cycle with vertices $(a, b),(u, v),\left(a^{\prime}, b^{\prime}\right)$ and $\left(u^{\prime}, v^{\prime}\right)$, then the two equations $a x+b y=1$ and $a^{\prime} x+b^{\prime} y=1$ would have two solutions, which is impossible. Since the primes are "dense" among the integers, this completes the proof of the the sharpness of Theorem 2.22 for $a=b=2$.

Remark 3.6. An alternative possibility is to use the much more symmetric polarity graph of the projective plane (we explain this in the next section): here we used the Affine Geometric Variant because here we did not wish to use anything from Projective Geometry.

\subsection{Excluding $C_{4}$ : Exact results}

The polarity graph 20, used in [85], was also used in [86] and [36] to prove that

$$
\operatorname{ex}\left(n, C_{4}\right) \geq \frac{1}{2} q(q+1)^{2}, \quad \text { for } \quad n=q^{2}+q+1 .
$$

if $q$ is a prime power.

Construction 3.7 (The Polarity Graph from the finite field). Assume that $q$ is a prime power. Consider the Finite Field $G F(q)$. The vertices of our graph are the equivalence classes of the non-zero triples $(a, b, c) \in$ $G F(q)^{3}$ where two of them, $(a, b, c)$ and $\left(a^{\prime}, b^{\prime}, c^{\prime}\right)$ are considered the same if $\left(a^{\prime}, b^{\prime}, c^{\prime}\right)=\lambda(a, b, c)$ for some $\lambda \neq 0$. There are $\left(q^{3}-1\right) /(q-1)=q^{2}+q+1$ such classes. Further, the equivalence class of $(a, b, c)$ is connected by an edge to the class of $(x, y, z)$ if $a x+b y+c z=0$. Finally, we delete the $q+1$ loops, i.e. those edges, where $a^{2}+b^{2}+c^{2}=0$. This graph is $C_{4}$-free and it has $\frac{1}{2}(n(q+1)-(q+1))$ edges.

In general, a polarity corresponds to a symmetric incidence matrix of a finite plane of size $\left(q^{2}+q+1\right) \times\left(q^{2}+q+1\right)$. According to a theorem of Baer [13] such a matrix has at least $q+1$ non-zero elements in its diagonal. Therefore using the polarity graph we cannot avoid losing on loops. This way Erdős,

${ }^{20}$ These $C_{4}$-free graphs were studied earlier in finite geometry. The bipartite pointline incidence graph appeared in Levi's book (1942) and polarity graphs (modulo loops) obtained from Levi graph had been described already by Artzy (1956). For more details and references see Bondy [30]. 
Rényi and Sós [86] showed that indeed $\mathbf{e x}\left(q^{2}+q+1, C_{4}\right)<\frac{1}{2} n(q+1)$. Yet one could hope to get a better construction. Erdős conjectured 66, [71 that there are no better constructions, that is, (3.6) is sharp if $n=q^{2}+q+1,(q$ is a prime power).

Füredi settled this conjecture in the following sense: First he proved 103 that if $q=2^{k}$, then Erdös' conjecture holds. Next he settled the case $q \geq q_{0}$. Later he found a much shorter proof of the weaker assertion that the Polarity graphs are extremal; however, this shorter version did not give the extremal structure. So Füredi published the shorter version, while the longer version can be found on his homepage.

Theorem 3.8 (Füredi [111, [104]). If $q \neq 1,7,9,11,13$ and $n=q^{2}+q+1$, then $\mathbf{e x}\left(n, C_{4}\right) \leq \frac{1}{2} q(q+1)^{2}$ and for $q>13$ the extremal graphs are obtained from a polarity of a finite projective plane. Hence if $q>13$ is a prime power, then $\mathbf{e x}\left(n, C_{4}\right)=\frac{1}{2} q(q+1)^{2}$.

The second part of this result probably holds for $q \in\{7,9,11,13\}$, too.

Recently a new sharp construction has been found for $n=q^{2}+q$.

Theorem 3.9 (Firke, Kosek, Nash and Williford [102]). Suppose that $q$ is even, $q>q_{0}$. Then

$$
\operatorname{ex}\left(q^{2}+q, C_{4}\right) \leq \frac{1}{2} q(q+1)^{2}-q .
$$

Consequently, if $q>q_{0}, q=2^{k}$ and $n=q^{2}+q$ then $\operatorname{ex}\left(n, C_{4}\right)=q(q+1)^{2}-q$.

They also announced that in a forthcoming paper they show that for all but finitely many even $q$, any $S_{n} \in \mathbf{E X}\left(q^{2}+q, C_{4}\right)$ is derived from an orthogonal polarity graph by removing a vertex of minimum degree (the 1-vertex-truncated Polarity graph, see Construction 3.7). This result shows a kind of stability of the Polarity graph. More generally, McCuaig (private communication, 1985) conjectured that each extremal graph is a subgraph of some polarity graph. So this is true for infinitely many cases, but one of the present authors strongly disagrees and he believes just the opposite that for e.g., $n=q^{2}+q+2$ maybe the extremal graphs are obtained by adding an extra vertex and some edges to a polarity graph.

Remark 3.10. W. McCuaig calculated $\operatorname{ex}\left(n, C_{4}\right)$ for $n \leq 21$ (unpublished letter, 1985). Clapham, Flockart and Sheehan determined the corresponding extremal graphs [48, and Yuansheng and Rowlinson [252], - using computers, - extended these results to $n \leq 31$. (They also determined the graphs in $\mathbf{E X}\left(n, C_{6}\right)$ for $n \leq 26$, 253.) Garnick, Kwong, Lazebnik, and Nieuwejaar [122, [123] determined the values of $\operatorname{ex}\left(n,\left\{C_{3}, C_{4}\right\}\right)$ for all $n \leq 30$. 


\subsection{Excluding $K(2, t+1), t>1$}

A slightly sharper form of the upper bound (3.1) was presented by HylténCavallius [146]

$$
Z(m, n, 2, k) \leq \frac{1}{2} n+\left\{(k-1) n m(m-1)+\frac{1}{4} n^{2}\right\}^{1 / 2} .
$$

Obviously, for fixed $k$ and large values of $n, m$, if $n=o\left(m^{2}\right)$, then the right hand side of (3.7) is $\approx \sqrt{k-1} m \sqrt{n}$. Using again the observation $2 \operatorname{ex}\left(n, K_{2, t+1}\right) \leq Z(n, n, 2, t+1)$ one obtains the upper bound

$$
\operatorname{ex}\left(n, K_{2, t+1}\right) \leq \frac{1}{2} n \sqrt{t n-t+1 / 4}+(n / 4) .
$$

The following theorem shows that the above (easy) upper bound is the best possible asymptotically.

Theorem 3.11 (Füredi [113]). For any fixed $t \geq 1$

$$
\operatorname{ex}\left(n, K_{2, t+1}\right)=\frac{1}{2} \sqrt{t} n^{3 / 2}+O\left(n^{4 / 3}\right) .
$$

To prove this Theorem one needs an appropriate lower bound, a construction. Let $q$ be a prime power such that $(q-1) / t$ is an integer. We will construct a $K_{2, t+1}$-free graph $G$ on $n=\left(q^{2}-1\right) / t$ vertices such that every vertex has degree $q$ or $q-1$. We will explain this below (Construction 3.15). Then $G$ has more than $(1 / 2) \sqrt{t} n^{3 / 2}-(n / 2)$ edges. The gap between the lower and upper bounds is only $O(\sqrt{n})$ for $n=\left(q^{2}-1\right) / t$. The lower bound for the Turán number for all $n$ then follows from the fact that such prime powers form a dense subsequence among the integers. This means that for every sufficiently large $n$ there exists a prime $q$ satisfying $q \equiv 1(\bmod t)$ and $\sqrt{n t}-n^{1 / 3}<q<\sqrt{n t}$ (see [145]).

Construction 3.15 below is inspired by constructions of Hyltén-Cavallius and Mörs given for Zarankiewicz's problem $Z(n, n, 2, t+1)$.

Theorem 3.12 (Hyltén-Cavallius [146]). $Z(n, n, 2,3)=\sqrt{2} n^{3 / 2}+o\left(n^{3 / 2}\right)$. Also

$$
\sqrt{\lfloor k / 2\rfloor} \leq \liminf _{n \rightarrow \infty} \frac{Z(n, n, 2, k)}{n^{3 / 2}} .
$$

Theorem 3.13 (Mörs [194]). For all $t \geq 1$,

$$
\frac{Z(n, n, 2, t+1))}{n^{3 / 2}} \rightarrow \sqrt{t}, \quad \text { as } \quad n \rightarrow \infty
$$


The topic was so short of constructions that, as a first step, P. Erdős [66, 69] even proposed the problem whether $\lim _{t}\left(\liminf _{n} \mathbf{e x}\left(n, K_{2, t+1}\right) n^{-3 / 2}\right)$ goes to $\infty$ as $t \rightarrow \infty$.

Remark 3.14. Here we see three distinct quantities, exactly as it is described in Problem 2.10, $Z(m, n, 2, t+1)=\mathbf{e x}^{*}\left(m, n, K_{2, t+1}\right)$, estimated from below by Mörs, by a construction, and $\mathbf{e x}\left(m, n, K_{2, t+1}\right)$ estimated by Füredi by the same construction. Füredi showed that the matrix of Mörs contains neither a $(t+1) \times 2$ submatrix, nor a $2 \times(t+1)$ submatrix of 1 's; finally, Füredi, slightly changing the definitions in Mörs's construction extended this "asymmetric matrix" result to the symmetric case and provided a non-bipartite graph, proving (3.9).

Construction 3.15. Let $G F(q)$ be the q-element finite field, and let $h \in G F(q)$ be an element of order $t$. This means, that $h^{t}=1$ and the set $H=$ $\left\{1, h, h^{2}, \ldots, h^{t-1}\right\}$ form a t-element subgroup of $G F(q) \backslash\{0\}$. For $q \equiv 1$ $(\bmod t)$ such an element $h \in G F(q)$ always exists.

We say that $(a, b) \in G F(q) \times G F(q),(a, b) \neq(0,0)$ is equivalent to $\left(a^{\prime}, b^{\prime}\right)$, in notation $(a, b) \sim\left(a^{\prime}, b^{\prime}\right)$, if there exists some $h^{\alpha} \in H$ such that $a^{\prime}=h^{\alpha} a$ and $b^{\prime}=h^{\alpha} b$. The elements of the vertex set $V$ of $G$ are the $t$ element equivalence classes of $G F(q) \times G F(q) \backslash(0,0)$. The class represented by $(a, b)$ is denoted by $\langle a, b\rangle$. Two (distinct) classes $\langle a, b\rangle$ and $\langle x, y\rangle$ are joined by an edge in $G$ if $a x+b y \in H$. This relation is symmetric, and $a x+b y \in H,(a, b) \sim\left(a^{\prime}, b^{\prime}\right)$, and $(x, y) \sim\left(x^{\prime}, y^{\prime}\right)$ imply $a^{\prime} x^{\prime}+b^{\prime} y^{\prime} \in H$. So this definition is compatible with the equivalence classes.

For any given $(a, b) \in G F(q) \times G F(q) \backslash(0,0)$ (say, $b \neq 0)$ and for any given $x$ and $h^{\alpha}$, the equation $a x+b y=h^{\alpha}$ has a unique solution in $y$. This implies that there are exactly $t q$ solutions $(x, y)$ with $a x+b y \in H$. The solutions come in equivalence classes, so there are exactly $q$ classes $\langle x, y\rangle$. One of these classes might coincide with $\langle a, b\rangle$ so the degree of the vertex $\langle a, b\rangle$ in $G$ is either $q$ or $q-1$.

We claim that $G$ is $K_{2, t+1}$-free. First we show, that for $(a, b),\left(a^{\prime}, b^{\prime}\right) \in$ $G F(q) \times G F(q) \backslash(0,0),(a, b) \nsim\left(a^{\prime}, b^{\prime}\right)$ the equation system

$$
a x+b y=h^{\alpha} \quad \text { and } \quad a^{\prime} x+b^{\prime} y=h^{\beta}
$$

has at most one solution $(x, y) \in G F(q) \times G F(q) \backslash(0,0)$. Indeed, the solution is unique if the determinant $\operatorname{det}\left(\begin{array}{cc}a & b \\ a^{\prime} & b^{\prime}\end{array}\right)$ is not 0 . Otherwise, there exists a $c$ such that $a=a^{\prime} c$ and $b=b^{\prime} c$. If there exists a solution of (2) at all, then multiplying the second equation by $c$ and subtracting it from the first one 
we get on the right hand side $h^{\alpha}-c h^{\beta}=0$. Thus $c \in H$, contradicting the fact that $(a, b)$ and $\left(a^{\prime}, b^{\prime}\right)$ are not equivalent.

Finally, there are $t^{2}$ possibilities for $0 \leq \alpha, \beta<t$ in (3.10). The set of solutions again form $t$-element equivalence classes, so there are at most $t$ equivalence classes $\langle x, y\rangle$ joint simultaneously to $\langle a, b\rangle$ and $\left\langle a^{\prime}, b^{\prime}\right\rangle$.

Since then, there have been two additional almost optimal constructions, strongly related to the Construction 3.15 above.

Construction 3.16 (Lazebnik, Mubayi [167]). Let $G F(q)^{*}$ be the finite field of order $q$ without the zero element. Suppose $q \equiv 1(\bmod t)$ and let $H$ be the t-element multiplicative subgroup of $G F(q)^{*}$. Define the graph $G^{\times}$as follows. Let $V\left(G^{\times}\right)=\left(G F(q)^{*} / H\right) \times G F(q)$. For $\langle a\rangle,\langle b\rangle \in\left(G F(q)^{*} / H\right)$ and $x, y \in G F(q)$, make $(\langle a\rangle, x)$ adjacent to $(\langle b\rangle, y)$ if $x+y \in\langle a b\rangle$.

This graph (after deleting the eventual loops) is $K_{2, t+1}$-free and every vertex has degree $q-1$ or $q-2$. Actually, Construction 3.16 differs from Construction 3.15 only in that its vertex set is smaller and instead of using the rule that $\langle a, b\rangle$ is adjacent to $\langle x, y\rangle$ if $a x+b y \in H$ they use the rule $a y+b x \in H$. This change allows them to generalize it to multipartite hypergraphs.

The following example works only if $t$ is a power of a prime, and $t \mid q$.

Construction 3.17 (Lenz, Mubayi [173]). Suppose that $t$ divides $q$ and let $H$ be an additive subgroup of $G F(q)$ of order $t$. Define the graph $G^{+}$ as follows. Let $V\left(G^{+}\right)=(G F(q) / H) \times G F(q)^{*}$. We will write elements of $G F(q) / H$ as $\langle a\rangle$. It is the additive coset of $H$ generated by $a,\langle a\rangle=$ $\{h+a: h \in H\}$. For $\langle a\rangle,\langle b\rangle \in(G F(q) / H)$ and $x, y \in G F(q)^{*}$, make $(\langle a\rangle, x)$ adjacent to $(\langle b\rangle, y)$ if $x y \in\langle a+b\rangle$. (This, in fact, means that there exists an $h \in H$ such that $x y=a+b+h)$.

\subsection{Excluding $K(3,3)$, and improving the upper bound}

The main result of this section is the description of the asymptotically sharp value of $\mathbf{e x}\left(n, K_{3,3}\right)$.

Theorem 3.18 (Brown [36] and Füredi [112]).

$$
\operatorname{ex}\left(n, K_{3,3}\right)=\frac{1}{2} n^{5 / 3}+O\left(n^{(5 / 3)-c}\right) \quad \text { for some } \quad c>0 .
$$

The lower bound can be obtained from Brown's example (discussed below as Construction (3.20) who gave a $\left(p^{2}-p\right)$-regular $K_{3,3}$-free graph on $p^{3}$ vertices for each prime $p$ of the form $4 k-1$. 
Improving the upper bound in Theorem 2.22 Füredi showed that Brown's example is asymptotically optimal.

Theorem 3.19 (Füredi [112]). For all $m \geq a, n \geq b, b \geq a \geq 2$ we have

$$
Z(m, n, a, b) \leq(b-a+1)^{1 / a} m n^{1-(1 / a)}+(a-1) n^{2-(2 / a)}+(a-2) m .
$$

For fixed $a, b \geq 2$ and $n, m \rightarrow \infty$ the first term is the largest one for $n=O\left(m^{a /(a-1)}\right)$. This upper bound is asymptotically optimal for $a=2$ and for $a=b=3(m=n)$. We obtain

$$
\operatorname{ex}\left(n, K_{3,3}\right) \leq \frac{1}{2} Z(n, n, 3,3) \leq \frac{1}{2} n^{5 / 3}+n^{4 / 3}+\frac{1}{2} n .
$$

Alon, Rónyai and Szabó [11] gave an example (discussed as Construction 3.25) showing that

$$
\operatorname{ex}\left(n, K_{3,3}\right) \geq \frac{1}{2} n^{5 / 3}+\frac{1}{3} n^{4 / 3}-C .
$$

for some absolute constant $C>0$ for every $n$ of the form $n=p^{3}-p^{2}, p$ is a prime. Their example shows that the upper bound (3.12) (and (3.11)) is so tight that that we cannot leave out the second order term. It would be interesting to see whether (3.11) is tight for other values of $a$ and $b$, too.

The first step of the proof of Theorem 3.19 is that given a $K_{a, b}$-free graph $G$, we apply the original bound (3.1) to the bipartite subgraphs $G[N(x), V \backslash$ $N(x)$ ] generated by the neighborhood of a vertex $x$ and its complement.

When Brown gave his construction, the matching upper bound of Theorem 3.19 was not known yet. He wrote that even the existence of $\lim _{n \rightarrow \infty} \mathbf{e x}\left(n, K_{3,3}\right) / n^{5 / 3}$ was unknown.

Construction 3.20. Let $p$ be an odd prime $n=p^{3}$ and $d \in G F(p), d \neq 0 a$ quadratic residue if $p$ is of the form $4 k-1$ and $d$ be a non-residue otherwise. Construct a graph $B_{n}$ whose vertices are the triples $(x, y, z)$ of residue classes (modulo $p$ ) and whose edges join vertices $(x, y, z)$ and $\left(x^{\prime}, y^{\prime}, z^{\prime}\right)$ if

$$
\left(x-x^{\prime}\right)^{2}+\left(y-y^{\prime}\right)^{2}+\left(z-z^{\prime}\right)^{2}=d .
$$

It is easy to see that the graph $B_{n}$ has $\frac{1}{2} n^{5 / 3}+O\left(n^{4 / 3}\right)$ edges. Given a vertex $\left(x^{\prime}, y^{\prime}, z^{\prime}\right)$, the equation (3.14) has $p^{2}-p$ solutions by a theorem of Lebesgue. Thus $\left(x^{\prime}, y^{\prime}, z^{\prime}\right)$ has this many neighbors.

We claim that $B_{n}$ does not contain $K_{3,3}$. The geometric idea behind Construction 3.5 (concerning $C_{4}$-free graphs) was to join a point of the finite 
plane to the points of its "polar" (with respect to the unit circle), and then to use the fact that two lines intersect in at most one point. In contrast, the Brown construction uses the fact that, if points of the Euclidean space $\mathbb{E}^{3}$ at distance 1 are joined, then the resulting infinite graph $G$ does not contain $K_{3,3}$. This is easily seen as follows: suppose $G$ does contain $K_{3,3}$. Then the three points of one color class cannot be collinear since no point is equidistant from three collinear points. On the other hand, only two points are equidistant from three points on a circle, and so $K_{3,3}$ cannot occur. There is one problem with this "proof": in finite fields $\sum_{i} x_{i}^{2}=0$ can occur even if not all $x_{i}$ 's are 0's. Therefore in finite geometries, in some cases, a sphere can contain a whole line. So here the geometric language must be translated into the language of analytic geometry, and the right hand side of (3.14) (that is $d$ ) must be chosen appropriately.

Theorem 3.21 (Nikiforov, [198]). For $b \geq a \geq 2$ let $k \in[0, a-2]$ be an integer. Then

$$
Z(m, n, a, b) \leq(b-k-1)^{1 / a} m n^{1-(1 / a)}+(a-1) n^{1+(k / a)}+k m .
$$

For $k=0$ we get back Theorem 2.22, and substituting $k=a-2$ we obtain (3.11). Nikiforov remarks that letting $k$ run from 0 to $a-2$, we may get the best results for various values of $k$ as the relation of $m$ and $n$ varies, but we still have no constructions to substantiate this. Nikiforov also proves results on the spectral radius.

\subsection{Further applications of Algebraic Methods}

Most of the constructions providing sufficiently good lower bounds for Bipartite Extremal Graph Problems are coming either from Geometry or from Algebra21. In all these cases the vertices of the graph-construction are "coordinatized" and two vertices are joined if some (usually polynomial) equations are satisfied 22

Actually, this motivated Conjecture [1.6 or its weakening: If we use a typical finite geometric construction, then there is a $d$-dimensional space, where each vertex is joined to a $t$-dimensional subspace. Hence $n=p^{d}$, the degrees are around $n^{t / d}$, so the construction has around $n^{1+(t / d)}$ edges. The

\footnotetext{
${ }^{21}$ The Random Graph Methods are very nice but mostly they are too weak to provide sufficiently sharp lower bounds.

${ }^{22}$ Some of the constructions may seem number theoretic.
} 
conjecture suggests that there are always such almost extremal constructions 23

The most important question in this part is if one can find construction 24 to provide lower bounds where the exponents match the exponents in the upper bounds. Here we shall discuss when do we know the sharpness of the Kővári-T. Sós-Turán upper bound, $\mathbf{e x}\left(n, K_{a, b}\right)=O\left(n^{2-(1 / a)}\right)$.

As we have mentioned in Section 1.1. Kollár, Rónyai and T. Szabó [159] gave a construction which was improved by Alon, Rónyai and Szabó [11] (Constructions 3.23 and 3.25 below). The basic idea of their proofs was - at least in our interpretation - the same as that of William G. Brown; however, much more advanced. In the three dimensional Euclidean space $\mathbb{E}^{3}$ the Unit Distance Graph contains no $K_{3,3}$. If we change the underlying field to a finite field $G F(q)$ (as Brown did in Construction 3.20) then we obtain a finite graph having $n=q^{3}$ vertices. The neighborhood of each vertex will have $\approx q^{2}$ neighbors, and therefore $\approx \frac{1}{2} n^{5 / 3}$ edges. Now comes the crucial part: despite the fact, that this is highly nontrivial, we could say, that because of the geometric reason, - this graph contains no $K_{3,3}$ proving the sharpness of (2.6) 25

If we wish to extend the above construction to get lower bounds for $\operatorname{ex}\left(n, K_{a, a}\right)$ and we mechanically try to use unit balls in the $a$-dimensional space $G F(q)^{a}$ then several problems occur. We would need that any $a$ of them intersect in at most $a-1$ points. Then we would be home.

In $\mathbb{E}^{4}$ we can choose two orthogonal circles of radii $\frac{1}{\sqrt{2}}$, e.g.,

$$
\left\{\left(x_{1}, x_{2}, 0,0\right): x_{1}^{2}+x_{2}^{2}=1 / 2\right\} \text { and }\left\{\left(0,0, x_{3}, x_{4}\right): x_{3}^{2}+x_{4}^{2}=1 / 2\right\},
$$

then each point on the first one has distance 1 from each point in the second one. Hence the "Unit Distance Graph" contains $K(\infty, \infty)$. (Similarly, the "Unit Distance Graph" of $G F(q)^{4}$ contains a $K_{q, q}$.) So everything seems (!) to break down? Not quite, by the Kollár-Rónyai-Szabó construction. Instead of the 'Euclidean metric' they use a so-called norm in the space $G F\left(q^{a}\right)$. Two vectors $\mathbf{x}$ and $\mathbf{y}$ are connected if the norm of their sum is $1 ; N(\mathbf{x}+\mathbf{y})=1$. (In this context there is not much difference between

\footnotetext{
${ }^{23}$ Here we have to make some remarks about our "Conjectures": Many of them have the feature that it is not that interesting if they are true or false: in proving any alternative, we get new, important knowledge about our topics. The first such "Conjecture" was that of Turán on "Diagonal" Ramsey Numbers, that lead to the Erdös Random Graph Approach, see Remark 2.29 .

${ }^{24}$ Or "random constructions".

${ }^{25}$ Actually, as we have already discussed this in Subsection 3.20 the Will Brown's lower bound also proves this sharpness, only, the lower bound of [1] is a little better.
} 
connecting them this way or take a bipartite graph and connecting the vertices in it if $N(\mathbf{x}-\mathbf{y})=1)$.

Theorem 3.22 (Kollár, Rónyai, and T. Szabó [159] for $b>a$ !, Alon, Rónyai, and Szabó [11] for $b>(a-1) !)$. There exists $a c_{a}>0$ such that for $b>$ $(a-1)$ ! we have

$$
\operatorname{ex}\left(n, K_{a, b}\right)>c_{a} n^{2-(1 / a)} .
$$

Below we provide the Kollár-Rónyai-Szabó construction and a short verification. The norm of an element $\mathbf{x} \in G F\left(q^{a}\right)$ is defined as

$$
N(\mathbf{x}):=\mathbf{x} \cdot \mathbf{x}^{q} \cdots \mathbf{x}^{q^{a-1}} .
$$

Construction 3.23 (Kollár-Rónyai-T. Szabó [159], the Norm Graph). The vertices of $G(q, a)$ are the elements $\mathbf{x} \in G F\left(q^{a}\right)$. The elements $\mathbf{x}$ and $\mathbf{y}$ are joined if $N(\mathbf{x}+\mathbf{y})=1$.

We claim that $G(q, a)$ is $K_{a, b}$-free where $b=a !+1$. If we have a $K_{b, a} \subseteq$ $G(q, a)$, then fixing - as parameters - the $a$ vertices $\mathbf{y}_{1}, \ldots, \mathbf{y}_{a}$, we get $a$ equations of the form $N\left(\mathbf{x}+\mathbf{y}_{i}\right)=1$ with $b$ solutions $\mathbf{x} \in\left\{\mathbf{x}_{1}, \ldots, \mathbf{x}_{b}\right\}$. Then we can use the following result from Algebraic Geometry with $t=a$.

Lemma 3.24. Let $K$ be a field and $\alpha_{i, j}, \beta_{i} \in K$ for $1 \leq i, j \leq t$ such that $\alpha_{i_{1}, j} \neq \alpha_{i_{2}, j}$ if $i_{1} \neq i_{2}$. Then the system of equation

$$
\begin{array}{ccc}
\left(x_{1}-\alpha_{1,1}\right)\left(x_{2}-\alpha_{1,2}\right) \ldots\left(x_{t}-\alpha_{1, t}\right) & =\beta_{1} \\
\left(x_{2}-\alpha_{2,1}\right)\left(x_{2}-\alpha_{2,2}\right) \ldots\left(x_{t}-\alpha_{2, t}\right)= & \beta_{2} \\
\vdots & \vdots \\
\left(x_{t}-\alpha_{t, 1}\right)\left(x_{2}-\alpha_{t, 2}\right) \ldots\left(x_{t}-\alpha_{t, t}\right)= & \beta_{t}
\end{array}
$$

has at most $t$ ! solutions $\left(x_{1}, x_{2}, \ldots, x_{t}\right) \in K^{t}$.

Construction 3.25 (Alon-Rónyai-T. Szabó [1]). The vertices of the graph $H(q, a)$ are the elements $(x, X) \in G F(q)^{*} \times G F\left(q^{a-1}\right)$ and $(x, X)$ and $(y, Y)$ are joined if $N(X+Y)=x y$.

Here the norm $N(X)$ is defined in $G F\left(q^{a-1}\right)$ and so it is $X \cdot X^{q} \cdots X^{q^{a-2}}$. The graph $H(q, a)$ has $(q-1) q^{a-1}$ vertices, it is $q^{a-1}-1$ regular, and contains no $K_{a, b}$ with $b=(a-1) !+1$. To show this we use Lemma 3.24 with $t=a-1$ only.

Theorem 3.26 (Ball and Pepe [19]). The Alon-Rónyai-T. Szabó graph $H(q, 4)$ does not contain $K_{5,5}$. Hence $\mathbf{e x}\left(n, K_{5,5}\right) \geq\left(\frac{1}{2}+o(1)\right) n^{7 / 4}$. 
This is better than the earlier lower bounds of $\mathbf{e x}\left(n, K_{a, b}\right)$ for $a=5$, $5 \leq b \leq 12$, and $a=6,6 \leq b \leq 8$.

Recently, Blagojević, Bukh, and Karasev 24] gave a new algebraic construction to provide lower bounds on $Z(m, n, a, b)$ matching the (3.1) upper bound. Their example is weaker than the Kollár-Rónyai-Szabó in the sense that it only works for $b>\left(a^{2}(a+1)\right)^{a}$. On the other hand, they give new insight about the limits of the Algebraic Geometric method on which constructions may and which may not work.

We close this section mentioning that Noga Alon has a survey paper in the Handbook of Combinatorics [7] providing ample information on the topics treated here (i.e., applications of algebra in combinatorics).

\subsection{The coefficient in the Kővári-T. Sós-Turán bound}

Alon, Rónyai and Szabó [11] observed that their Construction 3.25 can be factored with a $t$-element subgroup $H \subset G F(q)^{*}$ (when $t$ divides $q-1$ ) in the same way as it was done in Construction 3.15. Namely, the vertex set of the new graph $H^{t}(q, a)$ are the elements $(x, X) \in G F(q)^{*} / H \times G F\left(q^{a-1}\right)$ and $(x, X)$ and $(y, Y)$ are joined if $N(X+Y) x^{-1} y^{-1} \in H$. Then the graph $H^{t}(q, a)$ has $n=(q-1) q^{a-1} / t$ vertices, its degrees are about $q^{a-1}$, and it contains no $K_{a, b}$ for $b=(a-1) ! t^{a-1}+1$. Let $q \rightarrow \infty$. Then also $n \rightarrow \infty$, and we get that for these fixed values of $a$ and $b$ one gets

$$
\operatorname{ex}\left(n, K_{a, b}\right) \geq(1-o(1)) \frac{\sqrt[a]{b-1}}{2 \sqrt[a]{(a-1) !}} n^{2-(1 / a)} .
$$

This shows that the order of magnitude of the coefficient in the KST bound (3.1) should be indeed $\sqrt[a]{b-1}$.

Montágh [193] found a clever factorization of the Brown graph (using the spherical symmetry of the balls) thus proving the same result with even a slightly better bound than the bound of Alon, Rónyai and Szabó, for $a=3$.

\subsection{Excluding large complete subgraphs}

The following theorem was discovered many times because its connections with Computer Science problems: given a graph $G$ with $n$ vertices, there exists a decomposition of its edges into complete balanced bipartite graphs $K_{a_{i}, a_{i}}$ having altogether $O\left(n^{2} / \log n\right)$ vertices, $\sum_{i} a_{i}=O\left(n^{2} / \log n\right)$. Lately, Mubayi and Gy. Turán [195] gave a a polynomial algorithms finding such a subgraph partition efficiently. Strictly speaking, this is not a Turán type 
problem but their result implies, e.g., that there exists a polynomial algorithm to find a $K_{a, a}$ in a graph of $n^{2} / 4$ edges of size $a=\Theta(\log n)$. The bound $O(\log n)$ is the best possible (shown by the random graph).

It is also not very difficult to show that usually the random graph gives the correct order of the Turán number $\operatorname{ex}\left(n, K_{a, a}\right)$ for $n, a \rightarrow \infty$ simultaneously.

The case when $a, b$ are very large i.e. $a+b=\Omega(n)$ was considered by Griggs, Quyang, and Ho [126], [125]. In this case $Z(m, n, a, b)$ is almost $m n$ so they considered the dual question. Let us mention only one result of this type by Balbuena, García-Vázquez, Marcote, and Valenzuela, who have more papers on this topic.

Theorem 3.27 (see [14, [16] and the references there). $Z(m, n ; a, b)=$ $m n-(m+n-a-b+1)$ if $\max \{m, n\} \leq a+b-1$.

There is another direction of research, when the ratio of $m$ and $n$ is extreme. Here we only mention a classical result, that it is easy to solve the case when $n$ is very large compared to $m$.

Theorem 3.28 (Čulík [55]).

$$
Z(m, n, a, b)=(a-1) n+(b-1)\left(\begin{array}{c}
m \\
a
\end{array}\right) \quad \text { for } \quad n \geq(b-1)\left(\begin{array}{c}
m \\
a
\end{array}\right) .
$$

\section{Excluding Cycles : $C_{2 k}$}

To start with, Bondy wrote a long chapter in the Handbook of Combinatorics [30] and also a very nice survey on Erdös and the cycles of graphs [31].

Let $\mathcal{C}$ be a (finite or infinite) set of cycles. The study of $\mathbf{e x}(n, \mathcal{C})$ is especially interesting if $\mathcal{C}$ has a member of even length. However, constructions of dense graphs without some given even cycles is usually very difficult; the examples use polarities of finite geometries (generalized polygons [171]), or Ramanujan graphs [190, [181] or some other families of polynomials [170.

An odd cycle, $C_{2 k+1}$ is chromatically critical. Hence a theorem of Simonovits 221] implies that $\operatorname{ex}\left(n, C_{2 k+1}\right)=\left[\frac{1}{4} n^{2}\right]$ for $n>n_{k}$ and the only extremal graph is $K_{\lfloor n / 2\rfloor,\lceil n / 2\rceil}$.

In this Section we concentrate on even cycles $C_{2 k}$.

\subsection{Girth and Turán numbers, upper bounds}

What is $\operatorname{ex}\left(n,\left\{C_{3}, C_{4}, \ldots, C_{g-1}\right\}\right)$, the maximum number of edges in a graph with $n$ vertices and girth $g$ ? This problem can be considered in a dual form, 
what is the least number of vertices $n=n(d, g)$ in a graph of girth $g$ and an average degree at least $d$ ? If we replace 'average' with 'minimum' $\delta$ then a simple argument gives the so-called Moore bound for odd girth:

$$
|V(G)|=n \geq n_{0}(\delta, 2 k+1):=1+\delta \sum_{0 \leq i \leq k-1}(\delta-1)^{i} .
$$

Alon, Hoory and Linial [8] showed that (4.1) holds for the average degree, too. Rearranging we have $d_{\text {ave }}<n^{1 / k}+1$, in other words

Theorem 4.1 (Upper bound when the girth is odd).

$$
\operatorname{ex}\left(n,\left\{C_{3}, C_{4}, \ldots, C_{2 k}\right\}\right)<\frac{1}{2} n^{1+(1 / k)}+\frac{1}{2} n .
$$

To prove an upper bound $n^{1+(1 / k)}$ is trivial by induction on $n$. Then (4.2) was improved but with a larger linear additive term.

Theorem 4.2 (Lam and Versträete [166], Excluding only even cycles).

$$
\operatorname{ex}\left(n,\left\{C_{4}, C_{6}, \ldots, C_{2 k}\right\}\right)<\frac{1}{2} n^{1+(1 / k)}+2^{k^{2}} n .
$$

They also note that for $k=2,3,5$ the $n$-vertex polarity graphs of generalized $(k+1)$-gons (defined by Lazebnik, Ustimenko and Woldar [171] described below as Construction 4.27) have $\frac{1}{2} n^{1+(1 / k)}+O(n)$ edges and have no even cycles of length at most $2 k$.

Corollary 4.3 ([166] and [171] Even girth is 6, 8 or 12). In case of $2 k \in\{4,6,10\}$ we have

$$
\operatorname{ex}\left(n,\left\{C_{4}, C_{6}, \ldots, C_{2 k}\right\}\right)=(1+o(1)) \frac{1}{2} n^{1+(1 / k)} .
$$

On the other hand, Füredi, Naor and Verstaëte [117] showed that if we exclude only $C_{2 k}$, then $\mathbf{e x}\left(n, C_{6}\right)>0.53 n^{4 / 3}$ (see below as Construction4.29) and Lazebnik, Ustimenko, and Woldar [169], showed that $\operatorname{ex}\left(n, C_{10}\right)>$ $0.57 n^{6 / 5}$ (see below as Construction 4.28).

Concerning the Moore bound for even girth we have

$$
|V(G)|=n \geq n_{0}(\delta, 2 k+2):=2 \sum_{0 \leq i \leq k}(\delta-1)^{i} .
$$

Alon, Hoory and Linial [8] showed that (4.5) holds for the average degree, too. Rearranging, we have $d_{\text {ave }}<(n / 2)^{1 / k}+1$, in other words 
Theorem 4.4 (Upper bound when the girth is even).

$$
\operatorname{ex}\left(n,\left\{C_{3}, C_{4}, \ldots, C_{2 k+1}\right\}\right)<\frac{1}{2^{1+(1 / k)}} n^{1+(1 / k)}+\frac{1}{2} n .
$$

This upper bound with a weaker error term was also proved earlier by Erdös and Simonovits 93.

Note that because of Theorems 3.2 , 4.21, and 4.23 one can easily show that asymptotic bound holds in (4.6) for $2 k=4,6,10$. The other cases are unsolved.

Theorem 4.5. For $2 k=4,6$ and 10 as $n \rightarrow \infty$ we have

$$
\operatorname{ex}\left(n,\left\{C_{3}, C_{4}, \ldots, C_{2 k+1}\right\}\right)=(1+o(1)) \frac{1}{2^{1+(1 / k)}} n^{1+(1 / k)} .
$$

Moreover, infinitely many exact values are obtained for $2 k=4,6,10$ : for $n=2\left(q^{k}+q^{k-1}+\cdots+q+1\right)$,

$$
\operatorname{ex}\left(n,\left\{C_{3}, C_{4}, \ldots, C_{2 k+1}\right\}\right)=(q+1)\left(q^{k}+q^{k-1}+\cdots+q+1\right)
$$

whenever $q$ is a power of a prime.

\subsection{Excluding a single $C_{2 k}$, upper bounds}

Concerning our central problem, Erdős showed that excluding just one even cycle has essentially the same effect as excluding all smaller cycles as well. This is far from trivial! Erdős never published a proof of his result.

Theorem 4.6 (Erdős, The Even Cycle Theorem).

$$
\operatorname{ex}\left(n, C_{2 k}\right)=O\left(n^{1+(1 / k)}\right) .
$$

The first proof was published by Bondy and Simonovits in the following stronger form.

Theorem 4.7 (Bondy and Simonovits [32). Let $G_{n}$ be a graph with e edges, and let $t$ satisfy $2 \leq t \leq e /(100 n)$ and $t n^{1 / t} \leq e /(10 n)$. Then $G_{n}$ contains a $C_{2 t}$.

In some sense, this is a "pancyclic theorem": there is a meta-principle, that if some reasonable conditions ensure the existence of a Hamiltonian cycle, then they ensure the existence of all shorter cycles. Here we go the other direction: if we ensure the existence of a $C_{2 k}$, then we ensure the existence of all longer cycles, up to a natural limit, with the natural parity. 
Corollary 4.8. If $G_{n}$ has at least $100 \mathrm{kn}^{1+(1 / k)}$ edges, then it contains a $C_{2 t}$, for every $t \in\left[k, k n^{1 / k}\right]$.

The Erdös-Bondy-Simonovits upper bound together with earlier known constructions imply that the exponent $1+(1 / k)$ is sharp for $C_{4}$ (see, e.g., Theorem 3.4), $C_{6}$, and $C_{10}$ (Theorems 4.22 and 4.24 below).

Corollary 4.9 (The only known exact exponents for single cycles).

$\operatorname{ex}\left(n, C_{4}\right)=\Theta\left(n^{3 / 2}\right), \quad \operatorname{ex}\left(n, C_{6}\right)=\Theta\left(n^{4 / 3}\right), \quad \operatorname{ex}\left(n, C_{10}\right)=\Theta\left(n^{6 / 5}\right)$.

The upper end of the interval in Corollary 4.8 is also sharp, apart from the constant 100 take the disjoint union of complete graphs of order $200 \mathrm{kn}^{1 / k}$. We made the following conjecture:

Conjecture 4.10 (Erdős-Simonovits). $\operatorname{ex}\left(n, C_{2 k}\right) \geq c_{k} n^{1+(1 / k)}$. Moreover,

$$
\frac{\operatorname{ex}\left(n, C_{2 k}\right)}{n^{1+(1 / k)}}
$$

converges to a positive limit.

It is only known for $C_{4}$. A weakening of this conjecture would be the following: Let $\Theta_{k, \ell}$ denote the graph of order $2+(k-1) \ell$ in which two vertices are joined by $\ell$ paths of length $k$.

Conjecture 4.11 (Simonovits). For each $k$ there is an $\ell=\ell(k)$ for which $\operatorname{ex}\left(n, \Theta_{k, \ell}\right) \geq c_{k} n^{1+(1 / k)}$.

Perhaps the first very annoying unsolved problem on this area is

Conjecture 4.12. $\operatorname{ex}\left(n, C_{8}\right) \geq c_{4} n^{5 / 4}$.

Returning to the Turán number of $C_{2 k}$, the multiplicative constant of the upper bound in the Bondy-Simonovits theorem was improved by Verstraëte 246] from 100 to 8. The best known upper bound today is that of Oleg Pikhurko:

Theorem 4.13 (Pikhurko, 203]).

$$
\operatorname{ex}\left(n, C_{2 k}\right) \leq(k-1) n^{1+(1 / k)}+16(k-1) n .
$$

\section{Historical Remark 1.}

(a) Pikhurko, in his very nice paper 203] gives a short description of the whole story.

(b) Pikhurko mentions that the Bondy-Simonovits proof gives a constant 20: originally it was stated as 100. It would be extremely interesting if the upper bound $k-1+o(1)$ for $\mathbf{e x}\left(n, C_{2 k}\right) / n^{1+(1 / k)}$ could be improved to $o(k)$. 


\subsection{Eliminating short cycles, a promising attempt}

It was relatively easy to prove the upper bound (4.2) for the number of edges for a graph $G_{n}$ with girth exceeding $2 k, e\left(G_{n}\right)=O\left(n^{1+(1 / k)}\right)$. Suppose that $G$ has no $C_{2 k}$. Erdős bipartite subgraph lemma 2.14 states that there is a bipartite subgraph $H$ with $e(H) \geq \frac{1}{2} e(G)$. This way we have eliminated all the odd cycles $C_{3}, C_{5}, \ldots, C_{2 k-1}$ from $G$. It is a natural to ask whether one can eliminate other short cycles, thus obtaining an easy proof for the Erdős-Bondy-Simonovits upper bound, (4.9).

Problem 4.14. Is it true that there exists a constant $\alpha_{2 k}>0$ such that each $C_{2 k}$-free $G_{n}$ contains an $H_{n}$ with $\operatorname{girth}\left(H_{n}\right)>2 k$ and $e\left(H_{n}\right)>\alpha_{2 k} e\left(G_{n}\right)$ ?

The answer is still unknown. The first step was done by E. Györi. The following lemma implies that $\alpha_{6}$ exists and it is at least $1 / 4$.

Lemma 4.15 (Györi [134]). If $G_{n}$ is bipartite and it does not contain any $C_{6}$, then it contains an $H_{n}$ with

$$
e\left(H_{n}\right) \geq \frac{1}{2} e\left(G_{n}\right)+1
$$

not containing $C_{4}$ 's either (for $e(G) \geq 2$ ). This is sharp only for $G_{n}=$ $K_{2, n-2}$.

We mention two generalizations.

Theorem 4.16 (Füredi, Naor and Verstraete [117]). Let $G$ be a hexagonfree graph. Then there exists a subgraph of $G$ of girth at least five, containing at least half the edges of $G$.

Furthermore, equality holds if and only if $G$ is a union of edge-disjoint complete graphs of order four or five. We got $\alpha_{6}=1 / 2$.

Theorem 4.17 (Getting rid of $C_{4}$ 's, Kühn and Osthus [165]). Every bipartite $C_{2 k}$-free graph $G$ contains a $C_{4}$-free subgraph $H$ with $e(H) \geq e(G) /(k-$ $1)$.

The factor $1 /(k-1)$ is best possible, as the example $K_{k-1, n-k+1}$ shows.

These theorems settle some special cases (namely $\mathcal{L}=\left\{C_{4}, C_{2 k}\right\}$ ) of the following compactness conjecture of Erdős and Simonovits.

Conjecture 4.18 (Compactness. Erdős-Simonovits [93]). For every finite family of graphs $\mathcal{L}$ (containing bipartite members as well) there exists an $L_{0} \in \mathcal{L}$ for which $\operatorname{ex}(n, \mathcal{L})=O\left(\operatorname{ex}\left(n, L_{0}\right)\right)$. 
The following result of Kühn and Osthus makes a little step toward solving Problem 4.14 and Conjecture 4.18.

Theorem 4.19 ([165]). Let $g \geq 4$ be an even integer and let $\ell(g)=$ : $\Pi_{1 \leq i \leq g / 2} i$. Suppose that $k-1$ is divisible by $\ell(g)$ and $G_{n}$ is a $C_{2 k}$-free graph. Then $G_{n}$ contains an $H_{n}$ with $\operatorname{girth}\left(H_{n}\right)>g$ such that $e\left(H_{n}\right) \geq$ $e\left(G_{n}\right) / 2(4 k)^{(g-2) / 2}$.

In other words, for some very special values of $k$ 's a $C_{2 k}$-free graph contains a subgraph having a positive fraction of the edges and of girth at least $\Omega(\log k / \log \log k)$.

\subsection{A lower bound for $C_{6}$ : The Benson Construction}

In the preceding section, we asserted that the Erdös theorem on even circuits is sharp for $C_{4}, C_{6}$ and $C_{10}$ (and is conjectured to be sharp in all cases). For $C_{4}$, the sharpness follows from Construction 3.5. For $C_{6}$, it can be deduced from the Benson construction [22] which we explain below. Note that (about the same time) Singleton [231] described the same graph but his definition was much more complicated.

The points of the $d$-dimensional finite projective geometry $P G(d, q)$ are the equivalence classes of the nonzero vectors of $G F(q)^{d+1}$ where $\mathbf{x}$ and $\mathbf{y}$ are equivalent if there is a $\gamma \in G F(q)^{*}$ such that $\mathbf{x}=\gamma \mathbf{y}$. There are $\left(q^{d+1}-\right.$ $1) /(q-1)$ such classes. Then the $i$-dimensional subplanes are generated by the $(i+1)$-dimensional subspaces of the vector space $G F(q)^{d+1}$.

Let

$$
A=\left(\begin{array}{lllll}
0 & 0 & 0 & 0 & 1 \\
0 & 0 & 0 & 1 & 0 \\
0 & 0 & 1 & 0 & 0 \\
0 & 1 & 0 & 0 & 0 \\
1 & 0 & 0 & 0 & 0
\end{array}\right)
$$

Clearly, $A$ is non-singular. Define the surface $S$ by the equation $\mathbf{x} A \mathbf{x}^{T}=0$,

$$
S:=\left\{\mathbf{x} \in P G(4, q): \mathbf{x} A \mathbf{x}^{T}=0\right\}
$$

Construction 4.20 (Benson's $C_{6}$-free bipartite graph). Let $\mathcal{L}$ be the set of lines of $P G(4, q)$ contained entirely in $S$. The vertex set of the bipartite graph $B_{q}$ is $S \cup \mathcal{L}$, and $\mathbf{x} \in S$ is joined to $L \in \mathcal{L}$ if $\mathbf{x} \in L$.

Theorem 4.21 (Benson [22], Singleton [231]). $B_{q}$ is a $(q+1)$-regular, bipartite, girth 8 graph with $2\left(q^{3}+q^{2}+q+1\right)$ vertices. 
Corollary 4.22. $\operatorname{ex}\left(n, C_{6}\right) \geq(1+o(1))(n / 2)^{4 / 3}$.

First, we can see that $S$ does not contain a full 2-dimensional projective plane. We can use the fact that for $\mathbf{x}$ and $\mathbf{y}$ on $S$, the line $\mathbf{x y}$ consists of the points $\mathbf{z}=a \mathbf{x}+(1-a) \mathbf{y}$, and lies entirely in $S$ if both $\mathbf{y} A \mathbf{y}^{T}=0$ and $\mathbf{x} A \mathbf{y}^{T}=0$.

Second, the number of lines from $\mathcal{L}$ containing a given point $\mathrm{x} \in S$ is $q+1$. Since the number of points on a line is $q+1$ we immediately get that $|S|=|\mathcal{L}|$.

Furthermore, $B_{q}$ contains no cycles of length $3,4,5$ or 7 . (For the odd cases this is because it is bipartite, and the existence of a 4-cycle would imply that two points of $S$ are on two distinct lines.) Now suppose that $B_{q}$ contains a 6 -cycle $v_{1} w_{1} v_{2} w_{2} v_{3} w_{3} v_{1}$. Then $S$ must contain the three lines $v_{1} v_{2}, v_{2} v_{3}$, and $v_{3} v_{1}$, and so it must contain the plane $\left\langle v_{1} v_{2} v_{3}>\right.$. But this is impossible. If we apply a coordinate transformation $T$ with $v_{1}, v_{2}$ and $v_{3}$ as the first three base vectors, we get the matrix

$$
\left(\begin{array}{lllll}
0 & 0 & 0 & ? & ? \\
0 & 0 & 0 & ? & ? \\
0 & 0 & 0 & ? & ? \\
? & ? & ? & ? & ? \\
? & ? & ? & ? & ?
\end{array}\right)
$$

since $v_{i} A v_{j}^{T}=0$. But then $A^{\prime}$ cannot be regular, contradicting the regularity of $A$. Hence $B_{q}$ cannot contain $C_{6}$ either.

All these imply that $|S|=q^{3}+q^{2}+q+1$ and that every $\mathbf{x} \in S, L \in \mathcal{L}$ if $\mathbf{x} \notin L$ then there exists a unique line $L^{\prime} \in \mathcal{L}$ such that $\mathbf{x} \in L^{\prime}$ and $L \cap L^{\prime} \neq \emptyset$.

In concluding this section, we note that finite geometry constructions can also be used in hypergraph extremal problems (see [39], 40] and [220]).

\subsection{Girth 12 graphs by Benson and by Wenger}

Theorem 4.23 (Benson [22]). Let $q$ be an odd prime power. There is a $(q+1)$-regular, bipartite, girth 12 graph $B_{q}^{*}$ with $2\left(q^{5}+q^{4}+q^{3}+q^{2}+q+1\right)$ vertices.

Corollary 4.24. $\operatorname{ex}\left(n, C_{10}\right) \geq(1+o(1))(n / 2)^{6 / 5}$.

One half of the vertex set of $B_{q}^{*}$ are the points of the quadric $Q_{6}$ in $P G(6, q)$ defined by $x_{0}^{2}+x_{1} x_{-1}+x_{2} x_{-2}+x_{3} x_{-3}=0$. Its size is exactly $\left(q^{6}-1\right) /(q-1)$. Then we select a set of lines $\mathcal{L}$ contained entirely in $Q_{6}$ and covering each point of $Q_{6}$ exactly $q+1$ times. The family $\mathcal{L}$ is selected as 
follows: If $\mathbf{x} \in Q_{6}$ and $\mathbf{x}, \mathbf{y} \in L \in \mathcal{L}$ then $\mathbf{x}$ and $\mathbf{y}$ must satisfy the following six bilinear equations:

$$
x_{0} y_{i}-x_{i} y_{0}+x_{-j} y_{-k}-x_{-k} y_{-j}=0
$$

where $(i, j, k)$ is a cyclic permutation of $(1,2,3)$ or $(-1,-2,-3)$.

Construction 4.25. The bipartite graph $B_{q}^{*}$ is defined, as before, by the incidences $\mathbf{x} \in L$.

Now consider the much simpler example of Wenger.

Construction 4.26 (Wenger [247]). Let $p$ be a prime, $k=2,3$ or $5 . H_{k}(p)$ is defined as a bipartite graph with two vertex classes $\mathbf{A}$ and $\mathbf{B}$, where $|\mathbf{A}|=$ $|\mathbf{B}|=p^{k}$ and the vertices of $\mathbf{A}$ are $k$-tuples $\mathbf{a}=\left(a_{0}, a_{1}, \ldots, a_{k-1}\right) \in G F(p)^{k}$ and same for $\mathbf{b}=\left(b_{0}, b_{1}, \ldots, b_{k-1}\right) \in \mathbf{B}$. The vertices $\mathbf{a}$ and $\mathbf{b}$ are joined if

$$
b_{j} \equiv a_{j}+a_{j+1} \cdot b_{k-1}(\bmod p) \text { for } j=0,1, \ldots, k-2 .
$$

One can see that for every $\mathbf{a} \in \mathbf{A}$ each $b_{k-1}$ determines exactly one $\mathbf{b} \in \mathbf{B}$ joined to it. This easily implies that $G[\mathbf{A}, \mathbf{B}]$ is $p$-regular, with $n=2 p^{k}$ vertices and $p^{k+1}=(n / 2)^{1+(1 / k)}$ edges.

Wenger gives an elegant proof of that $H_{2}(p)$ has no $C_{4}, H_{3}(p)$ has no $C_{4}$, nor $C_{6}$. Finally, $H_{5}(p)$ contains no $C_{4}, C_{6}$ or $C_{10}$, however, it has many $C_{8}$ 's.

\subsection{Short cycles, $C_{6}$ and $C_{10}$}

The densest constructions of $2 k$-cycle-free graphs for certain small values of $k$ arise from the existence of rank two geometries called generalized $d$-gons. These may be defined as rank two geometries whose bipartite incidence graphs are regular graphs of diameter $d$ and girth $2 d$. These are known to exist when $d$ is three, four or six. This is the background of the above Constructions 4.20 and 4.25 .

Construction 4.27 (Lazebnik, Ustimenko and Woldar [171]). One can use the existence of polarities of the generalized $(k+1)$-gons to obtain dense $2 k$-cycle-free graphs when $k \in\{2,3,5\}$. In particular, for these $k$ 's

$$
\operatorname{ex}\left(n, C_{2 k}\right) \geq \frac{1}{2} n^{1+(1 / k)}+O(n)
$$

for infinitely many $n$. 
In 93 , Erdös and Simonovits formulated the following conjecture. For fixed $k$ and $n \rightarrow \infty, \operatorname{ex}\left(n, C_{2 k}\right)=\frac{1}{2} n^{1+(1 / k)}+o\left(n^{1+(1 / k)}\right)$. This holds for $C_{4}$ (Theorem 3.4), but was disproved first for $C_{10}$, then for $C_{6}$ by the following two examples.

Construction 4.28 (Lazebnik, Ustimenko and Woldar [171]). Consider a bipartite graph $G[A, B]$ of girth exceeding $2 k$. Replace each vertex of $A$ by $k-1$ new vertices with the same neighborhood. Then the new graph $G[(k-1) A, B]$ is still $C_{2 k}$-free. In particular, starting with the girth 12 bipartite graph of Theorem 4.23 (here $k=5$ ) one gets a graph of about $5 q^{5}$ vertices and about $4 q^{6}$ edges, implying

$$
\operatorname{ex}\left(n, C_{10}\right) \geq 4(n / 5)^{6 / 5}>0.5798 n^{6 / 5}
$$

for infinitely many $n$.

Since the $C_{6}$-free graph of Construction 4.27 does not have $C_{3}$ and $C_{4}$ either, doubling a random subset appropriately, one obtains a denser $C_{6}$-free graph:

Theorem 4.29 (Füredi, Naor and Versträete [117]). For infinitely many n,

$$
\operatorname{ex}\left(n, C_{6}\right)>\frac{3(\sqrt{5}-2)}{(\sqrt{5}-1)^{4 / 3}} n^{4 / 3}+O(n)>0.5338 n^{4 / 3} .
$$

They also showed that

Theorem 4.30 (Füredi, Naor and Versträete [117]). ex $\left(n, C_{6}\right) \leq \lambda n^{4 / 3}+$ $O(n)$, where $\lambda \approx 0.6271$ is the real root of $16 \lambda^{3}-4 \lambda^{2}+\lambda-3=0$.

These theorems give the best known lower and upper bounds for $\operatorname{ex}\left(n, C_{6}\right)$. The proof of Theorem 4.30 requires a statement about hexagon-free bipartite graphs, which is interesting in its own right (see de Caen and Székely [44]). Let $\operatorname{ex}\left(m, n, C_{6}\right)$ be the maximum number of edges amongst all $m$ by $n$ bipartite hexagon-free graphs. Then

Theorem 4.31 (Füredi, Naor and Verstraëte [117]). Let $m, n$ be positive integers with $n \geq m$. Then

$$
\operatorname{ex}\left(m, n, C_{6}\right)<2^{1 / 3}(m n)^{2 / 3}+10 n .
$$

Furthermore, if $n=2 m$ then as $n$ tends to infinity,

$$
\operatorname{ex}\left(m, n, C_{6}\right)= \begin{cases}2^{1 / 3}(m n)^{2 / 3}+O(n) & \text { for infinitely many } m \\ 2^{1 / 3}(m n)^{2 / 3}-o\left(n^{4 / 3}\right) & \text { for all } m .\end{cases}
$$

The lower bound is given by the graph defined in Construction 4.28 starting with the Benson graph (Theorem 4.21, $k=3$ ). 


\subsection{Bipartite hosts with extreme sides}

We have already seen two such results concerning the Zarankiewicz number, by Reiman (Theorem 3.2) and Čulík (Theorem 3.28). András Sárközy and Vera Sós formulated the following conjecture 26

\section{Conjecture 4.32.}

$$
\mathbf{e x}\left(m, n, C_{6}\right)<2 n+c(n m)^{2 / 3}
$$

A weaker version of this was proved by Gábor N. Sárközy, 212] and later Győri [134] proved a stronger

Theorem 4.33. There exists a constant $c_{k}>0$ for which if $G[A, B]$ is a bipartite graph with color classes $A, B$, and $|A|=m,|B|=n \geq m^{2}$, and

$$
e(G[A, B]) \geq(k-1) n+c_{k} m^{2},
$$

then $G[A, B] \supset C_{2 k}$.

This means that for $n>m^{2}$ the extremal number becomes linear. For more recent results see, e.g., Balbuena, García-Vázquez, Marcote, and Valenzuela [15. Later Györi [135] showed that $c_{3}=1 / 8$, proving

$$
\operatorname{ex}\left(m, n, C_{6}\right) \leq 2 n+\frac{1}{8} m^{2},
$$

for $n, m>100, n \geq m^{2} / 16$ and here equality holds if $m$ is a multiple of 4 .

\subsection{The effect of odd cycles}

Let $\mathcal{L}$ be a set of graphs and let $\operatorname{ex}_{\text {bip }}(n, \mathcal{L})$ denote the bipartite Turán number of $\mathcal{L}$, the size of the largest $\mathcal{L}$-free bipartite graph on $n$ vertices.

Theorem 4.34 (Erdős and Simonovits [93]).

$$
\operatorname{ex}\left(n,\left\{C_{4}, C_{5}\right\}\right)=(1+o(1)) \mathbf{e x}_{\mathrm{bip}}\left(n, C_{4}\right)=(1+o(1))(n / 2)^{3 / 2} .
$$

They also conjecture that the same holds for $\left\{C_{3}, C_{4}\right\}$ (i.e., for the girth problem) but this is still unsolved. Then, they make the following bold conjecture.

\footnotetext{
${ }^{26}$ A weaker version of this conjecture was formulated by Erdős several years earlier.
} 
Conjecture 4.35 (Erdős and Simonovits [93] on the effect of odd cycles).

Let $\mathcal{C}_{2 \ell+1}^{\text {odd }}$ denote the set of odd cycles $\left\{C_{3}, C_{5}, \ldots, C_{2 \ell+1}\right\}$. For any family $\mathcal{L}$ consisting of bipartite graphs there exists an odd integer $2 \ell+1$ such that $\operatorname{ex}\left(n, \mathcal{L} \cup \mathcal{C}_{2 \ell+1}^{\text {odd }}\right) \approx \operatorname{ex}_{\text {bip }}(n, \mathcal{L})$.

This conjecture was verified in a few cases by extending and sharpening Theorem 4.34 as follows.

Theorem 4.36 (Keevash, Sudakov and Verstraëte [157]). Let $\mathcal{C}_{2 k}^{\text {even }}$ denote the set of even cycles $\left\{C_{4}, C_{6}, \ldots, C_{2 k}\right\}$. Suppose that $2 k \in\{4,6,10\}$ and suppose that $2 \ell+1>2 k$. Then

$$
\operatorname{ex}\left(n, \mathcal{C}_{2 k}^{\text {even }}, C_{2 \ell+1}\right)=(1+o(1)) \operatorname{ex}_{\text {bip }}\left(n, \mathcal{C}_{2 k}^{\text {even }}\right) \sim(n / 2)^{1+(1 / k)} .
$$

They even proved a stability result (when $n \rightarrow \infty$ ) and, using it, an exact version: If $2 k \in\{4,6,10\}$ and $2 \ell+1 \geq 5,15$, or 23 , respectively, and $n=2\left(q^{k}+q^{k-1}+\cdots+q+1\right)$ then for $n>n_{2 \ell+1}$ we have

$$
\operatorname{ex}\left(n, \mathcal{C}_{2 k}^{\text {even }} \cup C_{2 \ell+1}\right) \leq(q+1) n
$$

and here equality holds only if there is a generalized $(k+1)$-gon of order $q$.

In a more recent work Allen, Keevash, Sudakov and Verstraëte [5] verified the stronger form of the Erdös-Simonovits conjecture proving that for any fixed $2 \ell+1 \geq 5$ one has $\operatorname{ex}\left(n,\left\{K_{2, t}, C_{2 \ell+1}\right\}\right) \sim \operatorname{ex}_{\text {bip }}\left(n, K_{2, t}\right)$ and $\operatorname{ex}\left(n,\left\{K_{3,3}, C_{2 \ell+1}\right\}\right) \sim \operatorname{ex}_{\text {bip }}\left(n, K_{3,3}\right)$. They also show

$$
\operatorname{ex}\left(n,\left\{K_{2, t}, B_{t}, C_{2 \ell+1}\right\}\right) \sim \operatorname{ex}_{\text {bip }}\left(n,\left\{K_{2, t}, B_{t}\right\}\right) \sim(n / 2)^{3 / 2}
$$

for any fixed $t \geq 2$ and $2 \ell+1 \geq 9$, where $B_{t}$ is a "book" of $t C_{4}$ 's sharing and edge: it has $2 t+2$ vertices and $3 t+1$ edges. Their main tool is the smoothness of the corresponding Turán number's and the sparse regularity lemma of A. Scott 213.

On the other hand, for any $t \geq 1$ and prime $q>2^{t^{4}}$, they construct $(t+2)$-partite graphs $G_{q, t}$ with no triangle or $K_{2,2 t+1}$ having $n=(t+2) q^{2}$ vertices and $\left(\begin{array}{c}t+2 \\ 2\end{array}\right) q^{2}(q-1)$ edges. This implies

$$
\operatorname{ex}\left(n,\left\{K_{2,2 t+1}, C_{3}\right\}\right) \geq(1+o(1)) \frac{t+1}{\sqrt{t+2}} n^{3 / 2} .
$$

So, using $\operatorname{ex}_{\text {bip }}\left(n, K_{2 t+1}\right) \sim \sqrt{t} n^{3 / 2}$, which follows easily from (3.7) and (3.9), they obtain

$$
\liminf _{n \rightarrow \infty} \frac{\mathbf{e x}\left(n,\left\{K_{2,2 t+1}, C_{3}\right\}\right)}{\mathbf{e x}_{\text {bip }}\left(n, K_{2,2 t+1}\right)} \geq \frac{t+1}{\sqrt{t(t+2})}>1 .
$$


In particular the ratio is $2 / \sqrt{3}+o(1)$ for $K_{2,3}$. We explain their construction yielding (4.12) only for $t=1$.

Construction 4.37 (Allen, Keevash, Sudakov and Verstraëte [5]). Let $q \equiv$ $2(\bmod 3)$ be a prime. Let $G^{q}$ be a three-partite graph with parts $A_{1}, A_{2}$ and $A_{3}$ which are copies of $G F(q) \times G F(q)$. Join $\left(x_{1}, x_{2}\right) \in A_{i}$ to $\left(y_{1}, y_{2}\right) \in A_{i+1}$ if

$$
\left(y_{1}, y_{2}\right)=\left(x_{1}, x_{2}\right)+\left(a, a^{2}\right)
$$

for some $a \in G F(q), a \neq 0$.

The obtained graph is $K_{2,3}$ and $C_{3}$-free, and has $n=3 q^{2}$ vertices and almost $n^{3 / 2} / \sqrt{3}$ edges. This yields the ratio $2 / \sqrt{3}+o(1)$ for $K_{2,3}$ in (4.13). They believe that Erdös' Conjecture 1.8 is false:

Conjecture $4.38([5])$.

$$
\liminf _{n \rightarrow \infty} \frac{\mathbf{e x}\left(n,\left\{C_{3}, C_{4}\right\}\right)}{\mathbf{e x}_{\mathrm{bip}}\left(n, C_{4}\right)}>1 .
$$

\subsection{Large girth: Ramanujan graphs}

Until this point we were fixing the excluded subgraphs. However, there is a subcategory of extremal graph problems, which we could also call "Parametrized Extremal Graph Problems". Instead of defining them we give an almost trivial but important example: Horst Sachs and Erdős [87] reformulated the Moore bounds (4.1) (4.5), in a slightly different form.

Theorem 4.39. If the minimum degree of $G_{n}, d:=d_{\min }\left(G_{n}\right)>2$ then $G_{n}$ contains $a C_{\ell}$ with

$$
\ell<\frac{2 \log n}{\log (d-1)}
$$

Here we arrived at an area where some constructions (for lower bounds) were needed, and the lower bounds were easily obtained by probabilistic arguments; however they were very difficult to obtain them in a constructive way. Instead of going into details, we mention a result of Margulis [188] that (4.14) is sharp up to a constant: there are - not too complicated - Cayley graphs of constant (even) degrees $d$ and girth at least $c \log _{d-1} n$. Here surprisingly, Margulis' construction is better than the random graph and a construction of Imrich yields an even better constant $c$ : 
Theorem 4.40 (Imrich [147]). For every integer $d>2$ one can (effectively) construct infinitely many d-regular Cayley graphs $X_{n}$ with

$$
\operatorname{girth}\left(X_{n}\right)>0.4801 \frac{\log n}{\log (d-1)}-2 .
$$

The next step in this area was a much deeper and more important results of Margulis [190, 189], Lubotzky, Phillips and Sarnak, [181] on the Expander graphs, that are eigenvalue-extremal. In this sense the Margulis-LubotzkyPhillips-Sarnak graph is very nice. There is only one problem with it. While defining these graphs is non-trivial, but not extremely complicated, to verify their extremal properties requires deep mathematical tools. Below we give a very compressed description of it.

Definition 4.41. Given a connected $k$-regular graph $X$, we denote by $\lambda(X)$ the largest of the absolute values of eigenvalues of the adjacency matrix of $X$, different from $k$. An $n$-vertex $k$-regular graph $X_{n, k}$ is a Ramanujan graph if $\lambda\left(X_{n, k}\right) \leq 2 \sqrt{k-1}$.

Remark 4.42. In case of $k$-regular graphs, the largest absolute values of the eigenvalues is $k$. The bipartite graphs have the property that if $\lambda_{i}$ is eigenvalue, then $-\lambda_{i}$ is also an eigenvalue. By the Alon-Boppana inequality, (see Proposition 4.2 of [181])

$$
\liminf _{n \rightarrow \infty} \lambda\left(X_{n, k}\right)=2 \sqrt{k-1}
$$

Ramanujan graphs are important because they are expander graphs, which are extremely important in Theoretical Computer Science.

There are quite a few cases, where - instead of using "random graph constructions" one tries to use Cayley Graphs. Cayley graphs are graphs whose vertices are the elements of some group $\mathcal{G}$ and the edges are the pairs $\left(g, \alpha_{i} g\right)$, where $g \in \mathcal{G}$ and $\alpha_{1}, \ldots, \alpha_{k}$ are some elements of $\mathcal{G}$. If we look for a digraph, then this is a correct definition. However, if we are looking for ordinary graphs, then we have to assume that $S:=\left\{\alpha_{1}, \ldots, \alpha_{k}\right\}$ is closed under taking the inverse: if $\alpha \in S$ then $\alpha^{-1} \in S$ as well. If we choose $\mathcal{G}$ and $S$ appropriately, then the obtained graph will provide us with nice constructions; mainly, because it behaves as if it were a random graph, or, occasionally, even better.

Construction 4.43 ([181]). Let $p$ and $q$ be unequal primes congruent to 1 mod 4. The Ramanujan graphs $X^{p, q}$ of [181] are $p+1$-regular Cayley 
graph 27 of the group $\mathbf{P S L}(2, \mathbb{Z} / q \mathbb{Z}): p+1$ generators of the group are fixed, which are obtained from the solutions of

$$
p=a^{2}+b^{2}+c^{2}+d^{2} \text {, where } a>0 \text { is odd and } b, c, d \text { are even. }
$$

The number of solutions of (4.15) is connected to the famous Ramanujan conjecture, which is still open. However, good approximations are known, by Eichler and Igusa, enough for the purposes of [181]. Originally most of the authors were interested in the eigenvalue properties (spectral gap) of these graphs, that are also strongly connected to them being expander graphs (see Alon, 6], Alon-Milman [10]).

From here on, $X_{n, k}=X^{p, q}$ is a special sequence of Ramanujan graphs, which is non-bipartite if the Jacobi symbol $\left(\frac{q}{p}\right)=1$; then it has $n=\left(q^{3}-q\right) / 2$ vertices.

Theorem 4.44. For $k=p+1, X^{p, q}$ is $k$-regular, its eigenvalues are $\lambda= \pm k$ or $|\lambda| \leq 2 \sqrt{k-1}$.

This property is optimal and leads to the best known explicit expander graphs. Alon turned the attention of the authors to that these graphs satisfy a number of extremal combinatorial properties.

Theorem 4.45 (Observation of Alon). The girth of $X_{n, k}$ is asymptotically $\geq \frac{4}{3} \frac{\log n}{\log (k-1)}$.

This gives larger girth than what was previously known by explicit or non-explicit constructions. Also, it is one of the "cleanest" way to define graphs with large girth and high chromatic number:

Theorem 4.46 ([181]). If $X_{n, k}$ is a non-bipartite Ramanujan graph, then its independence number and chromatic number satisfy

$$
\alpha\left(X_{n, k}\right) \leq \frac{2 \sqrt{k-1}}{k} n \quad \text { and } \quad \chi\left(X_{n, k}\right) \geq \frac{k}{2 \sqrt{k-1}}
$$

For a more informative description of these and many other related areas see the survey of Alon in the Handbook [7].

\footnotetext{
${ }^{27}$ There are two of them, a bipartite and a non-bipartite, we forget the bipartite one.
} 


\subsection{The girth problem: the Lazebnik-Ustimenko approach}

After 20 years Theorem 4.47 still yields the best known lower bound for the girth problem: Lazebnik, Ustimenko and Woldar's work [170] gives a slight improvement (an $O(1)$ in the denominator of the exponent) to what we can get from the Ramanujan' graphs.

Theorem 4.47 ([170]). $\operatorname{ex}\left(n,\left\{C_{3}, C_{4}, \ldots, C_{2 k+1}\right\}\right)=\Omega\left(n \cdot n^{2 /(3 k-3+\varepsilon)}\right)$ where $k \geq 2$ is fixed, $\varepsilon=0$ if $k$ is odd, $\varepsilon=1$ if $k$ is even and $n \rightarrow \infty$.

We have seen basically two approaches on how to construct graphs with high girth. One was the use of Finite Geometries, and the other the use of Cayley Graphs of some matrix groups (Ramanujan graphs). There is (at least) one further important approach to this question which we find in the works of Lazebnik and Ustimenko and later Lazebnik, Ustimenko and Woldar.

Remark 4.48 (History). In this survey many important areas had to be skipped. One of them is the family of Lazebnik-Ustimenko type algebraic constructions. This family of constructions is much more flexible than many earlier ones, and provides a lot of new constructions in extremal graph theory, in Ramsey type problems, for graphs and hypergraphs as well. The first results were achieved by Lazebnik and Ustimenko [168]. Lazebnik and his coworkers created a school in this area. The reader is referred here to [167].

The main feature of this approach can be described (perhaps slightly cheating) as follows. We take a set $R$ (a finite or infinite ring or field), its $d^{\text {th }}$ power, and a sequence of polynomials $f_{2}, \ldots, f_{d}$. Define a bipartite graph, where the colour classes $A$ and $B$ consist of vectors $\left(a_{1}, \ldots, a_{d}\right)$ and $\left(b_{1}, \ldots, b_{d}\right)$ that are joined if

$$
\begin{aligned}
a_{2}+b_{2} & =f_{2}\left(a_{1}, b_{1}\right) \\
a_{3}+b_{3} & =f_{3}\left(a_{1}, b_{1}, a_{2}, b_{2}\right) \\
\ldots & \\
a_{d}+b_{d} & =f_{d}\left(a_{1}, b_{1}, \ldots, a_{d}, b_{d}\right) .
\end{aligned}
$$

We may also identify $A$ and $B$ to get non-bipartite graphs as well. In general, either we get digraphs, or some symmetry conditions are assumed on the functions $f_{i}$, ensuring that if $\left(a_{1}, \ldots, a_{d}\right)$ is joined to $\left(b_{1}, \ldots, b_{d}\right)$, then $\left(b_{1}, \ldots, b_{d}\right)$ and $\left(a_{1}, \ldots, a_{d}\right)$ are joined as well. Yet, it is not an easy area to describe it on a few pages: this is why we basically skip it. Perhaps the more interested reader should look at [172]. 


\subsection{Cycle length distribution}

As a measure of the density of the cycle lengths in a graph $G$, Erdös introduced the number $L(G)$, the sum of the reciprocals of the distinct cycle lengths of $G$. The following beautiful theorem, due to Gyárfás, Komlós and Szemerédi, proves a conjecture of Erdős and Hajnal, asserting that in some sense the complete graph or the complete bipartite graph are the densest concerning cycle lengths:

Theorem 4.49 ([131]). There exists a positive constant $c>0$ such that if $d_{\min }(G) \geq k$, then for the sum of the reciprocals of the cycle lengths $\ell_{i}$ of $G$ we have

$$
L(G)=\sum \frac{1}{\ell_{i}}>c \log k .
$$

The union of complete graphs $K_{k+1}$ or bipartite graphs $K_{k, m}$ (where $m \geq k$ ) show that this lower bound is sharp.

Generalizing a theorem of Bondy and Vince 33, Gengua Fan proved several nice results on the distribution of cycle lengths. We mention only one of them.

Theorem 4.50 (G. Fan [96]). Let xy be an edge in a 2-connected graph $G$, $k$ be a positive integer and suppose that all the vertices of $G$ but $x$ and $y$ have degrees at least $3 k$. Then $x y$ is contained in $k+1$ cycles $C^{0}, C^{1}, \ldots, C^{k}$, such that $k+1<\left|E\left(C^{0}\right)\right|<\left|E\left(C^{1}\right)\right|<\cdots<\left|E\left(C^{k}\right)\right|,\left|E\left(C^{i}\right)\right|-\left|E\left(C^{i-1}\right)\right|=2$ for $i=1, \ldots, k-1$ and $1 \leq\left|E\left(C^{k}\right)\right|-\left|E\left(C^{k-1}\right)\right| \leq 2$.

A related result concerning $k$ odd cycle lengths can be found in Gyárfás [130].

Next we recall a conjecture of Burr and Erdős.

Conjecture 4.51 (Burr and Erdős). For every odd integer $k>0$, and every integer $\ell$, there exists a $c_{k}$ such that if $e\left(G_{n}\right)>c_{k} n$, then some $m \equiv \ell$ $(\bmod k)$, we have $C_{m} \subseteq G_{n}$.

This was proved by Bollobás 25] with $c_{k} \leq\left((k+1)^{k}-1\right) / k$. Häggkvist and Scott (137, 138) decreased $c_{k}$ and extended the Bollobás result, proving that every graph $G_{n}$ with minimum degree at least $300 k^{2}$ contains $k$ cycles of consecutive even lengths. Soon after, the right order of magnitude of $c_{k}$ was established.

Theorem 4.52 (Verstraëte [246]). Let $G_{n}$ be a graph with $e\left(G_{n}\right) \geq 4 k n$. Then there are cycles of $k$ consecutive even lengths in $G_{n}$.

We close this part with the following theorem: 
Füredi-Simonovits: Degenerate (bipartite) extremal graph problems

Theorem 4.53 (Sudakov, Verstraëte [234]). Let $\operatorname{girth}\left(G_{n}\right)=g$ be fixed and $d=2 e\left(G_{n}\right) / n$. Let $\mathcal{C}(G)$ denote the set of cycle-lengths in $G$. Then $\mathcal{C}\left(G_{n}\right)$ contains at least $\Omega\left(d^{\lfloor(g-1) / 2\rfloor)}\right)$ consecutive even integers, as $d \rightarrow \infty$.

\section{Paths and long cycles}

In this section we shall describe results connected with $\operatorname{ex}\left(n, P_{k}\right), \operatorname{ex}\left(n, \mathcal{C}_{\geq k}\right)$, (where the cycles of at least $k$ vertices are excluded). This problem was proposed by Turán and the (asymptotic) answer were given by Erdős-Gallai.

\subsection{Excluding long cycles}

Theorem 5.1 (Erdös and Gallai [80]). Let $G_{n}$ be a graph with more than $\frac{1}{2}(k-1)(n-1)$ edges, $k \geq 3$. Then $G_{n}$ contains a cycle of length at least $k$. This bound is the best possible if $n-1$ is divisible by $k-2$.

A matching lower bound $\frac{1}{2}(k-1) n-O\left(k^{2}\right)$ can be obtained gluing together complete graphs of sizes at most $k-1$. If $k$ is odd, then there are nearly extremal graphs having a completely different structure. Namely, one can take a complete bipartite graph with partite sets $A$ and $B$ of sizes $|A|=\frac{k-1}{2}$ and $|B|=n-\frac{k-1}{2}$ and add all edges in $A$, too.

The exact value was determined by Woodall [249] and independently and at the same time by Kopylov [162].

Theorem 5.2 ([162], 249]). Let $n=m(k-2)+r$, where $1 \leq r \leq k-2$, $k \geq 3, m \geq 1$ integers. If

$$
e\left(G_{n}\right)>m\left(\begin{array}{c}
k-1 \\
2
\end{array}\right)+\left(\begin{array}{l}
r \\
2
\end{array}\right)
$$

then $G_{n}$ contains a cycle of length at least $k$, and this bound is the best possible:

$$
\operatorname{ex}\left(n, \mathcal{C}_{\geq k}\right)=\frac{1}{2}(k-1) n-\frac{1}{2} r(k-r) .
$$

Caccetta and Vijayan [43] gave an alternative proof of the result. We need a definition.

Construction 5.3. Let $H_{n, k, s}$ be an n-vertex graph consisting of a complete graph $K_{k-s}$ on the set $A \cup B,|A|=k-2 s,|B|=s$ and a complete bipartite graph $K_{s, n-(k-s)}$ with parts $B$ and $C$ where $A, B$ and $C$ form a partition of $V(H)$ (hence $|C|=n-(k-s)$ and $n \geq k,(k-1) / 2 \geq s \geq 1)$. 
The graph $H$ contains no cycle of size $k$ or larger and for $s \geq 2$ it is 2-connected. Denote its size by $h(n, k, s)$.

They all ([162], [249], [43]) characterized the structure of the extremal graphs in Theorem 5.2, Namely either

- the blocks of $G_{n}$ are $m$ complete graphs $K_{k-1}$ and a $K_{r}$, or

- $k$ is odd, $r=(k+1) / 2$ or $(k-1) / 2$ and $q$ of the blocks of $G_{n}$ are $K_{k-1}$ 's and a copy of a $H_{n-q(k-2), k,(k-1) / 2}$.

The strongest result on the field is due to Kopylov who also investigated 2-connected graphs.

Theorem 5.4 (Kopylov [162]). Suppose that $n \geq k \geq 5$ and the 2-connected graph $G_{n}$ contains no cycles of length of $k$ or larger. Then

$$
e\left(G_{n}\right) \leq \max \left\{h(n, k, 2), h\left(n, k,\left\lfloor\frac{1}{2}(k-1)\right\rfloor\right)\right\}
$$

and this bound is the best possible.

Moreover, only the graphs $H_{n, k, s}$ could be extremal, $s \in\{2,\lfloor(k-1) / 2\rfloor\}$.

This theorem was also conjectured by Woodall [249] and he also proved it for $n \geq(3 k-5) / 2$. It was also reproved much later in 97 .

\section{2. $\quad$ Excluding $P_{k}$}

One of the oldest problems is the question of determining $\mathbf{e x}\left(n, P_{k}\right)$.

Theorem 5.5 (Erdős and Gallai [80]). If $G_{n}$ is a graph containing no $P_{k}$, $(k \geq 2)$, then

$$
e\left(G_{n}\right) \leq \frac{k-2}{2} n
$$

with equality if and only if $k-1$ divides $n$ and all connected components of $G$ are complete graphs on $k-1$ vertices.

Consider the $n$-vertex graph $G_{n}$ which is the union of $\lfloor n /(k-1)\rfloor$ vertexdisjoint $K_{k-1}$ and a $K_{r}(0 \leq r \leq k-2)$. If $T_{k}$ is any connected $k$-vertex graph, then $T_{k} \nsubseteq G_{n}$. Hence

$$
\operatorname{ex}\left(n, T_{k}\right) \geq \frac{k-2}{2} n-\frac{1}{8} k^{2} .
$$

In particular,

$$
\operatorname{ex}\left(n, P_{k}\right) \geq \frac{k-2}{2} n-\frac{1}{8} k^{2}
$$




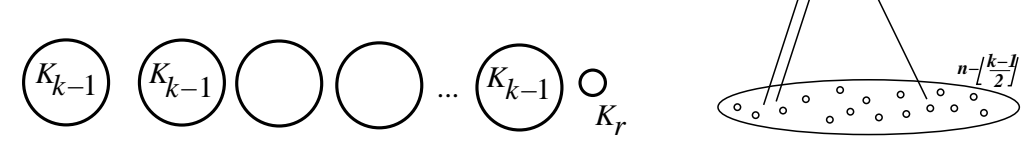

Figure 2: Potential extremal graphs

If $k$ is even, then there are nearly extremal graphs having a completely different structure. Namely, one can take a complete bipartite graph with partite sets $A$ and $B$ of sizes $|A|=\frac{k-2}{2}$ and $|B|=n-\frac{k-2}{2}$ and add all edges in $A$, too (Fig. 2). Faudree and Schelp [98, proved that the extremal graph for $P_{k}$ can indeed be obtained in this way for all $n$ and $k$. They needed this to prove some Ramsey theorems on paths. The variety of extremal graphs makes the solution difficult.

Theorem 5.6 (Faudree and Schelp [98] and independently Kopylov [162]). Let $n \equiv r(\bmod k-1), 0 \leq r<k-1, k \geq 2$. Then

$$
\operatorname{ex}\left(n, P_{k}\right)=\frac{1}{2}(k-2) n-\frac{1}{2} r(k-1-r) .
$$

Faudree and Schelp also described the extremal graphs which are either

- vertex disjoint unions of $m$ complete graphs $K_{k-1}$ and a $K_{r}$, or

- $k$ is even and $r=k / 2$ or $k / 2-1$ and another extremal graphs can be obtained by taking a vertex disjoint union of $t$ copies of $K_{k-1}(0 \leq t<m)$ and a copy of $H(n-t(k-1), k-1, k / 2-1)$.

Theorem 5.7 (Kopylov 162]). Let $G_{n}$ be a connected graph containing no $P_{k},(k \geq 4)$ and $n \geq k$. Then

$$
e(G) \leq \max \left\{h(n, k-1,1), h\left(n, k-1,\left\lfloor\frac{1}{2}(k-2)\right\rfloor\right)\right\}
$$

and this bound is the best possible.

Moreover, only the graphs $H_{n, k-1, s}$ could be extremal, $s \in\{1,\lfloor(k-$ 2) $/ 2\rfloor\}$.

Balister, Győri, Lehel and Schelp [18] also provided the extremal structures. 


\subsection{Proof ideas}

Let $\mathbf{e x}_{\mathrm{con}}\left(n, P_{k}\right)$ be the maximum number of edges in connected, $n$-vertex, $P_{k}$-free graphs, and let $\mathbf{e x}_{2-\text { con }}\left(n, \mathcal{C}_{\geq k}\right)$ denote the maximum number of edges in 2-connected, $n$-vertex, $\mathcal{C}_{\geq k}$-free graphs. Determining these functions give upper bounds for $\operatorname{ex}\left(n, P_{k}\right)$ and $\operatorname{ex}\left(n, \mathcal{C}_{\geq k}\right)$.

Indeed, every $P_{k}$-free graph is a vertex disjoint union of $P_{k}$-free components, we have

$$
\operatorname{ex}\left(n, P_{k}\right)=\max _{\sum n_{i}=n, n_{i} \geq 1} \sum \operatorname{ex}_{\mathrm{con}}\left(n_{i}, P_{k}\right)
$$

Similarly, a maximal $\mathcal{C}_{\geq k}$-free graph is connected and every connected graph is a cactus-like union of 2-connected blocks (and edges) so we have

$$
\operatorname{ex}\left(n, \mathcal{C}_{\geq k}\right)=\max _{\sum\left(n_{i}-1\right)=n-1, n_{i} \geq 2} \sum \mathbf{e x}_{2-\operatorname{con}}\left(n_{i}, \mathcal{C}_{\geq k}\right)
$$

where we define $\mathbf{e x}_{2-\operatorname{con}}\left(2, \mathcal{C}_{\geq k}\right)=1$.

Let $G$ be a connected, $n$-vertex, $P_{k}$-free graph. Add a new vertex to it and join to all other vertices. We obtain $G_{n+1}$ with $e\left(G_{n+1}\right)=e(G)+n$. This new graph has no cycle of length exceeding $k$ and its connectivity is one larger than that of $G_{n}$. We obtain

$$
\operatorname{ex}\left(n, P_{k}\right)+n \leq \mathbf{e x}\left(n+1, \mathcal{C}_{\geq k+1}\right)
$$

and

$$
\operatorname{ex}_{\mathrm{con}}\left(n, P_{k}\right)+n \leq \mathbf{e x}_{2-\operatorname{con}}\left(n+1, \mathcal{C}_{\geq k+1}\right) .
$$

So Theorem 5.1 and (5.6) imply Theorem 5.5. Similarly, Theorem 5.2 and (5.6) imply Theorem 5.6.

The upper bounds for $\mathbf{e x}_{2-\text { con }}\left(n, \mathcal{C}_{\geq k+1}\right)$ yield upper bounds for $\operatorname{ex}_{\mathrm{con}}\left(n, P_{k}\right)$. (Actually, (5.7) and Theorem 5.4 lead to the solution of $\mathbf{e x}_{\mathrm{con}}\left(n, P_{k}\right)$, Theorem 5.7).

Again Theorem 5.4 and (5.5) lead to Theorem 5.2 which is obviously stronger than Theorem 5.1 .

Finally, the proof of Theorem 5.4 uses induction on $n$ and $k$, by deleting small degree vertices, contracting edges, and finally applying Pósa's theorem on Hamiltonian graphs.

\subsection{Generalizations}

In a recent work Lidický, Hong Liu and Cory Palmer [174] determined the exact Turán number (and the unique extremal graph) when the forbidden 
graph $L$ is a linear forest, each component is a path. They also considered star-forests.

Gyárfás, Rousseau, and Schelp [132] determined $\operatorname{ex}\left(K(m, n), P_{k}\right)$ for all $m, n, k$. Their formula and proof are rather involved, they distinguish 10 subcases.

\section{Excluding trees}

Here we shall discuss two extremal problems on trees: the Erdős-Sós conjecture and the Loebl-Komlós-Sós conjecture.

\subsection{Erdős-Sós conjecture}

We have already discussed the Erdös-Gallai theorems. Since the extremal numbers for $P_{k}$ and for the star $K_{1, k-1}$ are roughly the same, this led Erdös and T. Sós to the following famous conjecture.

Conjecture 6.1 (Erdős-Sós [63]). Let $T_{k}$ be an arbitrarily fixed k-vertex tree. If a graph $G_{n}$ contains no $T_{k}$, then

$$
e\left(G_{n}\right) \leq \frac{1}{2}(k-2) n .
$$

As we have seen - by (5.2) - the disjoint union of complete graphs $K_{k-1}$ shows that $\operatorname{ex}\left(n, T_{k}\right) \geq \frac{1}{2}(k-2) n-\frac{1}{8} k^{2}$. Though several partial cases were settled, the upper bound was unknown until Ajtai, Komlós, Simonovits, and Szemerédi proved:

Theorem 6.2 (Main Theorem, Sharp [1, 2, 3]). There exists an integer $k_{0}$ such that if $k>k_{0}$ and $T_{k}$ is an arbitrarily fixed $k$-vertex tree, and the graph $G_{n}$ contains no $T_{k}$, then

$$
e\left(G_{n}\right) \leq \frac{1}{2}(k-2) n .
$$

Below we list a few subcases where this conjecture is verified, but we do not try to give a complete list.

Theorem 6.3 (Sidorenko [215]). If $T_{k}$ has a vertex $x$ connected to at least $k / 2$ vertices of degree 1 (i.e., leaves) then the Erdös-Sós conjecture holds for this $T_{k}$.

Theorem 6.4 (McLennan [182]). If the diameter of $T_{k}$ is at most 4 , then the Erdös-Sós conjecture holds for this $T_{k}$. 
Dobson (and coauthors) have several results in this area, under some strong condition of sparsity. We mention only the Brandt-Dobson theorem [34, or Sacle and Wozniak, [251], 211].

\subsection{Sketch of the proof of Theorem 6.2}

We are given a $T_{k}$, and a $G_{n}$ violating (6.2). We wish to embed $T_{k}$ into $G_{n}\left(T_{k} \hookrightarrow G_{n}\right)$. The proof is very involved and will be given in three rather long papers. The following weakening plays a central role.

Theorem 6.5 ( $\eta$-weakening [1]). For any (small) constant $\eta>0$ there exists a $k_{0}(\eta)$ such that for $n \geq k>k_{0}(\eta)$, if

$$
e\left(G_{n}\right)>\frac{1}{2}(k-2) n+\eta k n,
$$

then each $k$-vertex tree $T_{k}$ is contained in $G_{n}$.

(a) First, in [1] we prove this theorem. If, in addition, we assume that $G_{n}$ is dense: for some $c>0, k>c n$, then we can apply the Szemerédi Regularity Lemma [235]. The proof of this theorem follows basically the line which was later used to prove the Loebl Conjecture, by Ajtai, Komlós and Szemerédi [4] and later by Yi Zhao 255]. Also it was used in the KomlósSós conjecture by Diana Piguet and Maya Stein [201, Cooley [54, Hladký and Piguet [144, in stronger and stronger form, and now the publication of that proof is almost finished by Hladký, Komlós, Piguet, Simonovits, Stein, and Szemerédi [143].

(b) In the second part, 2] we prove several theorems asserting that under some very special conditions $T_{k} \subseteq G_{n}$. Some of these steps are "stability arguments".

Analyzing the proof of Theorem 6.5. shows that either we can gain at some points, in some of the estimates $\eta k n$ edges, and therefore Theorem 6.5 (more precisely, its slightly modified proof) implies the sharp version, Theorem 6.2. or else $G_{n}$ must have a very special structure: it contains a smaller copy of the conjectured extremal graphs: for some $m \approx k$,

$\left(b_{1}\right)$ either it contains a $G_{m}$ which is almost a $K_{m}$;

$\left(b_{2}\right)$ or a $G_{m}$ which is almost a $K(m / 2-\varepsilon m, m+\delta m)$.

(c) In both cases, if many edges connect $G_{n}-G_{m}$ to $G_{m}$, then we can embed $T_{k}$ into $G_{n}$, embedding a smaller part of $T_{k}$ outside of $G_{m}$, a larger part in the dense $G_{m}$, concluding that $T_{k} \hookrightarrow G_{n}$. 
(d) If, on the other hand, we have found such a "mini-almost-extremal" $G_{m} \subseteq G_{n}$, but $e\left(G_{m}, G_{n}-G_{n}\right)$ is "small", then we prove that

$$
e\left(G_{n}-G_{m}\right)>\frac{1}{2}(n-m)(k-2) .
$$

Hence we may forget the larger $G_{n}$ : replace it by the smaller $G_{n}-G_{m}$. (In other words, we can apply induction on $n$.)

(e) The real difficulty comes when we have sparse graphs: $e\left(G_{n}\right)=o\left(n^{2}\right)$. Then we partition $V\left(G_{n}\right)$ into three parts: $\mathbb{C}$ contains the vertices of high degrees, $\mathbb{B}$ contains a part of $V\left(G_{n}\right)$ not containing dense subgraphs, and therefore behaving in a pseudo-random way, and $\mathbb{A}$ behaves very similarly to the graphs we have in the dense cases.

\section{How do we handle the dense case?}

(i) Applying the Regularity Lemma to $G_{n}$, we get a so called Cluster Graph $H_{\nu}$. If this cluster graph has an (almost)-1-factor, then we can relatively easily embed $T_{k}$ into $G_{n}$, using the extra $\eta k n$ edges of (6.3).

(ii) Next we extend this case to a more general situation, when $G_{n}$ contains a so called Generalized 1-factor. We can prove the $\eta$-weakening in this case as well.

(iii) If the Cluster Graph $H_{\nu}$ does not contain an almost-1-factor, then we apply the Gallai-Edmonds structure-theorem (on graphs without 1-factors) to $H_{\nu}$. In this case we can either embed $T_{k}$ into $G_{n}$ directly, or reduce this case to Case (ii) above. Case (iii) is a very important subcase, with 3-4 subsubcases (depending on, how do we count them). Some of them go back to Case (ii) and in some others we directly (pseudo-greedily) embed $T_{k}$ into $G_{n}$.

\subsection{Komlós-Sós conjecture on median degree}

The Komlós-Sós conjecture was already formulated in Section 1.4. This is a generalization of the Loebl conjecture:

Conjecture 6.6 (Loebl-Komlós-Sós Conjecture [79]). If $G_{n}$ has at least $n / 2$ vertices of degree at least $k-1$, then $G_{n}$ contains all the $k$-vertex trees $T_{k}$.

The authors of [143] plan to write up the sharp version as well, which asserts the following.

Theorem 6.7. If $k$ is sufficiently large, then the Loebl-Komlós-Sós conjecture is true. 
Remarks 6.8. (a) The Loebl conjecture originates from a paper of Erdős, Füredi, Loebl, and T. Sós, on the discrepancy of trees [79].

(b) Pósa's theorem on the existence of Hamiltonian cycles also is - in some sense - a theorem asserting that if $G$ has many vertices of sufficiently high degree, then it is Hamiltonian. There were earlier cases, when Woodall 250, proved an Erdős-Gallai type theorem on cycles, using the condition that there are many vertices of high degree. Also, Erdös, Faudree, Schelp, and Simonovits - trying to prove some Ramsey type theorems, - found a similar statement [78], but not for all the trees, only for the paths, and they proved there an almost sharp theorem. Their sharp conjecture was later proved by Hao Li.

(c) There were many important steps to reach the theorem above. We should mention here Ajtai-Komlós-Szemerédi, 4], then Yi Zhao [255], next Piguet and Stein [201], 202], Cooley [54], Hladký and Piguet [144], and many others.

\section{More complex excluded subgraphs}

In this section we present three theorems, each leading to a reduction method to prove new results from old estimates. Still there is no general theory to determine the bipartite Turán numbers.

The three results we pick are the Erdős-Simonovits cube theorem (Theorem 7.1), $\operatorname{ex}\left(n, Q_{8}\right)=O\left(n^{8 / 5}\right)$ which led to the Erdős-Simonovits reduction, the Faudree-Simonovits theorem (Theorem 7.8 ) concerning Theta graphs, a generalization of the Erdős-Bondy-Simonovits theorem, $\mathbf{e x}\left(n, C_{2 k}\right)=O\left(n^{1+(1 / k)}\right)$, and Füredi's theorem (Theorem 7.15) on two levels of the Boolean lattice which implies a general upper bound $\mathbf{e x}(n, L)=O\left(n^{2-(1 / r)}\right)$ for any graph $L$ with vertices of degrees at most $r$ on one side of $L$.

\subsection{The Erdős-Simonovits Reduction and the Cube theorem}

We have already mentioned Theorem 1.5, on the extremal number of the cube. Here we formulate a sharpening of it.

Theorem 7.1 (90]). Let $Q_{8}$ denote the graph determined by the 8 vertices and 12 edges of a cube, and $Q_{8}^{+}$denote the graph obtained by joining two opposite vertices of this cube. Then

$$
\operatorname{ex}\left(n, Q_{8}\right) \leq \mathbf{e x}\left(n, Q_{8}^{+}\right)=O\left(n^{8 / 5}\right) .
$$




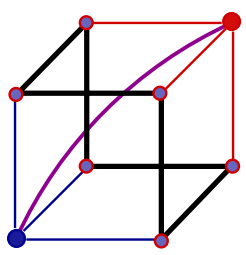

One reason why Erdös and Simonovits considered the extremal problem of the Cube graph was that this was one of Turán's originally posed problems. The reason that $Q_{8}^{+}$ was also considered was that Erdős and Simonovits got it for free: their proof of Theorem 1.5 gave the same upper bound for $Q_{8}^{+}$.

Let $L$ be a bipartite graph with partite sets $X$ and $Y$, and let $K_{t, t} * L$ denote the graph obtained by completely joining one partite set of $K_{t, t}$ to $X$ and the other to $Y$.

Theorem 7.2 (Erdős and Simonovits Reduction Theorem [90]). If $L$ is a bipartite graph with $\mathbf{e x}(n, L)=O\left(n^{2-a}\right), a \leq 1$, and $b$ is defined by $\frac{1}{b}=\frac{1}{a}+t$, then $\operatorname{ex}\left(n, K_{t, t} * L\right)=O\left(n^{2-b}\right)$.

The proof can go by induction on $t$ and by counting the number of $C_{4}$ 's.

Let $H$ be the graph obtained by deleting just three independent edges from $K_{4,4}$. Since $H=K_{1,1} * C_{6}$, Theorem 7.2 and $\operatorname{ex}\left(n, C_{6}\right)=O\left(n^{4 / 3}\right)$ (Corollary 4.9) imply Theorem 7.1 .

Since $\operatorname{ex}(n, L)=O(n)$ if $L$ is a tree, so we have the following result:

Corollary 7.3. For any tree $L, \operatorname{ex}\left(n, K_{t, t} * L\right)=O\left(n^{2-(1 /(t+1))}\right)$.

This can be considered as a generalization of

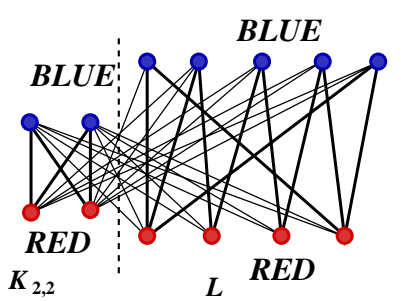
the Kövári-T. Sós-Turán theorem, since for $L=$ $K_{2}$ we have $K_{t+1, t+1}=K_{t, t} * K_{1,1}$. Since $Q_{8}-e$ is a subgraph of $K_{1,1} * P_{6}$, Corollary 7.3 implies

Corollary 7.4 (Erdős and Simonovits). $\operatorname{ex}\left(n, Q_{8}-e\right)=$ $O\left(n^{3 / 2}\right)$.

Further,

Theorem 7.5 (Erdős). Delete an edge from $K_{a, a}$. For the resulting $L=K_{a, a}-e$ we have $\mathbf{e x}(n, L)=O\left(n^{2-\frac{1}{a-1}}\right)$.

Indeed, for $a \geq 3$ the graph $K_{a, a}-e$ is a subgraph of $K_{a-2, a-2} * P_{4}$.

Since $K_{b, b}-K_{a, a}($ for $b-2 \geq a \geq 1)$ can be written as $K_{b-a-1, b-a-1} * T$ where $T$ is a double star, Corollary 7.3 also implies that $\mathbf{e x}\left(n, K_{b, b}-K_{a, a}\right)=$ $O\left(n^{2-(1 /(b-a))}\right)$. For this important case Füredi and West gave a sharper upper bound.

Theorem 7.6 ([121]). For every $n \geq b>a$ we have

$$
\operatorname{ex}\left(n, K_{b, b}-K_{a, a}\right) \leq \frac{1}{2}(b+a-1)^{1 /(b-a)} n^{2-(1 /(b-a))}+\frac{1}{2}(b-a-1) n .
$$


In particular, it gives $\mathbf{e x}\left(n, K_{3,3}-e\right) \leq \frac{1}{2} \sqrt{3} n^{3 / 2}+O(n)$. This was further improved by J. Shen [214] to

$$
\operatorname{ex}\left(n, K_{3,3}-e\right) \leq \frac{\sqrt{15}}{5} n^{3 / 2}+O(n) .
$$

He also showed that $\operatorname{ex}\left(n, n, K_{3,3}-e\right) \leq(4 / \sqrt{7}) n^{3 / 2}+(n / 2)$.

Pinchasi and Sharir extended the cube theorem, using a somewhat different proof:

Theorem 7.7 (Pinchasi and Sharir [204]). A bipartite graph $G[A, B]$ with $|A|=m$ and $|B|=n$, not containing the cube $Q$ has

$$
O\left(n^{4 / 5} m^{4 / 5}+m n^{1 / 2}+n m^{1 / 2}\right)
$$

edges.

Another, more explicit proof for Theorem 7.7 was presented in [115].

\section{Historical remarks:}

(a) Erdős and Simonovits first proved the Cube theorem, using the $\Delta$ almost-regularization.

(b) It seems that Theorem 7.2 covered all the cases known until that point.

(c) This (i.e. the Cube Recursion Theorem) was the first case, where one got an exponent, different from $2-(1 / a)$ and $1+(1 / a)$. Actually, Erdös thought earlier, that all the exponents must be of this form, (see [70]). This was disproved in their paper [90]: not by the cube, since there is no good lower bound for the cube: even $\operatorname{ex}\left(n, Q_{8}\right) / n^{3 / 2} \rightarrow \infty$ is not known. However, a more complicated example, for which the lower bound - using random graphs - was good enough, disproved Erdős' conjecture. Actually, one thinks that each rational $\alpha \in(0,1)$ is extremal exponent for some finite $\mathcal{L}_{\alpha}$, see Conjecture 2.37 .

To disprove the Erdös conjecture concerning the exponents are of the form $1+(1 / a)$ or $2-(1 / a)$ it is enough to notice that we have graphs $H$ with

$$
c_{H} n^{(8 / 5)-\varepsilon(H)}<\operatorname{ex}(n, H)=O\left(n^{8 / 5}\right), \quad\left(c_{H}>0, \varepsilon(H)<\frac{1}{10}\right) .
$$




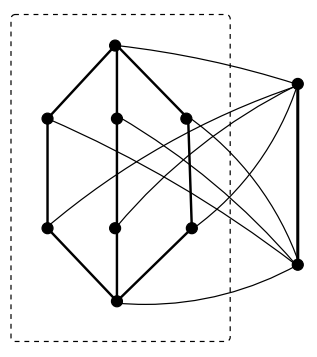

More generally, consider the graph $H(t, \ell)$ obtained by connecting a $\Theta(3, \ell)$ to $K(t, t)$, as described in Theorem 7.2. By the Theorem 2.26 (lower bound) and Theorem 7.2 and Theorem 7.8 (upper bound) we obtain

$$
c_{\ell, t} n^{2-\frac{2 \ell+2 t}{3 \ell+t^{2}+2 t(\ell+1)-1}}<\operatorname{ex}\left(n, H_{t, \ell}\right) \leq \tilde{c}_{\ell, t} n^{2-\frac{2}{2 t+3}} .
$$

So all the numbers $2-\frac{2}{2 t+3}$ are points of accumulations of exponents, in this sense. Actually, applying this argument with $t=1, \ell=3$, we get a simple counterexample, with the upper bound $O\left(n^{8 / 5}\right)$ and a lower bound $c n^{2-(8 / 17)}(c>0)$.

(d) In [92, Erdős and Simonovits proved the Supersaturated graph theorem (see Section 11) corresponding to the cube, thus providing a second proof of the Cube Theorem, that needed "less regularization".

\subsection{Theta graphs and the Faudree-Simonovits reduction}

There is an alternative proof for the Bondy-Simonovits Theorem in [99]. This proof enabled a generalization to $\Theta$-graphs. Recall that $\Theta_{k, \ell}$ denotes the graph consisting of $\ell$ paths of length $k$ with the same endpoints but no inner intersections. We have $v\left(\Theta_{k, \ell}\right)=2+(k-1) \ell$ and $e\left(\Theta_{k, \ell}\right)=k \ell$.

Theorem 7.8 (Theta-graph, Faudree-Simonovits [99]). For fixed $k$ and $\ell \geq 2$ one has $\operatorname{ex}\left(n, \Theta_{k, \ell}\right)=O\left(n^{1+(1 / k)}\right)$.

This exponent is conjectured to be the best possible,

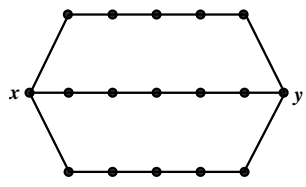
see Conjecture 4.11.

Applying the Erdős-Rényi Random Lower bound (Theorem 2.26) in its simpler form to $\Theta_{k, \ell}$ we get

$$
\operatorname{ex}\left(n, \Theta_{k, \ell}\right)>c_{k, \ell} n^{1+\frac{1}{k}-\frac{2}{k \ell}}
$$

asymptotically matching the upper bound's exponent.

The proof of Theorem 7.8 came from a "Recursion" theorem, asserting that if one knows good upper bounds for an $L$, and $L^{*}$ is built from $L$ in a simple way, then one has a good upper bound on $\operatorname{ex}\left(n, L^{*}\right)$ as well.

Definition 7.9. Let $L$ be a bipartite graph, with a fixed 2-colouring $\psi$ in RED-BLUE with $h$ RED vertices. Let $x \notin V(L)$ be a vertex from which $h$ independent paths of $k-1$ edges go the RED vertices of $L$, (these paths intersect only in $x)$. Denote the obtained graph by $L_{k}(L, \psi)$. 
Theorem 7.10 (Faudree-Simonovits Reduction, Trees [99]). If $L$ is a tree, then

$$
\operatorname{ex}\left(n, L_{k}(L, \psi)\right)=O\left(n^{1+(1 / k)}\right)
$$

The Theta graph $\Theta_{k, \ell}$ is obtained from a star of $\ell$

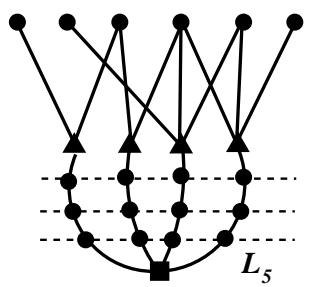
edges. One has to be cautious with the next theorem, see Remark 7.12 ,

Theorem 7.11 (Faudree-Simonovits Reduction, General Case [99, 100]). Let $L$ be an arbitrary bipartite graph with a fixed coloring $\psi$ and assume that

$$
\operatorname{ex}^{*}(n, L)=O\left(n^{2-\alpha}\right) \text {. }
$$

Then for

$$
\beta=\frac{\alpha+\alpha^{2}+\cdots+\alpha^{k-2}}{1+\alpha+\alpha^{2}+\cdots+\alpha^{k-2}}
$$

we have

$$
\operatorname{ex}\left(n, L_{k}(L, \psi)\right) \leq \mathbf{e x}^{*}\left(n, L_{k}(L, \psi)\right)=O\left(n^{2-\beta}\right) .
$$

Remark 7.12. Most probably, this recursion is never sharp but for trees. In its proof one has to apply standard arguments to subgraphs of $K(m, n)$ where $n \gg m$. We very seldom have matching lower and upper bounds in such cases.

\subsection{A universal graph and dependent random choice}

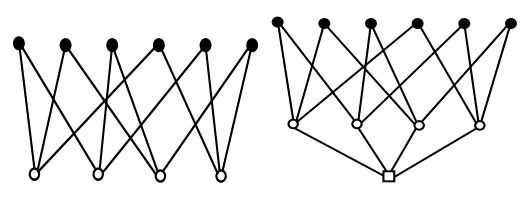

Erdős asked the following question: what are the extremal numbers for the two graphs on the left: The left one will be called $M_{10}$, the right one $M_{11}$ and they are described as special cases of the following

Definition 7.13. Let $k, r$ and $t$ be given positive integers. $U(k, r, t)$ is obtained from the $k$ vertices $x_{1}, \ldots, x_{k}$ by joining to each of the $r$-element subsets of $\left\{x_{1}, \ldots, x_{k}\right\} t$ distinct vertices $y_{i_{1}, \ldots, i_{r}}^{i} . U^{+}(k, r, t)$ is obtained from $U(k, r, t)$ by joining a new vertex $w$ to all $x_{h}, h=1, \ldots, k$.

Problem 7.14 (Erdős). Determine (or estimate) $\operatorname{ex}(n, L)$ for $L:=M_{10}=$ $U(4,2,1)$ and $L:=M_{11}=U^{+}(4,2,1)$. 
One could ask for the motivation: why these graphs? Perhaps having obtained the cube theorem, we had good upper and lower bounds only in very special cases, when $L$ contained some sample graphs - say a $C_{4}$ for which we have already provided sharp lower bounds. $U(4,2,1)$ clearly needed a new approach, and e.g. $U^{+}(k, 2,1)$ contains many $C_{4}$ 's but the earlier methods did not yield appropriate upper bounds. Füredi [107] answered this question proving that $\left.\operatorname{ex}\left(n, U^{+}(k, 2,1)\right)\right)<k^{3 / 2} n^{3 / 2}$. More generally,

Theorem 7.15 (Füredi [107]). Let $U^{+}(k, r, t)$ be the universal bipartite graph from Definition 7.13. Then there exists $a c=c_{r}^{k, t}>0$ such that

$$
\operatorname{ex}\left(n, U^{+}(k, r, t)\right)<c n^{2-(1 / r)} .
$$

Concerning the Erdös question we have $\operatorname{ex}\left(n, U^{+}(k, 2,1)\right)<k^{3 / 2} n^{3 / 2}$ and more generally

$$
\operatorname{ex}\left(n, U^{+}(k, 2, t)\right)<n^{3 / 2} \cdot \sqrt{\frac{t k(k-1)^{2}+2(k-2)(k-1)}{8}}+n \frac{k-1}{4} .
$$

Multiplying each vertex $(k-1)$ times in a $C_{4}$-free graph we get a $U^{+}(k, 2,1)$ free graph which yields $\mathbf{e x}\left(n, U^{+}(k, 2,1)\right) \geq \Omega\left(k^{1 / 2} n^{3 / 2}\right)$.

Erdös had the more general conjecture

Conjecture 7.16 (Erdös, 66], see also [92], 225]). If every subgraph of the bipartite graph $L$ has a vertex of degree at most $r$, then

$$
\operatorname{ex}(n, L)=O\left(n^{2-(1 / r)}\right) .
$$

The upper bound (7.3) for the universal graph immediately gives

Corollary 7.17. If $L$ is bipartite and has a 2-coloring where in the first color class all but one vertex is of degree at most $r$, then

$$
\operatorname{ex}(n, L)=O\left(n^{2-(1 / r)}\right) .
$$

Indeed, all such graphs can trivially be embedded into an appropriate $U^{+}(k, r, t)$.

Alon, Krivelevich, and Sudakov [9] gave a new probabilistic proof (for graphs where on one side all vertices are of degree at most $r$ ) with a better constant $c_{r}^{k, t}$. Their proof method became known as "dependent random choice"; for a survey see [101]. 
Lemma 7.18 (Dependent random choice, see, e.g., [101]). Let $k, t, r$ be positive integers. Let $G_{n}$ be a graph with $n$ vertices and average degree $d, d$ be an integer. If there is a positive integer a such that

$$
\frac{d^{a}}{n^{a-1}}-\left(\begin{array}{l}
n \\
r
\end{array}\right)\left(\frac{t}{n}\right)^{a} \geq k
$$

then $G_{n}$ contains a subset $U$ of at least $k$ vertices such that every $r$ vertices in $U$ have at least $t$ common neighbors.

Note that in this lemma they do not claim that $U(k, r, t)$ is a subgraph. Nevertheless, using this lemma they improve the constant $c=c_{r}^{k, t}$ in (7.3) from $O\left((t+1)^{1 / r} k^{2-(2 / r)}\right)$ to $c \leq 2^{-1+(2 / t)}(t+1)^{1 / r} k$.

As they mention at the end of [9], both proofs of Theorem 7.15 give a bit more (and thus Corollary 7.17 can be sharpened accordingly):

$$
\operatorname{ex}\left(n, U^{+r}(k, r, t)\right)<c n^{2-(1 / r)},
$$

where the graph $U^{+r}$ is obtained from $U^{+}(k, r, t)$ by replacing the vertex $w$ in Definition 7.13 by an independent set of $r$ vertices with the same neighbors, $x_{1} \ldots, x_{k}$.

However, the method of Dependent random choice gives more. Call a graph $L_{h}$ on $h$ vertices $r$-degenerate if it satisfies the condition of Conjecture 7.16. In other words, there is an ordering of its vertices $x_{1}, \ldots, x_{h}$ such that for every $1 \leq i \leq h$ the vertex $x_{i}$ has at most $r$ neighbors $x_{j}$ with $j<i$.

Theorem 7.19 (Alon, Krivelevich, and Sudakov [9]). If $L$ is bipartite $r$ degenerate graph on $h$ vertices, then for every $n \geq h$

$$
\operatorname{ex}(n, L) \leq h^{1 /(2 r)} n^{2-(1 / 4 r)} .
$$

Applying the above results with $r=2, t=1$ and $k=c \sqrt{n}$ to find a $U(k, 2,1)$ one immediately obtains the following. Any graph on $n$ vertices with $c_{1} n^{2}$ edges contains a 1-subdivision of $K_{k}$ with $k=c_{2} \sqrt{n}$ for some positive $c_{2}$ depending on $c_{1}$. This answers a question of Erdős [72]. The theorems of Bollobás and Thomason [29] and Komlós and Szemerédi [161] also imply the existence of such a large topological clique but their subgraph is not necessarily a 1-subdivision.

Given any graph $L$, let $\bar{d}$ denote the $\max _{X \subseteq V(L)}\left\{2 e_{L}(X) /|X|\right\}$, the maximum local average degree. Then $L$ is $\lfloor\bar{d}\rfloor$-degenerate. Hence the upper bound of Theorem 7.19 and the random method lower bound in (2.9) yield that 
Corollary 7.20. For every bipartite graph $L$,

$$
\Omega\left(n^{2-c}\right) \leq \mathbf{e x}(n, L) \leq O\left(n^{2-(c / 8)}\right),
$$

where $c=2 / \bar{d}$, is the same as in (2.9).

\section{Eigenvalues and extremal problems}

Let $A=A\left(G_{n}\right)$ be the adjacency matrix of $G_{n}$, and $\mathbf{j}$ be the vector each entry of which is 1 . Since

$$
e\left(G_{n}\right)=\frac{1}{2} \mathbf{j} A \mathbf{j}^{T}
$$

and, more generally,

$$
w_{k}\left(G_{n}\right)=\frac{1}{2} \mathbf{j} A^{k} \mathbf{j}^{T}
$$

counts the number of $k$-edge walks in $G_{n}$, therefore it is not so surprising that eigenvalues can be used in extremal graph problems. An easy to read source on spectra of graphs is Cvetkovič-Doob-Sachs [56].

Theorem 8.1 (Babai-Guiduli [12]). Let $\Lambda(G)=\max \left|\lambda_{i}\right|$, where $\lambda_{1}, \ldots, \lambda_{n}$ are the eigenvalues of $A\left(G_{n}\right)$. If $K_{a, b} \nsubseteq G_{n}$, (and $2 \leq a \leq b$ ) then

$$
\Lambda \leq \sqrt[a]{b-1} \cdot n^{1-(1 / a)}+o\left(n^{1-(1 / a)}\right) .
$$

Since trivially

$$
2 e\left(G_{n}\right) \leq \Lambda n
$$

the inequality (8.3) implies Theorem 2.22 apart from the $o()$ term.

Remark 8.2. For regular or almost regular graphs $\Lambda\left(G_{n}\right) \approx \frac{2 e\left(G_{n}\right)}{n}$, and then the two estimates are basically equivalent. The constant in the above theorem is not sharp since - as we know from Theorem 3.19, - the constant can be improved.

We have already mentioned Nikiforov's result (Theorem 3.21) on the Zarankiewicz problem. In fact, he proved [198] that for all $n \geq b \geq a \geq 2$ and a $K_{a, b}$-free graph $G_{n}$ we have

$$
\Lambda\left(G_{n}\right) \leq(b-a+1)^{1 / a} n^{1-(1 / a)}+(a-1) n^{1-(2 / a)}+(a-2) .
$$

This improves the coefficient in Theorem 8.1. It also implies Füredi's bound (3.11) for the $\operatorname{ex}\left(n, K_{a, b}\right)$ according to (8.4). For $C_{4}$-free graphs he has $\Lambda^{2}-\Lambda+1 \leq n$. 
Recall that $T_{n, k}$ denotes the Turán graph, the $k$-partite complete graph of maximum size. Given a $K_{k+1}$-free graph $G_{n}$ Nikiforov [197] showed that $\Lambda\left(G_{n}\right)<\lambda\left(T_{n, k}\right)$ unless $G=T_{n, k}$. For a recent reference of a generalization see Z. L. Nagy [196].

\section{Excluding topological subdivisions}

\subsection{Large topological subgraphs}

We have already mentioned that our classification does not hold for infinite families of excluded subgraphs. One important phenomenon is that $\operatorname{ex}(n, \mathcal{L})$ can be linear for infinite $\mathcal{L}$ even if $\mathcal{L}$ contains only cycles. 28 Here we consider a very central graph theoretical problem strongly connected to the 4-colour conjecture.

Definition 9.1. Given a graph $H$, its subdivision (or a topological $H$ ) is obtained from it by replacing each edge $e$ of $H$ by some paths $P_{e}$ so that these paths do not have their inner (new) vertices in common.

Wagner asked if for any integer $\ell$ there exists a $k=k_{\ell}$ such that any $G$ with chromatic number $\chi(G)>k_{\ell}$ must contain a topological subdivision of $K_{\ell}$. This was proved by Gabor Dirac and H. Jung (independently). Answering a question of Dirac, Mader proved the following important result.

Theorem 9.2 (Mader, [183]). If $G_{n}$ is an n-vertex graph, and

$$
e\left(G_{n}\right) \geq n(\ell-1) 2^{\left(\begin{array}{l}
\ell-1 \\
2
\end{array}\right)-1},
$$

then $G_{n}$ contains a subdivision of the complete $\ell$-graph.

This statement is stronger than the original Wagner conjecture, since a graph with large chromatic number contains a subgraph with large minimum degree. Mader, and independently, Erdős and Hajnal conjectured that

Conjecture 9.3 (Mader, Erdös-Hajnal). There exists a constant $c>0$ such that if $e\left(G_{n}\right)>c \ell^{2} n$, then $G_{n}$ contains a topological $K_{\ell}$.

A slightly weaker form of this conjecture was proved by Komlós and Szemerédi, [160], then - by a different method - Bollobás and Thomason 29. proved this conjecture and, almost immediately after that, Komlós and Szemerédi [161] proved Conjecture 9.3 as well.

\footnotetext{
${ }^{28}$ Here the simplest case is Theorems 5.1
} 
Theorem 9.4 (Bollobás-Thomason). Every graph $G_{n}$ of size at least $256 \ell^{2} n$ edges contains a topological complete subgraph of order $\ell$.

As to the small values of $\ell$, Dirac conjectured that for $n \geq 3$ every $G_{n}$ with $e\left(G_{n}\right) \geq 3 n-5$ contains a topological $K_{5}$. This improvement of the famous Kuratowski theorem was proved by Mader in [184] and the corresponding extremal graphs were characterized in [186]. The reader is recommended the excellent "featured review" of Carsten Thomassen on the paper of Mader [184, on the MathSciNet.

An excellent survey of Mader on this topic is [185].

\subsection{Turán numbers of subdivided graphs}

Let $\varepsilon$ be a positive real, $0<\varepsilon<1$. Kostochka and Pyber 163 proved that every $n$-vertex graph $G_{n}$ with at least $4^{t^{2}} n^{1+\varepsilon}$ edges contains a subdivision of $K_{t}$ on at most $\left(7 t^{2} \ln t\right) / \varepsilon$ vertices, where $0<\varepsilon<1$. This (for $t=5$ ) answers a question of Erdős about finding a non-planar subgraph of size $c(\varepsilon)$ in a graph with $n^{1+\varepsilon}$ edges.

Recently, T. Jiang [150] improved the Kostochka-Pyber upper bound to $O\left(t^{2} / \varepsilon\right)$. On the other hand, for each $0<\varepsilon<1$ and $n>n_{0}(\varepsilon)$ there are $n$ vertex graphs of girth at least $1 / \varepsilon$ (see Corollary 2.30). In such a graph any subdivision of $K_{t}$ must contain $\Omega\left(t^{2} / \varepsilon\right)$ vertices, so Jiang's result is sharp.

Theorem 9.5 (Jiang and Seiver [151]). Let $L$ be a subdivision of another graph $H$. For each edge $x y \in E(H)$ let $\ell(x, y)$ denote the length of the path in $L$ replacing the edge $x y$. Suppose that $\ell(x, y)$ is even for each edge of $H$, and let $\min \{\ell(x, y): x y \in E(H)\}=2 m$. Then $\operatorname{ex}(n, L)=O\left(n^{1+(8 / m)}\right)$.

The main tools in the proof are the Dependent Random Choice, Lemma 7.18, and the Erdős-Simonovits $\Delta$-almost-regularization, Theorem 2.19 .

\section{Hypergraph Extremal Problems}

\subsection{Positive Density problems}

This is a short detour into Hypergraph Extremal Problems. Now our "Universe" is the class of $r$-uniform hypergraphs. Katona, Nemetz and Simonovits [154] showed (using a simple averaging) that

Theorem 10.1 (Katona, Nemetz and Simonovits [154]). $\operatorname{ex}_{r}(n, \mathcal{L}) /\left(\begin{array}{l}n \\ r\end{array}\right)$ is monotone decreasing, and therefore convergent. 


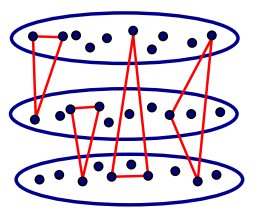

The hypergraph extremal problems are extremely hard. Even the simplest extension of Turán's theorem is unsolved: Let $K_{4}^{(3)}$ be the three-uniform hypergraph with 4 vertices and 4 triples.

Construction 10.2 (Turán, the simplest case). We partition $n$ vertices into three classes $C_{1}, C_{2}, C_{3}$ and we take all the triples of the form $(x, y, z)$, where

(a) $x \in C_{i}, y \in C_{i}, z \in C_{i+1}$ (where the indices are taken mod 3);

(b) the three vertices are in three different groups.

One can easily see that his construction contains no $K_{4}^{(3)}$.

Conjecture 10.3 (Turán). Construction 10.2 is asymptotically extremal for $K_{4}^{(3)}$. (Perhaps it is extremal, not only asymptotically extremal, at least for $n>n_{0}$.)

Here we cut it short and recommend the reader (among others) the survey of Füredi on hypergraph extremal problems [108], and also the papers of Füredi and Simonovits [120, Keevash and Sudakov [156], Füredi-PikhurkoSimonovits [119], and the survey of Keevash [155].

\subsection{Degenerate hypergraph problems}

For $r$-uniform hypergraphs the $r$-partite graphs generalize the bipartite graphs. An important illustration of this is the one below, extending Theorem 2.31 .

Theorem 10.4 (Degenerated hypergraph problems). For an $r$-uniform extremal hypergraph problem of $\mathcal{L}^{(r)}, \operatorname{ex}\left(n, \mathcal{L}^{(r)}\right)=o\left(n^{r}\right)$, if and only if there is an $L \in \mathcal{L}^{(r)}$ which can be $r$-vertex-colored so that each hyperedge of $L$ gets $r$ distinct colors.

Theorem 10.4 is an easy corollary of the following theorem of Erdös, (which generalizes Theorem 2.22).

Theorem 10.5 (Erdös [65]). Let $K^{(r)}\left(a_{1}, \ldots, a_{r}\right)$ be the r-uniform hypergraph with $r$ vertex-classes $C_{1}, \ldots, C_{r}$, where $\left|C_{i}\right|=a_{i}$, and $a_{1}=t$. Then

$$
\mathbf{e x}^{(r)}\left(n, K^{(r)}\left(a_{1}, \ldots, a_{r}\right)\right)=O\left(n^{r-\left(1 / t^{r-1}\right)}\right) \text {. }
$$

Extending some problems and results for ordinary graphs, Brown, Erdős and Sós started investigating the following 
Problem 10.6 (Brown, Erdős, and Sós [39], 40]). Consider $r$-uniform hypergraphs for some fixed $r$, and denote by $\mathbb{H}_{k, \ell}^{r}$ the family of $r$-uniform $k$ vertex hypergraphs with $\ell$ hyperedges. Determine or estimate $f_{r}(n, k, \ell):=$ $\operatorname{ex}\left(n, \mathbb{H}_{k, \ell}^{r}\right)$.

Brown, Erdős, and Sós proved many upper and lower bounds for special cases of Problem 10.6. We have already mentioned one of them: the $f_{3}(n, 6,3)$-problem 29 It is easy to see that $f_{3}(n, 6,3)<\frac{1}{6} n^{2}$. The real question was if $f_{3}(n, 6,3)=o\left(n^{2}\right)$ or not. Ruzsa and Szemerédi [210] proved that the answer is YES. We formulated this in Theorem 1.9, This theorem became a very important one. We originate, among others, the "Removal Lemma" from here.

We shall return to this problem in the section on applications.

\section{Supersaturated graphs}

The theory of Supersaturated extremal problems is a very popular area today. Here we shall restrict ourselves to the supersaturated extremal graph problems related to bipartite excluded graphs, just mention a few further references, like Lovász and Simonovits [178], Razborov [179], Lovász [177], Reiher [205].

Given a graph $G$, denote by $N(G, F)$ the number of subgraphs of $G$ isomorphic to $F$. Here we have to be slightly cautious: if $F$ has non-trivial automorphisms, then we can count isomorphisms or isomorphic subgraphs, and the ratio of these two numbers equal to the automorphism number.

A theorem which asserts that a graph $G_{n}$ contains very many graphs $L$ from a family $\mathcal{L}$ is called a theorem on supersaturated graphs. Such theorems are not only interesting in themselves, but also are often useful in establishing other extremal results. At this point it is worthwhile mentioning such a result for complete bipartite graphs, obtained by Erdös and Simonovits [94]:

Theorem 11.1 (Number of complete bipartite graphs). For any integers a and $b$ there exists a constant $c_{a, b}>0$ such that if $G_{n}$ is a graph with e edges, then $G_{n}$ contains at least $\left[c_{a, b} e^{a b} / n^{2 a b-a-b}\right]$ copies of $K_{a, b}$.

Corollary 11.2. Let $c>0$. If $e\left(G_{n}\right)=e>(1+c) \operatorname{ex}\left(n, C_{4}\right)$, then $G_{n}$ contains at least $\gamma e^{4} / n^{4}$ copies of $C_{4}$, for some $\gamma(c)>0$. The random graph with e edges shows that this is sharp.

\footnotetext{
${ }^{29}$ If $r=3$, then we delete the subscript in $f_{3}$.
} 
Proof of the Cube Theorem (Sketch). Apply Theorem 2.19 obtaining a $\Delta$-almost-regular (bipartite) $\tilde{G_{n}} \subseteq G_{n}$. Apply the corollary to this $\tilde{G}_{n}$. It contains $\gamma \frac{e^{4}}{n^{4}} C_{4}$ 's. On the average, an edge of $G_{n}$ is contained in $\gamma e^{3} / n^{4}$ copies of $C_{4}$. Take a typical edge $x y$ : the bipartite graph $G[U, V]$ spanned by the neighbors $U:=N(x)$ and $V:=N(y)-$ by $\operatorname{ex}\left(m, C_{6}\right)=O\left(m^{4 / 3}\right),-$ will contain a $C_{6}$. Now, $x y$ and this $C_{6}$ will provide a $Q_{8}^{+}$: a cube with a diagonal.

Basically the same argument proves Theorem 7.2 ,

\subsection{Erdős-Simonovits-Sidorenko conjecture}

In this part $\chi(L)=2$. Erdös and Simonovits [225] formulated three conjectures and also that the main idea behind these conjectures is that the number of copies of subgraphs $L \in G_{n}$ is minimized by the random graph if $E=e\left(G_{n}\right)$ is fixed and is not too small.

To formulate these conjectures, first we calculate the "expected number of copies" of $L \subseteq R_{n}$ if $R_{n}$ is a random graph with edge probability $p=$ $E /\left(\begin{array}{l}n \\ 2\end{array}\right)$. Let $v=v(L)$, and $e=e(L)$. Clearly, if the edges are selected independently, with probability $p$, then $K_{n}$ contains $\left(\begin{array}{l}n \\ v\end{array}\right)$ possible $v$-tuples, each containing the same number $a_{L}$ of copies of $L$, and therefore

$$
\mathbb{E}\left(\#\left(L \subseteq R_{n}\right)\right)=\left(a_{L}+o(1)\right) \frac{n^{v}}{v !} p^{e}=\left(a_{L}+o(1)\right) \frac{n^{v}}{v !}\left(\frac{2 E}{n^{2}}\right)^{e}=a_{L}^{*} \frac{E^{e}}{n^{2 e-v}}
$$

Conjecture 11.3 (Erdős-Simonovits, 225]). There are two constants, $\Omega=$ $\Omega_{L}>0$ and $c=c_{L}>0$ such that if $E>\Omega \cdot \operatorname{ex}(n, L)$, then any graph $G_{n}$ with $E$ edges contains at least

$$
c_{L} \frac{E^{e}}{n^{2 e-v}}
$$

copies of $L$.

This was the weakest form. The strongest form of this conjecture was

Conjecture 11.4 (Erdős and Simonovits). For every $\varepsilon>0$, if $E>(1+\varepsilon)$. $\operatorname{ex}(n, L)$, then any graph $G_{n}$ with $E$ edges contains at least $(1+\varepsilon) \mathbb{E} \mathbb{R}(n, L, E)$ copies of $L$, if $n>n_{0}(\varepsilon)$, where $\mathbb{E} \mathbb{R}(n, L, E)$ denotes the expected number of edges of a random Erdös-Rényi graph with $n$ vertices and $E$ edges.

Obviously, one has to assume that $e\left(G_{n}\right)>\operatorname{ex}(n, L)$. 
Remark 11.5 (Relation to Sidorenko's Conjecture). At first sight Sidorenko's conjecture [216] seems to be sharper than the above one. This is not the case. In fact, Sidorenko's Conjecture applies only to dense host graphs. There, as Sidorenko points out in his papers, the two versions are equivalent.

Also, it is obvious that there is not much difference if we consider above the hypergeometric model of random graphs, where the number of vertices and edges are given, or if we fix only $n$ but the edges are taken independently, and therefore $e\left(G_{n}\right)$ follows a binomial distribution.

Sidorenko, working on applications of extremal graph theorem to probability distribution translated the above conjecture to integrals and arrived at a conjecture [216], where the error terms disappeared. The meaning of his version was that if one considers dense graphs and defines $L \subseteq R$ for the case when $G$ is a function, generalizing the notion of graphs, then the Random Continuous graph will have the least number of copies of $L$, more precisely, that will minimize the corresponding integral.

We skip the formulation of this problem, just refer to some papers of Lovász, and Hatami [140], and to the book of Lovász [177].

Jagger, Št́ovíček, and Thomason [148] investigated the following problem originating from a conjecture of Erdős, disproved by Thomason.

Problem 11.6. Given a sample graph $L$, denote by $\rho_{L}\left(G_{n}\right)$ the sum of copies of $L$ in $G_{n}$ and in its complementary graph. What is the minimum $\Gamma_{n}(L)$ of this, taken over all $n$-vertex graphs?

Erdös conjectured that the random graph yields the minimum, for $K_{4}$. This was disproved by Thomason [237. Investigating the case of general $L$, Jagger, Štovíček, and Thomason proved some interesting results in connection with Sidorenko's conjecture.

Here we should emphasize that there is a slight difference between looking for copies of an $L$ in $G_{n}$ or for copies of homomorphic images: In the second case we allow vertices to map into $G_{n}$ with some coincidences.

As to the Sidorenko Conjecture, the first unknown case (as Sidorenko mentions) is when we delete the edges of a Hamiltonian cycle from $K_{5,5}$.

Theorem 11.7 (Conlon, Fox, Sudakov [52] [53]). The Sidorenko Conjecture holds if $L=L[A, B]$ is a bipartite graph with a vertex $x \in A$ completely joined to $B$.

Remark 11.8. Unfortunately, we do not have sufficiently good lower bounds for the extremal problem of the cube. The Erdös-Simonovits Conjecture was proved for $Q_{8}$ in [92]. 
Hatami proved the Sidorenko conjecture for any cube (i.e. of any dimension), yet, that was not really enough to provide a reasonable upper bound for the 4-dimensional cube. This reflects some difference between extremal problems and the corresponding Supersaturated Graph Problems (at least, for dense host graphs).

\section{Ordered structures}

\subsection{Directed graphs, ordered graphs}

There is an extensive literature on Digraph extremal problems, see e.g., the survey of Brown and Simonovits [42], or [41]. We skip here the general theory.

Denote $\overrightarrow{\operatorname{ext}}(n, \vec{L})$ the maximum number of edges in a directed graph not containing the oriented subgraph $\vec{L}$. For every $\vec{L}$ containing a directed path of length 2 one has $\overrightarrow{\operatorname{ext}}(\vec{L}) \geq\left\lfloor n^{2} / 4\right\rfloor$. Indeed, orient the edges of $K_{n / 2, n / 2}$ simultaneously into one direction. For bipartite $\vec{L}$ it is more interesting to consider the minimum outdegree.

Consider the following directed graph $\vec{L}_{1, a, b}$ on $1+a+b$ vertices $w$, $x_{1}, \ldots x_{a}, y_{1}, \ldots, y_{b}$. The oriented edges are $w$ to $x_{i}$ and $x_{i}$ to $y_{j}(1 \leq i \leq a$, $1 \leq j \leq b)$.

Theorem 12.1 (Erdös, Harcos and Pach [82]). Given integers $a$ and $b$, there exists a $c=c_{a, b}>0$ such that the following holds. Any oriented graph with minimum out-degree $\delta^{+} \geq c n^{1-(1 / a)}$ contains a copy of $\vec{L}_{1, a, b}$.

This result opened up a new interesting field with many open problems.

Another ordered Turán function was defined by Timmons [239]. He showed that if a graph with vertex set $\{1,2, \ldots, n\}$ has at least

$$
(1+o(1))(2 / 3) n^{3 / 2}
$$

edges, then it contains a $C_{4}$ with vertices $a_{1} b_{1} a_{2} b_{2}$ such that $a_{1}, a_{2}<b_{1}, b_{2}$. He extended other ordinary Turán problems to these zig-zag type questions. Many problems remain unsolved.

\subsection{Erdős-Moser conjecture on unit distances}

Erdős and Leo Moser [83] conjectured that

Conjecture 12.2. If $n$ points of the plane are in convex position, then the number of unit distances among them is $O(n)$. 
Füredi proved a slightly weaker result:

Theorem 12.3 (Füredi [106]). If $n$ points are in convex position in the plane, then there are at most $O(n \log n)$ unit distances among them.

To prove this, Füredi directly formulated the excluded Ordered Matrix Property and solved a matrix-containment problem. The crucial point of his proof was Theorem 12.6 below.

The best known lower bound in the Erdős-Moser problem, $2 n-7$, is due to Edelsbrunner and P. Hajnal [58].

\section{3. $\quad$ Ordered submatrices}

The ordered matrix problems partly came from geometric problems (see Bienstock and Győri, [23, Füredi [106]), but they are interesting on their own, too. A geometric application, called Erdős-Moser conjecture, is discussed above in Subsection 12.2 .

We have already indicated that most extremal graph problems have matrix forms, too: To determine $\mathbf{e x}^{*}(m, n, L)$ we considered all $m \times n$ 0-1 matrices not containing any permutation of the bipartite adjacency matrix of $L$.

In the ordered case here we exclud only those submatrices where the indexing of the rows and columns of $\mathbf{M}$ is fixed. This way we exclude fewer subconfigurations.

Definition 12.4 (Matrix containment). Let $\mathbf{M}$ and $\mathbf{P}$ be two 0-1 matrices. We say that $\mathbf{M}$ contains $\mathbf{P}$ if we can delete some rows and columns of $\mathbf{M}$ and then perhaps switch some 1's into 0 so that the resulting matrix be $\mathbf{P}$. Otherwise we say that $\mathbf{M}$ avoids $\mathbf{P}$.

So, we can delete rows and columns of $\mathbf{M}$ but can not permute them. Now we can define the Matrix Extremal Problems:

Problem 12.5 (Ordered Matrix Problem). Given an $a \times b$ 0-1 (sample) matrix $\mathbf{P}$, and a (huge) $m \times n$ 0-1 matrix $\mathbf{M}$, how many 1's can occur in

$\mathbf{M}$ under the condition that $\mathbf{M}$ does not contain $\mathbf{P}$ in the "ordered" way. Denote by $\mathbf{e x t}_{\text {mat }}(m, n, \mathbf{P})$ the maximum.

One of the first nontrivial results was

Theorem 12.6 (Füredi [106]). Let

$$
\mathbf{P}=\left(\begin{array}{lll}
1 & 1 & 0 \\
1 & 0 & 1
\end{array}\right) .
$$


If the $n \times n$ 0-1 matrix $\mathbf{M}$ does not contain $\mathbf{P}$, then it has at most $O(n \log n)$ 1 's. In fact, $\mathbf{e x t}_{\text {mat }}(n, n, \mathbf{P})=\Theta(n \log n)$.

Tardos 236] proved that $\operatorname{ext}_{\text {mat }}(n, \mathbf{P})=n \log _{2} n+O(n)$.

Completing earlier works of Füredi and Péter Hajnal [116] Tardos [236] classified the ordered matrix Turán numbers for all small submatrices. The extremely slow growing inverse Ackermann function is denoted by $\alpha(n)$.

Theorem 12.7 ([116], 236]). If $\mathbf{P}$ is a 0-1 matrix with at most four 1 's, then

$$
\operatorname{ext}_{\text {mat }}(n, n, \mathbf{P})= \begin{cases}0 & \text { or } \\ \Theta(n), & \text { or } \\ \Theta(n \alpha(n)), & \text { or } \\ \Theta(n \log n), & \text { or } \\ \Theta\left(n^{3 / 2}\right) . & \end{cases}
$$

\subsection{Ordered matrices and the Stanley-Wilf conjecture on subpermutations}

Trying to prove the Erdős-Moser Conjecture, Füredi and Péter Hajnal [116] arrived at the following conjecture, proved by Marcus and Tardos.

Theorem 12.8 (Füredi-Hajnal conj. [116]/Marcus-Tardos theorem [187]). For all permutation matrices $\mathbf{P}$ we have $\mathbf{e x t}_{\text {mat }}(n, n, \mathbf{P})=O(n)$.

This time there was a famous Stanley-Wilf conjecture around, on the number of permutations "avoiding" a fixed permutation. To formulate it, we need to define the Permutation containment:

Definition 12.9 (Permutation containment). We say that a permutation $\sigma:[1, n] \rightarrow[1, n]$ contains a permutation $\pi:[1, k] \rightarrow[1, k]$, if there exist $1 \leq x_{1}<x_{2}<\cdots<x_{k} \leq n$ for which

$$
\sigma\left(x_{i}\right)<\sigma\left(x_{j}\right) \quad \text { if and only if } \quad \pi(i)<\pi(j) .
$$

The famous Stanley-Wilf conjecture 30 states that

Conjecture 12.10 (Stanley-Wilf). For any permutation pattern $q$, if $S_{n}(q)$ is the number of permutations of length $n$ avoiding the pattern $q$, then there is a constant $c_{q}$ so that $S_{n}(q) \leq c_{q}^{n}$.

\footnotetext{
${ }^{30}$ Marcus and Tardos [187 write that it is difficult to locate the corresponding reference.
} 
Theorem 12.11 (Klazar [158]). The Füredi-Hajnal conjecture implies the Stanley-Wilf conjecture.

So Marcus and G. Tardos settled this conjecture as well.

Remark 12.12. The permutation containment is just a subcase of the more general question. In some other cases there are definite differences between ordinary Turán type extremal problems and the ordered matrix problems. For a special matrix, where the corresponding graph is a tree, hence it has linear Turán function, our threshold function turns out to be $\Theta(n \log n)$.

\section{Applications in Geometry}

\subsection{Applicability of the Kövári-T. Sós-Turán bound}

We have mentioned that Theorem 2.22 is applicable in several cases. Here we mention only two.

(A) The Unit Distance Graph of the Plane contains no $K(2,3)$. Erdős used this to estimate the number of unit distances by $O\left(n^{3 / 2}\right)$.

(B) G. Megyesi and Endre Szabd31 answered a question of F. E. P. Hirzebruch using this theorem.

Assume that we are given $k$ smooth curves in the the Complex Projective Plane and assume that their union has only nodes and tacnodes 32 as singularities. Let $t(k)$ denote the maximum number of tacnodes in such cases. Hirzebruch proved that $t(k) \leq \frac{4}{9} k^{2}+\frac{4}{4} k$. Hirzebruch asked if $\lim \sup t(k) / k>0$.

Theorem 13.1 (G. Megyesi, and E. Szabó [191]). There exist three positive constants, $A, B$ and $C$ for which

$$
A k^{1+(B / \log \log k)} \leq t(k) \leq C k^{2-(1 / 7633)} .
$$

\subsection{Unit Distances}

Erdős was interested in the following problem:

Problem 13.2 (Unit distances). Given an $n$-element set in the $d$-dimensional Euclidean space $\mathbb{E}^{d}$, how many of the distances can be the same, say equal to 1 ?

\footnotetext{
${ }^{31}$ We use the longer versions of the names whenever we see chances to mix up authors of similar names.

${ }^{32}$ Tacnode means roughly that the curve is touching itself.
} 
Conjecture 13.3 (Unit distances). For any $\varepsilon>0$, there is an $n_{0}$ such that if $n>n_{0}$ and given an $n$-element set in the plane $\mathbb{E}^{2}$, then the number of unit distances is at most $n^{1+\varepsilon}$.

The motivation of this conjecture is - as Erdös observed - that if we arrange the $n=k \times k$ points into a $k \times k$ grid, and rescale this grid so that the "most popular" distance be 1 , then this distance will occur at most $n^{1+\varepsilon}$ times, (actually, approximately $n^{1+(c /(\log \log n))}$ times). So Erdös conjectured that the number of unit distances is in the plane has an upper bound of roughly this form.

The first upper bound was a trivial application of Theorem 2.22,

Theorem 13.4 (Unit distances, Erdös 1946). Given $n$ points in the plane, the number of unit distances among them is at most

$$
\operatorname{ex}\left(n, K_{2,3}\right)<\left(\frac{1}{\sqrt{2}}+o(1)\right) n^{3 / 2}
$$

In $\mathbb{E}^{3}$ the number of unit distances is at most

$$
\operatorname{ex}\left(n, K_{3,3}\right)<c_{3,3} n^{5 / 3}
$$

Proof. Since two circles intersect in at most 2 points, the Unit Distance Graph of $\mathbb{E}^{2}$ contains no $K_{2,3}$. This implies the first inequality. Since 3 unit balls intersect in at most 2 points, the Unit Distance Graph of $\mathbb{E}^{3}$ does not contain any $K_{3,3}$. This implies (13.1).

Remarks 13.5. (a) Everything is different for the higher dimensions: $\mathbb{E}^{4}$ contains two orthogonal circles of radii $\frac{1}{\sqrt{2}}$, and these form a $K(\infty, \infty)$ in the corresponding unit graphs of $\mathbb{E}^{d}$, for $d \geq 4$. (This is the so called Lenz Construction.) (See also Section 3.6.)

(b) How sharp is this application? As the reader can see, it is very far from the conjectured upper bound. However, just to improve it to $o\left(n^{3 / 2}\right)$ is non-trivial (Józsa-Szemerédi [152]). Actually, for the plane an $O\left(n^{4 / 3}\right)$ upper bound was proved by Beck and Spencer [20] and Spencer, Szemerédi and Trotter 233, which is sharp if we do not insist on Euclidean metric, only on "normed spaces". For this see the results of Peter Brass [35] and Pavel Valtr [245].

\subsection{Cells in line arrangements}

Let $\mathcal{I}(m, n)$ denote the maximum number of edges in $m$ distinct cells determined by an arrangement of $n$ lines in the plane. Canham [45] showed that 
for an absolute constant $c>0$

$$
\mathcal{I}(m, n)<c(m \sqrt{n}+n) .
$$

Indeed, if we construct a bipartite graph where one side of the vertex set consists of the $m$ cells (or any other family of $m$ convex sets with disjoint interiors), the other side of the vertex set consists of the $n$ (tangent) lines and two vertices are joined if the corresponding geometric objects are incident, then it is easy to see that this graph does not contain a $K_{5,2}$.

This was a first nontrivial step toward the determination of the exact order of the magnitude of $\mathcal{I}(m, n)$ by Clarkson, Edelsbrunner, Guibas, Sharir, and Welzl [49]; it is $\Theta\left(n^{2 / 3} m^{2 / 3}+n\right)$. More about this and other geometric applications see the monograph of Pach and Agarwal [199.

\section{Further connections and problems}

\subsection{Connections of hypergraphs and critical graphs}

We discussed Degenerate Hypergraph Extremal Problems in Section 10. Here we continue that line.

\section{Excluding the 3-uniform hypergraph cones}

Many of the other results, problems of [39] were also degenerate ones. One of them was where $\mathcal{T}$ is the family of triangulations of the 3-dimensional

sphere. This problem gave the name to this paper [39]. The crucial point was excluding the double cones:

Definition 14.1 ( $r$-cones). The vertices of the 3-uniform hypergraph $Q_{r, t}$ are $x_{1}, x_{2}, \ldots, x_{r}$, and $y_{1}, y_{2}, \ldots, y_{t}$ for some $t$, and the hyperedges are $x_{i} y_{j} y_{j+1}$, for all the possible $i, j$, where $y_{t+1}=y_{1}$. Further, $\mathbb{Q}_{r}:=\left\{Q_{r, t}: t=\right.$ $3,4,5, \ldots\}$.

Theorem 14.2 (Brown, Erdős, Sós, $r=2$, 39], Simonovits [219] $r \geq 2$ ).

$$
\operatorname{ex}\left(n, \mathbb{Q}_{r}\right):=O\left(n^{3-(1 / r)}\right)
$$

For $r=2,3$ there are matching lower bounds here. Actually, for $r=2$ Brown, Erdős and Sós gave a construction, where not only the double-cone was excluded, but all the triangulations of the sphere. In Simonovits' lower bound only the double cone was considered.

In 220. Simonovits returned to this question and - using the main idea of Brown's construction [36] - he proved 
Theorem 14.3 (Simonovits [220]). There are (finite geometric) 3-uniform hypergraphs without triple-cones (i.e. without hypergraphs from $\mathbb{Q}_{3}$ ) and still having at least $\mathrm{cn}^{3-(1 / 3)}$ triples.

We saw that for the family of triangulations of the sphere, and for the family of Double Cones the extremal number is $O\left(n^{3-(1 / 2)}\right)$ [39], (see [219]).

Brown, Erdős and T. Sós arrived at their question (most probably) since they wanted to generalize certain results from ordinary graphs to hypergraphs. Simonovits came from a completely different direction: he used this to disproved a conjecture of Gallai on independent vertices in 4-colourcritical graphs.

$G$ is colour-edge-critical, if deleting any edge of $G$, we get a $(\chi(G)-1)$ chromatic graph. The 3-colour-critical graphs are the odd cycles, so the problem of critical graphs becomes interesting for the 4-chromatic case. Here we shall restrict ourselves to this case and suggest the reader to read Bjarne Toft's results on this topic in general.

Erdös asked if a 4-colour-critical graph can have $c n^{2}$ edges and Bjarne Toft constructed such a 4-chromatic graph [240] of $\approx \frac{n^{2}}{16}$ edges. This and some related questions can also be found in Lovász' book: Combinatorial Exercises [176].

Gallai had many beautiful conjectures on 4-colour-critical graphs. One of them, however, was "completely demolished". He conjectured that if $G_{n}$ is 4-colour-critical, then $\alpha\left(G_{n}\right) \leq n / 2 . G_{4 m+2}=C_{2 m+1} \otimes C_{2 m+1}$ is 6-critical, with $d_{\min }\left(G_{4 m+2}\right)=2 m+3$. Simonovits - "blowing up" the vertices in one of the two odd cycles, - proved that there are 6-critical graphs $G_{n}$ with $\alpha\left(G_{n}\right)=n-o(n)$.

It turned out that slightly earlier Brown and Moon [38] already disproved Gallai's conjecture for the 4-chromatic case, with a "clever but simple" construction.

Theorem 14.4 (Brown and Moon [38). There exist 4-chromatic edgecritical graphs $G_{n}$ with $\alpha\left(G_{n}\right)>n-c \sqrt{n}$, for some constant $c>0$.

Next, Bjarne Toft came up with his construction, mentioned above. Using this and a hypergraph extremal theorem, Simonovits proved

Theorem 14.5. There exists a constant $c_{2}>0$ such that if $G_{n}$ is 4-colourcritical, then $\alpha\left(G_{n}\right) \leq n-c_{2} n^{2 / 5}$.

This was obtained as follows: Simonovits reduced the original problem to estimating the number of independent vertices of degree 3 in a 4-colourcritical graph. The neighborhoods of these vertices generated a 3-uniform 
hypergraph $\mathcal{H}_{m}^{(3)}$ on the remaining vertices. Simonovits - using the Sperner Lemma from Topology proved that if $I$ is a set of independent vertices of degree 3 , in $V\left(G_{n}\right)$, then for $m:=n-|I|,|I|<\mathbf{e x}_{3}\left(m, \mathbb{Q}_{2}\right)=O\left(m^{5 / 2}\right)$, see 219. He observed that $\mathcal{H}_{m}^{(3)}$ cannot contain double cones. This proved that $|I|<n-c n^{2 / 5}$.

(b) Lovász observed that instead of excluding the graphs from $\mathbb{Q}_{2}$ one can exclude a larger family, $\tilde{\mathbb{Q}}$ : those 3 -uniform hypergraphs which obey the conclusion of Sperner's lemma [175]: each pair $(x, y)$ is contained in an even number of hyperedges. This enabled him to completely settle this Gallai problem on colour-critical graphs. He proved that $\operatorname{ex}\left(n, \tilde{\mathbb{Q}}_{2}\right) \leq\left(\begin{array}{l}n \\ 2\end{array}\right)$. So he obtained $|I|<n-c \sqrt{n}$, in Gallai's problem. Besides proving and using a more applicable extremal graph theorem he also generalized the Brown-Moon construction.

(c) It was an interesting feature of Lovász' solution that to get an upper bound on $\operatorname{ex}(n, \tilde{\mathbb{Q}})$ he used linear algebra.

We finish this part by sketching the proof of Lovász on the upper bound.

Theorem 14.6 (Lovász [175]). Let $\mathbb{E}^{(3)}$ denote the family of 3-uniform hypergraphs $H$ in which each pair of vertices is contained in an even number of triplets (i.e. hyperedges). Then $\mathbf{e x}\left(n, \mathbb{E}^{(3)}\right) \leq\left(\begin{array}{l}n \\ 2\end{array}\right)$.

Proof (Sketch). Assume that $H_{n}^{(3)}$ contains no subgraphs from $\mathbb{E}^{(3)}$. Consider that vectorspace over $G F(2)$ of dimension $\left(\begin{array}{l}n \\ 2\end{array}\right)$ where the coordinates are indexed by pairs from $1, \ldots, n$. Represent each triple of $H_{n}^{(3)}$ by such a vector, where we have 1 in those coordinates which are pairs form our triple. The condition that $H_{n}^{(3)}$ contains no subgraphs from $\mathbb{E}^{(3)}$ translates into the fact, that these vectors are linearly independent. Hence their number is at most the dimension of the vector-space.

Now, repeating the original argument of Simonovits, Lovász obtained

Theorem 14.7. There exists a constant $c_{3}>0$ such that if $G_{n}$ is 4-colourcritical, then $\alpha\left(G_{n}\right) \leq n-c_{3} n^{1 / 2}$,

This with the Brown-Moon construction completely settles Gallai's original problem, providing a matching lower bound. Lovász proved a more general theorem, and extended the Brown-Moon construction as well. We close this part with a beautiful conjecture of Erdős:

Problem 14.8. Is it true that if $\left(G_{n}\right)$ is a sequence of 4-colour-critical graphs, then $d_{\min }\left(G_{n}\right)=o(n)$ ? 
(Simonovits 219] and Toft 240] succeeded in constructing 4-color-critical graphs with minimum degrees around $c \sqrt[3]{n}$.)

Further sources to read: Several related results can be found in Lovász [176].

\subsection{A multiplicative Sidon problem and $C_{2 k}$-free graphs}

As it was explained in Subsection 1.5, the Erdös problem about $\operatorname{ex}\left(n, C_{4}\right)$ in 60] was obtained from a multiplicative Sidon type question. He investigated subsets of integers of $A \subset\{1,2, \ldots, n\}$ with the property that for any four members of $A$ the pairwise products are distinct, $a_{i} a_{j} \neq a_{k} a_{\ell}$.

A. Sárközy, P. Erdős, and V. T. Sós [88] started investigating the more general problem.

Problem 14.9. Fix an integer $k$. How many integers can we take from $[1, n]$ if the product of no $k$ of them is a square.

Interestingly, this Problem also lead to Turán type questions, namely to $\operatorname{ex}\left(m, n, C_{2 k}\right)$ with $m \gg n$. Their conjecture (Conjecture 4.32 above) was proved by Győri [134, see Theorem 4.33. We shall not go into the number theoretic details; just refer the reader again to [134].

\subsection{Cycle-free subgraphs of the $d$-dimensional hypercube}

The $d$-dimensional hypercube, $Q^{d}$, is the graph whose vertex set is $\{0,1\}^{d}$ and whose edge set is the set of pairs that differ in exactly one coordinate, $e\left(Q^{d}\right)=d 2^{d-1}$. Let $\gamma\left(C_{\ell}\right)=\lim _{d \rightarrow \infty} \operatorname{ex}\left(Q^{d}, C_{4}\right) / e\left(Q^{d}\right)$. Note that $\gamma\left(C_{\ell}\right)$ exists, because $\operatorname{ex}\left(Q^{d}, C_{4}\right) / e\left(Q^{d}\right)$ is a non-increasing and bounded function of $d$. Considering the edges between the levels $2 i$ to $2 i+1$ one can see that $\operatorname{ex}\left(Q^{d}, C_{4}\right) \geq(1 / 2) e\left(Q^{d}\right)$. The following conjecture is still open.

Conjecture 14.10 (Erdős [74]). $\operatorname{ex}\left(Q^{d}, C_{4}\right)=\left(\frac{1}{2}+o(1)\right) e\left(Q^{d}\right)$.

The best upper bound $\gamma\left(C_{4}\right) \leq 0.6226$ was obtained by Thomason and Wagner [238, slightly improving the result of Chung [46].

Erdős [74] also asked whether $\operatorname{ex}\left(Q^{d}, C_{2 k}\right)$ is $o(d) 2^{d}$ for $k>2$. This was answered negatively for $C_{6}$ by Chung [46, showing that $\gamma\left(C_{6}\right) \geq 1 / 4$. The best known results for $C_{6}$ are $1 / 3 \leq \gamma\left(C_{6}\right)<0.3941$ due to Conder [50] and $\mathrm{Lu}$ 180, respectively.

On the other hand, for every $t \geq 2$ the inequalities

$$
\operatorname{ex}\left(Q^{d}, C_{4 t}\right) \leq O\left(d^{\frac{1}{2}-\frac{1}{2 t}} 2^{d}\right) \quad \text { and } \quad \operatorname{ex}\left(Q^{d}, C_{4 t+6}\right)=O\left(d^{\frac{15}{16}-\frac{1}{16 t}} 2^{d}\right)
$$


were proved by Chung [46 and Füredi and Özkahya [118, respectively. Hence $\gamma\left(C_{2 k}\right)=0$, except $\gamma\left(C_{4}\right) \geq 1 / 2, \gamma\left(C_{6}\right) \geq 1 / 3$ and the problem of deciding wether $\gamma\left(C_{10}\right)=0$ is still open.

Conlon [51] generalized (14.1) by showing $\operatorname{ex}\left(Q^{d}, H\right)=o\left(e\left(Q^{d}\right)\right)$ for all $H$ that admit a $k$-partite representation, also satisfied by each $H=C_{2 k}$ except for $k \in\{2,3,5\}$.

\subsection{Two problems of Erdős}

Of course, we should close with two open problem of Erdős. The first one is the general version of that problem which was solved in [124] and [141, see Section 1.1.

Conjecture 14.11 (Erdös 75$]$ ). Suppose that $G$ is a graph on $(2 k+1) n$ vertices and of odd girth $2 k+1$. Then $G$ contains at most $n^{2 k+1}$ induced cycles of length $2 k+1$.

The next conjecture is also very famous and is motivated by the blown up pentagon (if we restrict it to $k=2$.)

Conjecture 14.12 (Erdős [75]). Suppose that $G$ is a graph on $(2 k+1) n$ vertices and of odd girth at least $2 k+1$. Then $G$ can be made bipartite by omitting at most $n^{2}$ edges.

For the best known results here, for $k=1$, see Erdös, Faudree, Pach, and Spencer [77] and Erdős, Györi, and Simonovits [81].

\section{Acknowledgements}

The authors are greatly indebted for fruitful discussions and helps to a great number of colleagues, among others to R. Faudree, E. Győri, and Z. Nagy.

\section{References}

[1] M. Ajtai, J. Komlós, M. Simonovits, and E. Szemerédi: On the approximative solution of the Erdős-Sós conjecture on trees, (manuscript).

[2] M. Ajtai, J. Komlós, M. Simonovits, and E. Szemerédi: Some elementary lemmas on the Erdős-T. Sós conjecture for trees, (manuscript).

[3] M. Ajtai, J. Komlós, M. Simonovits, and E. Szemerédi: The solution of the Erdős-Sós conjecture for large trees, (manuscript, in preparation).

[4] M. Ajtai, J. Komlós, and E. Szemerédi: On a conjecture of Loebl, in Graph theory, Combinatorics, and Algorithms, Vol. 1, 2 (Kalamazoo, MI, 1992), Wiley-Intersci. Publ., pp. 1135-1146. Wiley, New York, 1995. 
[5] P. Allen, P. Keevash, B. Sudakov, and J. Verstraëte: Turán numbers of bipartite graphs plus an odd cycle, submitted.

[6] N. Alon: Eigenvalues and expanders, Combinatorica 6 (1983), 83-96.

[7] N. Alon: Tools from higher algebra, in : "Handbook of Combinatorics", R. L. Graham, M. Grötschel and L. Lovász, eds, North Holland (1995), Chapter 32, pp. 1749-1783.

[8] N. Alon, S. Hoory, and N. Linial: The Moore bound for irregular graphs, Graphs Combin. 18 (2002), no. 1, 53-57.

[9] N. Alon, M. Krivelevich, and B. Sudakov: Turán numbers of bipartite graphs and related Ramsey-type questions, Combin. Probab. Comput. 12 (2003), no. 5-6, 477-494.

[10] N. Alon and V. D. Milman: $\lambda_{1}$-isoperimetric inequalities for graphs and superconcentrators, J. Combin. Theory Ser. B 38 (1985), 73-88.

[11] N. Alon, L. Rónyai, and T. Szabó: Norm-graphs: variations and applications, J. Combin. Theory Ser. B 76 (1999), 280-290.

[12] L. Babai and B. Guiduli: Spectral extrema for graphs: the Zarankiewicz problem, Electronic J. Combin. 15 (2009), R123.

[13] R. Baer: Polarities in finite projective planes, Bull. Amer. Math. Soc. 52 (1946), 77-93.

[14] C. Balbuena, P. García-Vázquez, X. Marcote, and J. C. Valenzuela: New results on the Zarankiewicz problem, Discrete Math. 307 (2007), no. 17-18, $2322-2327$.

[15] C. Balbuena, P. García-Vázquez, X. Marcote, and J. C. Valenzuela: Counterexample to a conjecture of Györi on $C_{2 l}$-free bipartite graphs, Discrete Math. 307 (2007), no. 6, 748-749.

[16] C. Balbuena, P. García-Vázquez, X. Marcote, and J. C. Valenzuela: Extremal K(s,t)-free bipartite graphs, Discrete Math. Theor. Comput. Sci. 10 (2008), no. $3,35-48$.

[17] P. N. Balister, B. Bollobás, O. M. Riordan, and R. H. Schelp: Graphs with large maximum degree containing no odd cycles of a given length, J. Combin. Theory B 87 (2003), 366-373.

[18] P. N. Balister, E. Győri, J. Lehel, and R. H. Schelp: Connected graphs without long paths, Discrete Math 308 (2008), no. 19, 4487-4494.

[19] S. Ball and V. Pepe: Asymptotic improvements to the lower bound of certain bipartite Turán numbers, Combin. Probab. Comput. 21 (2012), no. 3, 323329.

[20] J. Beck and J. Spencer: Unit distances, J. Combin. Theory Ser. A 37 (1984), 231-238.

[21] F. Behrend: On sets of integers which contain no three terms in arithmetic progression, Proc. Nat. Acad. Sci. US. 32 (1956), 331-332.

[22] C. T. Benson: Minimal regular graphs of girths eight and twelve, Canad. J. Math. 18 (1966), 1091-1094.

[23] D. Bienstock and E. Györi: An extremal problem on sparse 0-1 matrices, SIAM J. Discrete Math. 4 (1991), no. 1, 17-27.

[24] P. Blagojević, B. Bukh, and R. Karasev: Turán numbers for $K_{s, t}$-free graphs: 
Füredi-Simonovits: Degenerate (bipartite) extremal graph problems

topological obstructions and algebraic constructions, arXiv:1108.5254v3, 3 Jun 2012.

[25] B. Bollobás: Cycles modulo k, Bull. London Math. Soc. 9 (1977), no. 1, 97-98.

[26] B. Bollobás: Extremal Graph Theory, Academic Press, London, 1978.

[27] B. Bollobás: Random Graphs, Academic Press, London, 1985.

[28] B. Bollobás: Extremal graph theory, in: R. L. Graham, M. Grötschel, and L. Lovász (Eds.), Handbook of Combinatorics, Elsevier Science, Amsterdam, 1995, pp. 1231-1292.

[29] B. Bollobás and A. Thomason: Proof of a conjecture of Mader, Erdös and Hajnal on topological subgraphs, European J. Combin 19 (1998), 883-887.

[30] J. A. Bondy: Basic graph theory: paths and circuits, Handbook of Combinatorics, Vol. I., pp. 3-110, Elsevier, Amsterdam, 1995.

[31] J. A. Bondy: Extremal problems of Paul Erdős on circuits in graphs, Paul Erdös and his mathematics, II (Budapest, 1999), 135-156, Bolyai Soc. Math. Stud., 11, János Bolyai Math. Soc., Budapest, 2002.

[32] J. A. Bondy and M. Simonovits: Cycles or even length in graphs, J. Combin. Theory Ser. B 16 (1974), 97-105.

[33] J. A. Bondy and A. Vince: Cycles in a graph whose lengths differ by one or two, J. Graph Theory 27 (1998), 11-15.

[34] S. Brandt and E. Dobson: The Erdős-Sós conjecture for graphs of girth 5, Selected papers in honour of Paul Erdős on the occasion of his 80th birthday (Keszthely, 1993), Discrete Math. 150 (1996), no. 1-3. 411-414.

[35] P. Brass: Erdős distance problems in normed spaces, Comput. Geom. 6 (1996), no. 4, 195-214.

[36] W. G. Brown: On graphs that do not contain a Thomsen graph, Canad. Math. Bull. 9 (1966), 281-285.

[37] W. G. Brown: On the non-existence of a type of regular graphs of girth 5, Canad. J. Math. 19 (1967), 644-648.

[38] W. G. Brown and J. W. Moon: Sur les ensembles de sommets indépendants dans les graphes chromatiques minimaux, (French), Canad. J. Math. 21 (1969), 274-278.

[39] W. G. Brown, P. Erdős and V. T. Sós: On the existence of triangulated spheres in 3-graphs, and related problems, Period Math. Hungar. 3 (1973), $221-228$.

[40] W. G. Brown, P. Erdős and V. T. Sós: Some extremal problems on $r$-graphs, New Directions in the Theory of Graphs (ed. F. Harary), Academic Press, New York, 1973, pp. 53-63.

[41] W. G. Brown and M. Simonovits: Digraph extremal problems, hypergraph extremal problems, and the densities of graph structures, Discrete Math. 48 (1984), no. 2-3, 147-162.

[42] W. G. Brown, and M. Simonovits: Extremal multigraph and digraph problems, Paul Erdős and his mathematics, II (Budapest, 1999), pp. 157-203, Bolyai Soc. Math. Stud., 11, János Bolyai Math. Soc., Budapest, 2002.

[43] L. Caccetta and K. Vijayan: Long cycles in subgraphs with prescribed min- 
imum degree, Discrete Math. 97 (1991), no. 1-3, 69-81.

[44] D. de Caen and L. A. Székely: The maximum size of 4- and 6-cycle free bipartite graphs on $m, n$ Vertices, Sets, Graphs and Numbers (Budapest, 1991), Colloquium Mathematical Society János Bolyai, vol. 60, North-Holland, Amsterdam, 1992, pp. 135-142.

[45] R. Canham: A theorem on arrangements of lines in the plane, Israel J. Math. 7 (1969), 393-397.

[46] F. Chung: Subgraphs of a hypercube containing no small even cycles, J. Graph Theory 16 (1992), 273-286.

[47] F. R. K. Chung and R. L. Graham: Erdős on Graphs: His Legacy of Unsolved Problems, A. K. Peters Ltd., Wellesley, MA, 1998.

[48] C. R. J. Clapham, A. Flockart, and J. Sheehan: Graphs without four-cycles, J. Graph Theory 13 (1989), 29-47.

[49] K. Clarkson, H. Edelsbrunner, L. J. Guibas, M. Sharir, and E. Welzl: Combinatorial complexity bounds for arrangements of curves and spheres, Discrete Comput. Geom. 5 (1990), no. 2, 99-160.

[50] M. Conder: Hexagon-free subgraphs of hypercubes, J. Graph Theory 17 (1993), 477-479.

[51] D. Conlon: An extremal theorem in the hypercube, Electron. J. Combin. 17 (2010), Research Paper 111.

[52] D. Conlon, J. Fox, and B. Sudakov: An approximate version of Sidorenko's conjecture, Geom. Funct. Anal. 20 (2010), no. 6, 1354-1366.

[53] D. Conlon, J. Fox, and B. Sudakov: Sidorenko's conjecture for a class of graphs: an exposition, http://arxiv.org/abs/1209.0184

[54] O. Cooley: Proof of the Loebl-Komlós-Sós conjecture for large, dense graphs, Discrete Math. 309 (2009), no. 21, 6190-6228.

[55] K. Čulík: Teilweise Lösung eines verallgemeinerten Problems von K. Zarankiewicz, Ann. Polon. Math. 3 (1956), 165-168.

[56] D. M. Cvetkovič, M. Doob, and H. Sachs: Spectra of Graphs, Academic Press Inc., New York, 1980.

[57] G. Damásdi, T. Héger, and T. Szőnyi: The Zarankiewicz problem, cages, and geometries, manuscript 2013.

[58] H. Edelsbrunner and P. Hajnal: A lower bound on the number of unit distances between the vertices of a convex polygon, J. Combin. Theory Ser. A 56 (1991), no. 2, 312-316.

[59] Paul Erdős: Erdős homepage (his scanned in papers up to 1989): www.renyi.hu/ p_erdos.

[60] P. Erdős: On sequences of integers no one of which divides the product of two others, and some related problems, Mitt. Forschungsinst. Math. u. Mech. Tomsk 2 (1938), 74-82.

[61] P. Erdős: Graph theory and probability I, Canad. J. Math. 11 (1959), 34-38.

[62] P. Erdős: Graph theory and probability II, Canad. J. Math. 13 (1961), 346352.

[63] P. Erdős: Extremal problems in graph theory, Proc. Sympos. Smolenice, 1963, pp. 29-36, Publ. House Czechoslovak Acad. Sci., Prague, 1964. 
[64] P. Erdős: Some applications of probability to graph theory and combinatorial problems, Theory of Graphs and its Applications (Proc. Sympos. Smolenice, 1963), pp. 133-136, Publ. House Czech. Acad. Sci., Prague, 1964.

[65] P. Erdős: On some extremal problems in graph theory, Israel J. Math. 3 (1965), 113-116.

[66] P. Erdős: Some recent results on extremal problems in graph theory, Theory of Graphs (ed P. Rosenstiehl), (Internat. Sympos., Rome, 1966), Gordon and Breach, New York, and Dunod, Paris, 1967, pp. 117-123.

[67] P. Erdős: On some new inequalities concerning extremal properties of graphs, Theory of Graphs (P. Erdős and G. Katona, Eds.), Academic Press, Nev. York, 1968, pp. 77-81.

[68] P. Erdős: The Art of Counting (ed. J. Spencer), The MIT Press, Cambridge, Mass., 1973.

[69] P. Erdős: Problems and results on finite and infinite combinatorial analysis, in Infinite and Finite Sets (Proc. Conf., Keszthely, Hungary, 1973), pp. 403424, Proc. Colloq. Math. Soc. J. Bolyai 10, Bolyai-North-Holland, 1975.

[70] P. Erdős: Some recent progress on extremal problems in graph theory, Congr. Numerantium 14 (1975), 3-14.

[71] P. Erdős: Problems and results in combinatorial analysis, Colloquio Internazionale sulle Teorie Combinatorie (Rome, 1973), Tomo II, Atti dei Convegni Lincei, No. 17, pp. 3-17, Accad. Naz. Lincei, Rome, 1976.

[72] P. Erdős: Problems and results in graph theory and combinatorial analysis, Graph theory and related topics (Proc. Conf., Univ. Waterloo, Waterloo, Ont., 1977), pp. 153-163, Academic Press, New York-London.

[73] P. Erdős: On the combinatorial problems which I would most like to see solved, Combinatorica 1 (1981), no. 1, 25-42.

[74] P. Erdős: On some problems in graph theory, combinatorial analysis and combinatorial number theory, Graph Theory and Combinatorics (Cambridge, 1983), pp. 1-17, Academic Press, London, 1984.

[75] P. Erdős: Two problems in extremal graph theory. Graphs Combin. 2 (1986), no. 1, 189-190.

[76] P. Erdős: On some of my favourite theorems, Combinatorics, Paul Erdős is eighty, Vol. 2 (Keszthely, 1993), 97-132, Bolyai Soc. Math. Stud., 2, János Bolyai Math. Soc., Budapest, 1996.

[77] P. Erdős, R. J. Faudree, J. Pach, and J. Spencer: How to make a graph bipartite, J. Combin. Theory Ser. B 45 (1988), no. 1, 86-98.

[78] P. Erdős, R. J. Faudree, R. H. Schelp, and M. Simonovits: An extremal result for paths, Graph theory and its applications: East and West (Jinan, 1986), 155-162, Ann. New York Acad. Sci., 576, New York Acad. Sci., New York, 1989.

[79] P. Erdős, Z. Füredi, M. Loebl, and V. T. Sós: Discrepancy of trees, Studia Sci. Math. Hungar. 30 (995), no. 1-2, 47-57.

[80] P. Erdös and T. Gallai: On maximal paths and circuits of graphs, Acta Math. Acad. Sci. Hungar. 10 (1959), 337-356.

[81] P. Erdős, E. Győri, and M. Simonovits: How many edges should be deleted to 
make a triangle-free graph bipartite? Sets, graphs and numbers (Budapest, 1991), pp. 239-263, Colloq. Math. Soc. János Bolyai, 60, North-Holland, Amsterdam, 1992.

[82] P. Erdős, G. Harcos, and J. Pach: Popular distances in 3-space, Discrete Math. 200 (1999), no. 1-3, 95-99.

[83] P. Erdős and L. Moser: Problem 11, Canad. Math. Bull. 2 (1959), 43.

[84] P. Erdős and A. Rényi: On the evolution of random graphs, Magyar Tud. Akad. Mat. Kutató Int. Közl. 5 (1960), 17-61.

[85] P. Erdős and A. Rényi: On a problem in the theory of graphs, Magyar Tud. Akad. Mat. Kutató Int. Közl. 7 (1962), 623-641.

[86] P. Erdős, A. Rényi, and Vera T. Sós: On a problem of graph theory, Stud Sci. Math. Hung. 1 (1966), 215-235.

[87] P. Erdős and H. Sachs: Reguläre Graphen gegebener Taillenweite mit minimaler Knotenzahl (in German), Wiss. Z. Martin-Luther-Univ. HalleWittenberg Math.-Natur. Reihe 12 (1963), 251-257.

[88] P. Erdős, A. Sárközy, and V. T. Sós: On product representation of powers, I, European J. Combin. 16 (1995), 567-588.

[89] P. Erdős and M. Simonovits: A limit theorem in graph theory, Studia Sci. Math. Hungar. 1 (1966), 51-57.

[90] P. Erdős and M. Simonovits: Some extremal problems in graph theory, Combinatorial Theory and Its Applications, I. (Proc. Colloq. Balatonfüred, 1969), North Holland, Amsterdam, 1970, pp. 377-390.

[91] P. Erdös and M. Simonovits: An extremal graph problem, Acta Math. Acad. Sci. Hungar. 22 (1971/72), 275-282.

[92] P. Erdős and M. Simonovits: Cube-supersaturated graphs and related problems, Progress in Graph Theory (Waterloo, Ont., 1982), pp. 203-218, Academic Press, Toronto, Ont., 1984.

[93] P. Erdős and M. Simonovits: Compactness results in extremal graph theory, Combinatorica 2 (1982), no. 3, 275-288.

[94] P. Erdős and M. Simonovits: Supersaturated graphs and hypergraphs, Combinatorica 3 (1983), 181-192.

[95] P. Erdös and A. M. Stone: On the structure of linear graphs, Bull. Amer. Math. Soc 52 (1946), 1087-1091.

[96] G. Fan: Distribution of cycle lengths in graphs, J. Combin. Theory Ser. B 84 (2002), 187-202.

[97] G. Fan, Xuezheng Lv, and Pei Wang: Cycles in 2-connected graphs, J. Combin. Theory Ser. B 92 (2004), no. 2, 379-394.

[98] R. J. Faudree and R. H. Schelp: Path Ramsey numbers in multicolorings, J. Combin. Theory Ser. B 19 (1975), no. 2, 150-160.

[99] R. J. Faudree and M. Simonovits: On a class of degenerate extremal graph problems, Combinatorica 3 (1983), 83-93.

[100] R. J. Faudree and M. Simonovits: On a class of degenerate extremal problems II, preprint.

[101] J. Fox and B. Sudakov: Dependent random choice, Random Structures Algorithms 38 (2011), no. 1-2, 68-99. 
[102] F. A. Firke, P. M. Kosek, E. D. Nash, and J. Williford: Extremal graphs without 4-cycles, http://arxiv.org/pdf/1201.4912v1.pdf

[103] Z. Füredi: Graphs without quadrilaterals, J. Combin. Theory Ser. B 34 (1983), 187-190.

[104] Z. Füredi: Quadrilateral-free graphs with maximum number of edges, preprint 1988 , http://www.math.uiuc.edu/ ${ }^{2}$-furedi/PUBS/furedi_C4from1988.pdf

[105] Z. Füredi: Graphs of diameter 3 with the minimum number of edges, Graphs Combin. 6 (1990), no. 4, 333-337.

[106] Z. Füredi: The maximum number of unit distances in a convex $n$-gon, J. Combin. Theory Ser. A 55 (1990), no. 2, 316-320.

[107] Z. Füredi: On a Turán type problem of Erdős, Combinatorica 11 (1991), $75-79$.

[108] Z. Füredi: Turán type problems, in Surveys in Combinatorics, London Math. Soc. Lecture Note Ser. 166, Cambridge University Press, Cambridge, UK, 1991, pp. 253-300.

[109] Z. Füredi: The maximum number of edges in a minimal graph of diameter 2, J. Graph Theory 16 (1992), no. 1, 81-98.

[110] Z. Füredi: Extremal hypergraphs and combinatorial geometry, Proceedings of the International Congress of Mathematicians, Vol. 1, 2 (Zürich, 1994), pp. 1343-1352, Birkhäuser, Basel, 1995.

[111] Z. Füredi: On the number of edges of quadrilateral-free graphs, J. Combin. Theory Ser. B 68 (1996), 1-6.

[112] Z. Füredi: An upper bound on Zarankiewicz problem, Combin. Probab. Comput. 5 (1996), no. 1, 29-33.

[113] Z. Füredi: New asymptotics for bipartite Turán numbers, J. Combin. Theory Ser. A 75 (1996), no. 1, 141-144.

[114] Z. Füredi: On the number of fivecycles, manuscript, unpublished, superseded by 124

[115] Z. Füredi: On a theorem of Erdős and Simonovits on graphs not containing the cube, to appear

[116] Z. Füredi and Peter Hajnal: Davenport-Schinzel theory of matrices, Discrete Math. 103 (1992), 231-251.

[117] Z. Füredi, A. Naor, and J. Verstraëte: On the Turán number for the hexagon, Adv. Math. 203 (2006), no. 2, 476-496.

[118] Z. Füredi and L. Özkahya: On even-cycle-free subgraphs of the hypercube, J. Combin. Theory Ser. A 118 (2011), 1816-1819.

[119] Z. Füredi, O. Pikhurko, and M. Simonovits: The Turán density of the hypergraph $\{a b c, a d e, b d e, c d e\}$, Electronic J. Combin. 10 (2003), R18.

[120] Z. Füredi and M. Simonovits: Triple systems not containing a Fano configuration, Combin. Probab. Comput. 14 (2005), no. 4, 467-484.

[121] Z. Füredi and D. West: Ramsey theory and bandwidth of graphs, Graphs and Combin. 17 (2001), 463-471.

[122] D. K. Garnick, Y. H. H. Kwong, and F. Lazebnik: Extremal graphs without three-cycles or four-cycles, J. Graph Theory 17 (1993), no. 5, 633-645. 
[123] D. K. Garnick, and N. A. Nieuwejaar, Non-isomorphic extremal graphs without three-cycles and four-cycles, J. Combin. Math. Combin. Comput. 12 (1992), 33-56.

[124] A. Grzesik: On the maximum number of five-cycles in a triangle-free graph, J. Combin. Theory Ser. B 102 (2012), no. 5, 1061-1066.

[125] J. R. Griggs and Chih-Chang Ho: On the half-half case of the Zarankiewicz problem, Discrete Math. 249 (2002), no. 1-3, 95-104.

[126] J. Griggs, J. Ouyang: (0,1)-matrices with no half-half submatrix of ones, European J. Combin. 18 (1997), 751-761.

[127] J. R. Griggs, M. Simonovits, and George Rubin Thomas: Extremal graphs with bounded densities of small subgraphs, J. Graph Theory 29 (1998), no. 3, 185-207.

[128] R. K. Guy: A problem of Zarankiewicz, in: Theory of Graphs (Proc. Colloq., Tihany, 1966), pp. 119-150. Academic Press, New York 1968.

[129] R. K. Guy and S. Znám: A problem of Zarankiewicz, Recent Progress in Combinatorics (Proc. Third Waterloo Conf. on Combinatorics, 1968), pp. 237-243. Academic Press, New York 1969.

[130] A. Gyárfás: Graphs with k odd cycle lengths, Discrete Math. 103 (1992), 41-48.

[131] A. Gyárfás, J. Komlós, and E. Szemerédi: On the distribution of cycle lengths in graphs, J. Graph Theory 8 (1984), 441-462.

[132] A. Gyárfás, C. C. Rousseau, and R. H. Schelp: An extremal problem for paths in bipartite graphs, J. Graph Theory 8 (1984), 83-95.

[133] E. Győri: On the number of $C_{5}$ 's in a triangle-free graph, Combinatorica 9 (1989), 101-102.

[134] E. Győri: $C_{6}$-free bipartite graphs and product representation of squares, Graphs Combin. (Marseille, 1995), Discrete Math. 165/166 (1997), 371-375.

[135] E. Győri: Triangle-free hypergraphs, Combin. Prob. Comput. 15 (2006), 185191.

[136] E. Győri, B. Rothschild, and A. Ruciński: Every graph is contained in a sparsest possible balanced graph, Math. Proc. Cambridge Philos. Soc. 98 (1985), no. 3, 397-401.

[137] R. Häggkvist and A. D. Scott: Arithmetic progressions of cycles, Tech. Rep. Mat. Inst. Umeä Univ. 16, (1998).

[138] R. Häggkvist and A. Scott: Cycles of nearly equal length in cubic graphs, Preprint.

[139] S. Hartman, J. Mycielski, C. Ryll-Nardzevski: Systèmes spéciaux de points à coordonnées entiéres, Colloq. Math. 3 (1954), 84-85, (Bericht Über di Tagung der Poln Math Gesellschaft, Wroclaw, am 20. September 1951.)

[140] H. Hatami: Graph norms and Sidorenko's conjecture, Israel J. Math. 175 (2010), 125-150.

[141] H. Hatami, J. Hladký, D. Král, S. Norine, and A. Razborov: On the number of pentagons in triangle-free graphs, J. Combin. Theory Ser. A 120 (2013), no. 3, 722-732.

[142] H. Hatami and S. Norine: Undecidability of linear inequalities in graph ho- 
momorphism densities, J. Amer. Math. Soc. 24 (2011), no. 2, 547-565.

[143] J. Hladký, J. Komlós, D. Piguet, M. Simonovits, M. Stein, and E. Szemerédi: The approximate Loebl-Komlós-Sós Conjecture, submitted, on arXiv:1211.3050.v1, 2012, Nov 13.

[144] J. Hladký and D. Piguet: Loebl-Komlós-Sós Conjecture: dense case, Manuscript (arXiv:0805:4834).

[145] M. N. Huxley and H. Iwaniec: Bombieri's theorem in short intervals, Mathematika 22 (1975), 188-194.

[146] C. Hyltén-Cavallius: On a combinatorial problem, Colloq. Math. 6 (1958), 59-65.

[147] W. Imrich: Explicit construction of graphs without small cycles, Combinatorica 4 (1984), 53-59.

[148] C. Jagger, P. St́ovíček, and A. Thomason: Multiplicities of subgraphs, Combinatorica 16 (1996), no. 1, 123-141.

[149] S. Janson, T. Łuczak, and A. Ruciński: Random Graphs, Wiley-Interscience Series in Discrete Mathematics and Optimization. Wiley-Interscience, New York, 2000. xii+333 pp.

[150] T. Jiang: Compact topological minors in graphs, J. Graph Theory 67 (2011), $139-152$.

[151] T. Jiang and R. Seiver: Turán numbers of subdivided graphs, SIAM J. Discrete Math. 26 (2012), no. 3, 1238-1255.

[152] S. Józsa and E. Szemerédi: The number of unit distance on the plane, Infinite and finite sets (Colloq., Keszthely, 1973; dedicated to P. Erdős on his 60th birthday), Vol. II, pp. 939-950. Colloq. Math. Soc. János Bolyai, Vol. 10, North-Holland, Amsterdam, 1975.

[153] G. O. H. Katona: Turán's graph theorem and probability theory, Turán Memorial: Number theory, Analysis and Combinatorics, de Gruyter, Berlin, to appear.

[154] Gy. Katona, T. Nemetz, and M. Simonovits: On a problem of Turán in the theory of graphs, Mat. Lapok 15 (1964), 228-238.

[155] P. Keevash: Hypergraph Turan problems, Surveys in Combinatorics, Cambridge University Press, 2011, 83-140.

[156] P. Keevash and B. Sudakov: The Turan number of the Fano plane, Combinatorica 25 (2005), 561-574.

[157] P. Keevash, B. Sudakov, and J. Verstraëte: On a conjecture of Erdős and Simonovits: even cycles, Combinatorica, to appear.

[158] M. Klazar: The Füredi-Hajnal conjecture implies the Stanley-Wilf conjecture, in: D. Krob, A. A. Mikhalev, A. V. Mikhalev (Eds.), Formal Power Series and Algebraic Combinatorics, Springer, Berlin, 2000, pp. 250-255.

[159] J. Kollár, L. Rónyai, and T. Szabó: Norm graphs and bipartite Turán numbers, Combinatorica 16 (1996), 399-406.

[160] J. Komlós and E. Szemerédi: Topological cliques in graphs, Combin. Probab. Comput. 3 (1994), no. 2, 247-256.

[161] J. Komlós and E. Szemerédi: Topological cliques in graphs II, Combin. Probab. Comput. 5 (1996), 79-90. 
Füredi-Simonovits: Degenerate (bipartite) extremal graph problems

[162] G. N. Kopylov: Maximal paths and cycles in a graph, Dokl. Akad. Nauk SSSR 234 (1977), no. 1, 19-21. (English translation: Soviet Math. Dokl. 18 (1977), no. 3, 593-596.)

[163] A. Kostochka and L. Pyber: Small topological complete subgraphs of "dense" graphs, Combinatorica 8 (1988), 83-86.

[164] T. Kövári, V. T. Sós, and P. Turán: On a problem of K. Zarankiewicz, Colloq. Math. 3 (1954), 50-57.

[165] D. Kühn and D. Osthus: Four-cycles in graphs without a given even cycle, J. Graph Theory 48 (2005), 147-156.

[166] T. Lam and J. Verstraëte: A note on graphs without short even cycles, Electron. J. Combin. 12 (2005), Note 5, 6 pp.

[167] F. Lazebnik and D. Mubayi: New lower bounds for Ramsey numbers of graphs and hypergraphs, Adv. in Appl. Math. 28 (2002), no. 3-4, 544-559.

[168] F. Lazebnik and V. A. Ustimenko, New examples of graphs without small cycles and of large size, European J. Combin. 14 (1993), no.5, 445-460.

[169] F. Lazebnik, V. A. Ustimenko, and A. J. Woldar: Properties of certain families of $2 k$-cycle-free graphs, J. Combin. Theory Ser. B 60 (1994), no. 2, 293-298.

[170] F. Lazebnik, V. A. Ustimenko, and A. J. Woldar: A new series of dense graphs of high girth, Bull. Amer. Math. Soc. 32 (1995), no. 1, 73-79.

[171] F. Lazebnik, V. A. Ustimenko, and A. J. Woldar, Polarities and $2 k$-cycle-free graphs, Discrete Math. 197/198 (1999), 503-513.

[172] F. Lazebnik and A. J. Woldar: General properties of some families of graphs defined by systems of equations, J. Graph Theory 38 (2001), no. 2, 65-86.

[173] J. Lenz and D. Mubayi: Multicolor Ramsey numbers for complete bipartite versus complete graphs, arXiv 1201.2123, 26 pp.

[174] B. Lidický, Hong Liu, and C. Palmer: On the Turán number of forests, arXiv 1204.3102 .

[175] L. Lovász: Independent sets in critical chromatic graphs, Studia Sci. Math. Hungar. 8 (1973), 165-168.

[176] L. Lovász: Combinatorial Problems and Exercises, 2nd Ed., North-Holland, Amsterdam, 1993.

[177] L. Lovász: Large Networks and Graph Limits, Colloquium Publications 2012, 475 pp.

[178] L. Lovász and M. Simonovits: On the number of complete subgraphs of a graph II, Studies in Pure Mathematics, pp. 459-495, (dedicated to the memory of P. Turán), Akadémiai Kiadó and Birkhäuser Verlag 1982.

[179] A. A. Razborov: Flag algebras, J. Symbolic Logic 72 (2007), no. 4, 12391282.

[180] Linyuan Lu: Hexagon-free subgraphs in hypercube $Q_{n}$, private communication.

[181] A. Lubotzky, R. Phillips, and P. Sarnak: Ramanujan graphs, Combinatorica 8 (1988), no. 3, 261-277.

[182] A. McLennan: The Erdős-Sós conjecture for trees of diameter four, J. Graph Theory 49 (2005), no. 4, 291-301. 
[183] W. Mader: Homomorphieeigenschaften und mittlere Kantendichte von Graphen, Math. Ann. 174 (1967), 265-268.

[184] W. Mader: Topological subgraphs in graphs of large girth, Combinatorica 18 (1998), no. 3, 405-412.

[185] W. Mader: Topological minors in graphs of minimum degree $n$, Contemporary trends in discrete mathematics (Štiřín Castle, 1997), 199-211, DIMACS Ser. Discrete Math. Theoret. Comput. Sci., 49, Amer. Math. Soc., Providence, RI, 1999.

[186] W. Mader: Graphs with $3 n-6$ edges not containing a subdivision of $K_{5}$, Combinatorica 25 (2005), no. 4, 425-438.

[187] A. Marcus and G. Tardos: Excluded permutation matrices and the StanleyWilf conjecture, J. Combin. Theory Ser. A 107 (2004), no. 1, 153-160.

[188] G. A. Margulis: Explicit construction of graphs without short cycles and low density codes, Combinatorica 2 (1982), 71-78.

[189] G. A. Margulis: Arithmetic groups and graphs without short cycles, in: 6th Int. Symp. on Information Theory, Tashkent, Abstracts 1, 1984, pp. 123-125 (in Russian).

[190] G. A. Margulis: Explicit group-theoretical construction of combinatorial schemes and their application to the design of expanders and concentrators, J. Problems of Inform. Trans. 24 (1988), 39-46; translation from Problemy Peredachi Informatsii 24 (January-March 1988), 51-60.

[191] G. Megyesi and E. Szabó: On the tacnodes of configurations of conics in the projective plane, Math. Ann. 305 (1996), no. 4, 693-703.

[192] M. Molloy and B. Reed: Graph Colouring and the Probabilistic Method, Algorithms and Combinatorics, 23. Springer-Verlag, Berlin, 2002, xiv +326 pp.

[193] B. Montágh: Unavoidable substructures, PHD Thesis, University of Memphis, May 2005.

[194] M. Mörs: A new result on the problem of Zarankiewicz, J. Combin. Theory Ser. A 31 (1981), no. 2, 126-130.

[195] D. Mubayi and Gy. Turán: Finding bipartite subgraphs efficiently, Inform. Process. Lett. 110 (2010), no. 5, 174-177.

[196] Z. L. Nagy: A multipartite version of the Turán problem — density conditions and eigenvalues, Electron. J. Combin. 18 (2011), no. 1, Paper 46, 15 pp.

[197] V. Nikiforov: Bounds on graph eigenvalues II, Linear Algebra Appl. 427 (2007), 183-189.

[198] V. Nikiforov: A contribution to the Zarankiewicz problem, Linear Algebra Appl. 432 (2010), no. 6, 1405-1411.

[199] J. Pach and P. K. Agarwal: Combinatorial Geometry, Wiley-Interscience, New York, 1995. xiv+354 pp.

[200] P. P. Pálfy and M. Szalay, in: Turán Memorial: Number theory, Analysis and Combinatorics, de Gruyter, Berlin, to appear.

[201] D. Piguet and M. J. Stein: Loebl-Komlós-Sós conjecture for trees of diameter 5, Electron. J. Combin. 15 (2008), Research Paper 106, 11 pp. 
[202] D. Piguet and M. J. Stein: An approximate version of the Loebl-Komlós-Sós conjecture, J. Combin. Theory Ser. B 102 (2012), no. 1, 102-125.

[203] O. Pikhurko: A note on the Turán function of even cycles, Proc. Amer. Math Soc. 140 (2012), 3687-3992.

[204] R. Pinchasi and M. Sharir: On graphs that do not contain the cube and related problems, Combinatorica 25 (2005), no. 5, 615-623.

[205] C. Reiher: The clique density theorem, arxiv1212.2454.

[206] I. Reiman: Über ein Problem von K. Zarankiewicz, Acta Math. Acad. Sci. Hungar. 9 (1958), no. 3-4, 269-273.

[207] I. Reiman: An extremal problem in graph theory, (Hungarian). Mat. Lapok 12 (1961), 44-53.

[208] A. Rényi: Selected Papers of Alfréd Rényi, Akadémiai Kiadó, 1976 (ed. Paul Turán).

[209] V. Rödl and M. Schacht: Extremal results for random graphs, in this volume.

[210] I. Z. Ruzsa and E. Szemerédi: Triple systems with no six points carrying three triangles, Combinatorics (Proc. Fifth Hungarian Colloq., Keszthely, 1976), Vol. II, pp. 939-945, Colloq. Math. Soc. János Bolyai, 18, North-Holland, Amsterdam-New York, 1978.

[211] J.-F. Saclè and M. Woźniak: A note on the Erdős-Sós conjecture for graphs without $C_{4}$, J. Combin. Theory Ser. B 70 (1997), no.2, 367-372.

[212] G. N. Sárközy: Cycles in bipartite graphs and an application in number theory, J. Graph Theory 19 (1995), 323-331.

[213] A. Scott: Szemerédi's regularity lemma for matrices and sparse graphs, Combin. Probab. Comput. 20 (2011), no. 3, 455-466.

[214] Jian Shen: On two Turán numbers, J. Graph Theory 51 (2006), 244-250.

[215] A. F. Sidorenko: Asymptotic solution for a new class of forbidden $r$-graphs, Combinatorica 9 (1989), no. 2, 207-215.

[216] A. Sidorenko: A correlation inequality for bipartite graphs, Graphs Combin. 9 (1993), no. 2, 201-204.

[217] A. F. Sidorenko: What do we know and what we do not know about Turán Numbers, Graphs Combin. 11 (1995), no. 2, 179-199.

[218] M. Simonovits: A method for solving extremal problems in graph theory, Theory of Graphs, Proc. Colloq. Tihany, (1966), (P. Erdős and G. Katona, Eds.), pp. 279-319, Acad. Press, New York, 1968.

[219] M. Simonovits: On colour-critical graphs, Studia Sci. Math. Hungar. 7 (1972), 67-81.

[220] M. Simonovits: Note on a hypergraph extremal problem, Hypergraph Seminar, Columbus Ohio USA, 1972, (C. Berge and D. K. Ray-Chaudhuri, Eds.), Lecture Notes in Mathematics 411, pp. 147-151, Springer Verlag, 1974.

[221] M. Simonovits: Extremal graph problems with symmetrical extremal graphs, additional chromatic conditions, Discrete Math. 7 (1974), 349-376.

[222] M. Simonovits: On Paul Turán's influence on graph theory, J. Graph Theory 1 (1977), no. 2, 102-116.

[223] M. Simonovits: Extremal graph problems and graph products, Studies in Pure Mathematics, pp. 669-680, (dedicated to the memory of P. Turán), 
Akadémiai Kiadó and Birkhäuser Verlag 1982.

[224] M. Simonovits: Extremal graph theory, in: L. W. Beineke, R. J. Wilson (Eds.), Selected Topics in Graph Theory II., pp. 161-200, Academic Press, London, 1983.

[225] M. Simonovits: Extremal graph problems, degenerate extremal problems and supersaturated graphs, Progress in graph Theory, (Bondy and Murty, Eds.), pp. 419-438, Academic Press, 1984.

[226] M. Simonovits: How to solve a Turán type extremal graph problem? (linear decomposition), Contemporary trends in discrete mathematics (Stirin Castle, 1997), pp. 283-305, DIMACS Ser. Discrete Math. Theoret. Comput. Sci., 49, Amer. Math. Soc., Providence, RI, 1999.

[227] M. Simonovits: Paul Erdős' influence on extremal graph theory, The mathematics of Paul Erdős, II., pp. 148-192, Algorithms Combin., 14, Springer, Berlin, 1997.

[228] M. Simonovits: Paul Erdős' influence on extremal graph theory, The new version of the old paper [227.

[229] M. Simonovits: Paul Turán's influence in Combinatorics, in Turán Memorial: Number Theory, Analysis, and Combinations, De Gruyter, to appear.

[230] M. Simonovits and V. T. Sós: Ramsey-Turán theory, Combinatorics, graph theory, algorithms and applications, Discrete Math. 229 (2001), no. 1-3, 293340 .

[231] R. R. Singleton: On minimal graphs of maximum even girth, J. Combinatorial Theory 1 (1966), 306-332.

[232] V. T. Sós: Remarks on the connection of graph theory, finite geometry and block designs, Colloquio Internazionale sulle Teorie Combinatorie (Roma, 1973), Tomo II, pp. 223-233, Atti dei Convegni Lincei, No. 17, Accad. Naz. Lincei, Rome, 1976.

[233] J. Spencer, E. Szemerédi, and W. T. Trotter: Unit distances in the Euclidean plane, Graph theory and combinatorics (Cambridge, 1983), pp. 293-303, Academic Press, London, 1984.

[234] B. Sudakov and J. Verstraëte: Cycle lengths in sparse graphs, Combinatorica 28 (2008), no. 3, 357-372.

[235] E. Szemerédi: Regular partitions of graphs, Problemes Combinatoires et Theorie des Graphes (ed. I.-C. Bermond et al.), pp. 399-401, CNRS, Paris, 1978.

[236] G. Tardos: On 0-1 matrices and small excluded submatrices, J. Combin. Th. Ser. A 111 (2005), 266-288.

[237] A. G. Thomason: A disproof of a conjecture of Erdős in Ramsey Theory, J. London Math. Soc. 39 (1989), 246-255.

[238] A. Thomason and P. Wagner: Bounding the size of square-free subgraphs of the hypercube, Discrete Math. 309 (2009), 1730-1735.

[239] C. M. Timmons: Ordered Turán Problems, Lecture no. 1086-05-1067 on the Joint Mathematics Meetings, San Diego, CA, January 9, 2013.

[240] B. Toft: Two theorems on critical 4-chromatic graphs, Studia Sci. Math. Hungar. 7 (1972), 83-89. 
[241] P. Turán: On a theorem of Hardy-Ramanujan, Journal of London Math Soc. 9 (1934), 274-276.

[242] P. Turán: On an extremal problem in graph theory, (Hungarian), Mat. Fiz. Lapok 48 (1941), 436-452.

[243] P. Turán: On the theory of graphs, Colloq. Math. 3 (1954), 19-30.

[244] P. Turán: A note of welcome, J. Graph Theory 1 (1977), 7-9.

[245] P. Valtr: Strictly convex norms allowing many unit distances and related touching questions, manuscript.

[246] J. Verstraëte: On arithmetic progressions of cycle lengths in graphs, Combin. Probab. Comput. 9 (2000), no.4, 369-373.

[247] R. Wenger: Extremal graphs with no $C_{4}$ 's, $C_{6}$ 's, or $C_{10}$ 's, J. Combin. Theory Ser. B 52 (1991), no. 1, 113-116.

[248] R. M. Wilson: An existence theory for pairwise balanced designs, III. Proof of the existence conjectures, J. Combin. Theory Ser. A 18 (1975), 71-79.

[249] D. R. Woodall: Maximal circuits of graphs I, Acta Math. Acad. Sci. Hungar. 28 (1976), no. 1-2, 77-80.

[250] D. R. Woodall: Maximal circuits of graphs II, Studia Sci. Math. Hungar. 10 (1975), no. 1-2, 103-109.

[251] M. Woźniak: On the Erdős-Sós conjecture, J. Graph Theory, 21 (1996), no. 2, 229-234.

[252] Y. Yuansheng and P. Rowlinson: On extremal graphs without four-cycles, Utilitas Math. 41 (1992), 204-210.

[253] Y. Yuansheng and P. Rowlinson: On graphs without 6-cycles and related Ramsey numbers, Utilitas Math. 44 (1993), 192-196.

[254] K. Zarankiewicz: Problem 101, Colloquium Mathematicum 2 (1951), p. 301.

[255] Yi Zhao: Proof of the $(n / 2-n / 2-n / 2)$ conjecture for large $n$, Electron. J. Combin. 18 (2011), Paper 27.

[256] Š. Znám: On a combinatorical problem of K. Zarankiewicz, Colloq. Math. 11 (1963), 81-84.

[257] Š. Znám: Two improvements of a result concerning a problem of K. Zarankiewicz, Colloq. Math. 13 (1964/1965), 255-258. 